\title{
Modellierung des turbulenten Austausches zwischen der Prandtl-Schicht und der Waldatmosphäre
}

\author{
Dissertation \\ zur Erlangung des Doktorgrades \\ der mathematisch-naturwissenschaftlichen Fakultäten \\ der Georg-August-Universität \\ zu Göttingen
}

vorgelegt von:

Erik Nützmann

aus:

Schönebeck/Elbe

Göttingen, April 1999 
D7

Referent:

Koreferent:

Tag der mündlichen Prüfung: 27.04.1999

Prof. Dr. H. Eckelmann

Prof. Dr. D. Ronneberger 


\section{Inhaltsverzeichnis}

1 Einleitung 4

1.1 Wald und atmosphärische Turbulenz . . . . . . . . . . . . 4

1.2 Aufbau der atmosphärischen Grenzschicht . . . . . . . . . 8

2 Lokale Turbulenzschließung: K-Theorie 12

2.1 Das Schließungsproblem . . . . . . . . . . . . . . . . . . . 12

2.2 K-Theorie als lokales Schließungsverfahren . . . . . . . . . . . 14

3 Transiliente Turbulenztheorie $\quad 17$

3.1 Einführung . . . . . . . . . . . . . . . . . . 17

3.2 Das Mischungspotential . . . . . . . . . . . . . . . 21

3.2.1 Konvektive Grenzschicht ．. . . . . . . . . . . . . 21

3.2.2 Turbulente kinetische Energie . . . . . . . . . . . . . 22

3.2.3 Parametrisierung des Mischungspotentials auf der Basis der Richardsonzahl . . . . . . . . . . . . . . . 26

3.2.4 Parametrisierung des Mischungspotentials auf der Basis der Turbulenzenergie . . . . . . . . . . . 27

3.3 Abhängigkeit der Mischungsmatrix vom Modellzeitschritt und Massennormierung . . . . . . . . . . . . 35

3.4 Übergang zur Transilienzmatrix . . . . . . . . . . . . . . . . . . . . . . . . .

3.5 Struktur der Transilienzmatrix _. . . . . . . . . . . . . 40

3.6 Berechnung der turbulenten Flüsse . . . . . . . . . . . . 43

3.7 Mischungsintensität, Prozeßspektrum und Mischungsweglänge 44

3.8 Verwendung des transilienten Schließungsansatzes in einem Grenzschichtmodell . . . . . . . . . . . . . . . . . 46

4 Der Einfluß hoher Pflanzenbestände $\quad 48$

4.1 Formwiderstand . . . . . . . . . . . . . . . . . 48

4.2 Nachlaufturbulenz . . . . . . . . . . . . . . . . 55

4.3 Der Einfluß auf vertikale turbulente Umlagerungen . . . . . . 56 
5 Das Modell $\quad 58$

5.1 Aufbau des Atmosphären - und Bestandsmodells . . . . . . . . 58

5.2 Modellparameter und Randwerte . . . . . . . . . . . . . . . . . 59

5.3 Bestimmung der Erdoberflächentemperatur . . . . . . . . . . . . 64

5.4 Lösung der Wärmeleitungsgleichung im Boden . . . . . . . . . 67

5.5 Bodenfeuchtemodellierung . . . . . . . . . . . 68

5.6 Berechnung der Strahlungsflüsse im Bestand . . . . . . . . . . 70

5.7 Energieaustausch zwischen der Vegetation und der Bestandsluft 74

5.8 Berechnung der Temperatur der Vegetationsoberfläche . . . . . 77

5.9 Berechnung des Nadelwasserpotentials . . . . . . . . . 78

5.10 Behandlung der Niederschläge . . . . . . . . . . . . 80

5.11 Festlegung der Parameter der transilienten Schliessung . . . . 82

6 Validierung des Modells $\quad 83$

6.1 Auswahl des zu modellierenden Zeitraumes . . . . . . . . . . 83

6.1.1 Parametrisierung der Nachlaufturbulenz . . . . . . . . . 84

6.2 Strahlungsmodellierung . . . . . . . . . . . . . . . 84

6.3 Bodentemperaturmodellierung . . . . . . . . . . . . . . . . . 85

6.4 Bodenfeuchtemodellierung . . . . . . . . . . . 86

6.5 Stationäre Modellrechnungen . . . . . . . . . . . . . . . . 86

6.6 Instationäre Modellrechnungen . . . . . . . . . . . . . . . . . 91

$\begin{array}{lll}7 & \text { Zusammenfassung } & 104\end{array}$ 


\section{Kapitel 1}

\section{Einleitung}

\subsection{Wald und atmosphärische Turbulenz}

Im Rahmen der Klima - und Ökosystemforschung hat die Vorhersage von Quellen und Senken atmosphärischer Spurenstoffe eine besondere Bedeutung. Hintergrund hierfür ist in der Klimaforschung die Berechnung der weiteren Entwicklung der Treibhausgaskonzentrationen im Rahmen von Klimavorhersagemodellen, in der Ökosystemforschung die Abschätzung der Auswirkungen von Spurenstoffen auf die Entwicklung von Ökosystemen. Besondere Bedeutung für beide Forschungsbereiche hat die Untersuchung von Waldökosystemen, die auch heute noch einen großen Teil (ca. $25 \%$ ) der Landoberfläche der Erde einnehmen [13]. Dabei hat sich herausgestellt, daß Wälder sehr empfindlich auf Umweltveränderungen reagieren und dabei auch als effektive Quelle von Treibhausgasen (insbesondere $\mathrm{CO}_{2}$ ) auftreten können. Die Lokalisierung und quantitative Erfassung von natürlichen bzw. durch anthropogene Einwirkung auf die Natur hervorgerufenen Quellen ${ }^{1}$ und Senken von Treibhausgasen erfordert aber wegen der geringen atmosphärischen Konzentrationen eine sehr genaue Beschreibung des atmosphärischen Austausches mit den Ökosystemen. Der Kenntnis der vertikalen Verteilung atmosphärischer Spurenstoffe innerhalb der Waldbestände kommt dabei eine besondere Bedeutung zu. Aus diesem Grunde wurde im Rahmen der Ökosystemforschung eine Vielzahl von Transportmodellen für das System Boden-Vegetation-Atmosphäre (Soil Vegetation Atmosphere Transfer Models - SVAT's) entwickelt ([14], [46], [35], [26], [18], [29] ). Die zur Zeit am häufigsten verwendeten Modelle des turbulenten atmosphärischen Austausches innerhalb von Waldbeständen benutzen einfache Widerstandsformulierungen oder Gradientansätze, bei denen von einer Analogie zwischen Diffusion und atmosphärischer Turbulenz ausgegangen wird (K-Theorie), die aber streng genommen nicht gegeben ist. Diese Modelle folgen der Annahme, der Austausch erfolge nur zwischen benachbarten Schichten entgegen dem lokalen Konzentrationsgradienten. Der eigentlich irreführende Begriff "turbulente Diffusion“ weist darauf hin. Aus Vergleichen mit Messungen ist jedoch bekannt, daß ein solcher Diffusionsansatz die atmo-

${ }^{1}$ nicht zu verwechseln mit den direkten anthropogenen Emissionen 
sphärischen Verhältnisse nur unzureichend beschreibt, da die Atmosphäre als häufig turbulentes Medium ein ganzes Spektrum von Wirbelgrößen enthält, die auch den direkten Austausch zwischen weiter entfernten Schichten bewirken können. Dies ist der Grund, weshalb die genannten Ansätze oft nur unzureichende Ergebnisse liefern. So werden insbesondere in der atmosphärischen Grenzschicht und innerhalb von Vegetationsbeständen Flüsse gegen den lokalen Gradienten (counter gradient fluxes) beobachtet, welche diese einfachen Modelle nicht beschreiben können. Um zur Lösung dieses Problems beizutragen, wurden u.a. mehrdimensionale Modelle entwickelt, die die einzelnen Wirbel explizit auflösen. Solche Modelle liefern zwar sehr realistische Ergebnisse, sind jedoch aufgrund ihrer Komplexität insbesondere für Klimamodelle viel zu aufwendig. Auch Gradientansätze mit Termen höherer Ordnung sind hier numerisch zu aufwendig, da für die Diskretisierung der Ableitungen höherer Ordnung nach der Vertikalkoordinate unter Umständen sehr eng benachbarte Modellschichten notwendig werden, womit sich der Rechenaufwand für das gesamte Klimamodell drastisch erhöhen kann. Einige SVAT-Modelle haben zudem den Nachteil, daß sie schon kurz über dem Bestand ihre Obergrenze erreichen und somit die atmosphärische Grenzschicht bzw. deren Effekte auf den Bestand nicht vollständig simulieren können [26].

Neuere Modelle simulieren die atmosphärische Wirkung hoher Pflanzenbestände und den turbulenten Austausch in der gesamten atmosphärischen Grenzschicht (sogenannte gekoppelte Grenzschichtmodelle). Dabei wird meist ebenfalls auf den Diffusionsansatz zurückgegriffen ([46], [26]). Insbesondere im Falle einer atmosphärischen Grenzschicht mit Waldbestand werden jedoch Phänomene, wie Flüsse gegen den lokalen Gradienten (counter gradient fluxes), beobachtet, die auf das gleichzeitige Zusammenwirken von Wirbeln unterschiedlicher Größenordnung zurückgeführt werden, also mit einem einfachen Diffusionsansatz nicht erfaßt werden können. Solche Phänomene können mit den oben genannten Grenzschichtmodellen nicht erfaßt werden. Dieses Problem kann durch Schließungsansätze gelöst werden, die nicht mehr auf dem Diffusionsansatz beruhen, dabei aber die einzelnen Wirbel nicht explizit auflösen müssen, so daß die aufzuwendende Zeit für die Durchführung von Modellexperimenten in vertretbarem Rahmen bleibt. Ein solcher Ansatz beruht z.B. auf der Transilienten Turbulenzschließung. Dabei wird die von Stull [41] ursprünglich für eine Atmosphäre ohne Vegetation entwickelte Transiliente Turbulenzschließung in modifizierter Form für die Simulation des turbulenten Austausches in und über einem Waldbestand angepaßt. Solche Modelle liegen von Inclán et al. 1996 [18] und Ni 1996 [28] in unterschiedlichen Ausführungen vor. Zwei Effekte spielen eine zentrale Rolle bei der Beschreibung der atmosphärischen Zustandsgrößen in Beständen hoher Vegetation. Das ist zum einen das Auftreten eines sekundären Maximums der Windgeschwindigkeit im Stammraum und zum anderen das Auftreten von Eigenschaftsflüssen gegen die lokalen Eigenschaftsgradienten („,counter-gradient" Flüsse). Der letzte Effekt tritt nicht nur beim vertikalen Impulstransport, sondern auch bei den Flüssen sensibler und latenter Wärme im Bestand auf. Für das Auftreten eines sekundären Windmaximums werden 2 Ursachen diskutiert:

- Das seitliche Einströmen in den Bestand an Waldrändern. Durch den höheren aerodynamischen Widerstand im Kronenraum gegenüber dem Stammraum 


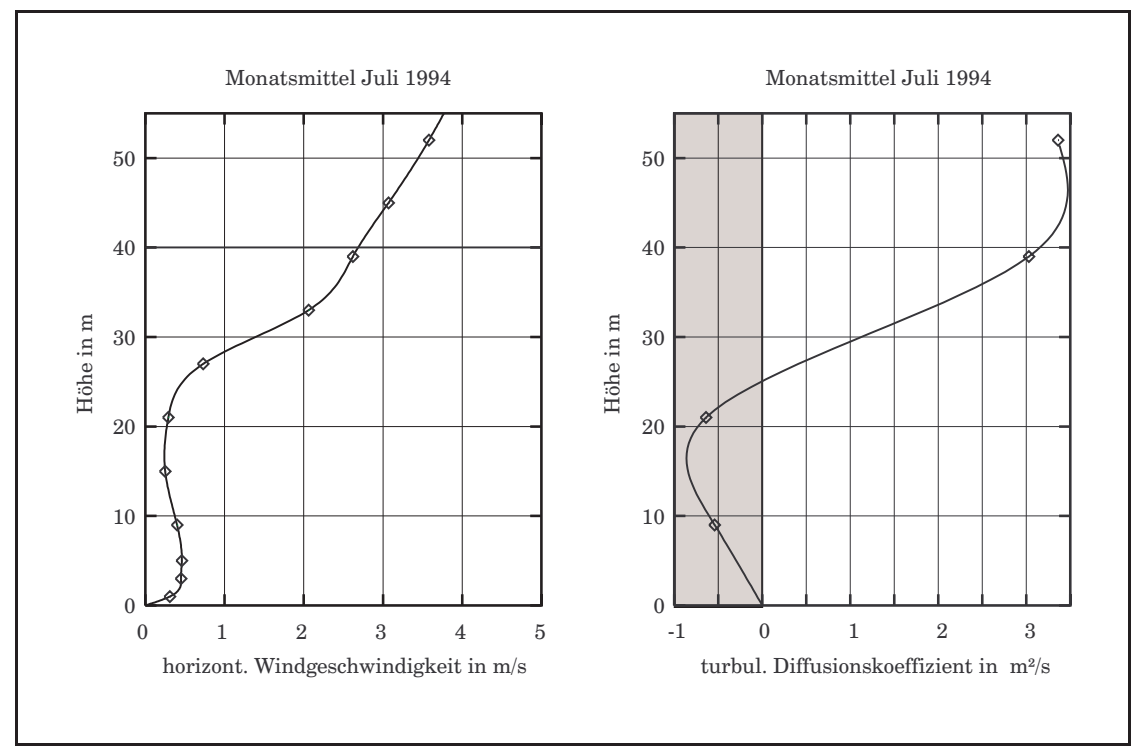

Abbildung 1.1: Mittleres Profil der horizontalen Windgeschwindigkeit und für ausgewählte Höhen berechnete Diffusionskoeffizienten für Impuls in einem Fichtenbestand im Solling für den Juli 1994

wird die Strömung im Kronenraum stärker abgebremst und es kann zur Ausbildung eines sekundären Windmaximums kommen, wenn ein deutlich ausgebildeter Stammraum vorhanden ist. Andere Effekte, wie thermische Schichtung im Bestand und Stärke des Windes spielen dabei auch eine Rolle.

- Der vertikale turbulente Transport von Impuls aus Schichten oberhalb des Bestandes (Prandtl-Schicht der Atmosphäre) in den Stammraum läßt sich zu einem großen Teil auf großskalige Wirbel (Large-Eddies) mit Durchmessern von der Größenordnung der Bestandshöhe und größer zurückführen. Während kleinere Wirbel im Kronenraum stark abgebremst werden und evtl. dissipieren, können die Large-Eddies aufgrund ihrer Trägheit bis in den Stammraum vordringen, und Impuls aus der Prandtl-Schicht und damit auch die dortigen relativ hohen Windgeschwindigkeiten direkt in den Stammraum transferieren. Dieser zweite Punkt liefert auch eine Erklärung für die ",counter-gradient" Flüsse der atmosphärischen Größen.

Einen Eindruck vom auftretenden sekundären Windmaximum im Stammraum vermittelt das Profil der Monatsmittelwerte der horizontalen Windgeschwindigkeit in einem Fichtenbestand im Solling vom Juli 1994, wie es in Abb. 1.1 (links) dargestellt ist. Auf der rechten Seite der Abbildung sind die für 4 Höhen berechneten mittleren turbulenten Diffusionskoeffizienten für Impuls mit einem hindurchgelegten angenommenen Profil für denselben Monat dargestellt. Am Boden ist anzunehmen, daß die atmosphärische Turbulenz verschwindet und somit auch der turbulente Diffusionskoeffizient. Deshalb geht die durch die Punkte gelegte Kurve am Erdboden gegen Null. Der grau unterlegte Bereich hebt die negativen Werte des Diffusionskoeffizienten im Stamm- und un- 
teren Kronenraum hervor.

Die Bezeichnung „,turbulenter Diffusionskoeffizient" erinnert an die angenommene Analogie zwischen dem Austausch durch molekulare Diffusion und Turbulenz. Es gibt jedoch nur wenige Argumente, die diese Analogie unterstützen. Eines der Hauptargumente entspringt wohl eigentlich mehr dem zu Zeiten des Beginns der Turbulenzforschung bestehenden Zwang bzw. Wunsch nach einer einfachen Möglichkeit der Beschreibung turbulenter Transporte. Wie bei der Diffusion wurde oder wird meist auch heute noch angenommen, der turbulente Transport einer Eigenschaft erfolge parallel zum physikalischen Gradienten dieser Eigenschaft, und sei diesem entgegengesetzt gerichtet. Danach wird also der turbulente Fluß als proportional dem negativen physikalischen Gradienten angesehen, wobei die Analogie zur molekularen Diffusion nur gilt, wenn der Proportionalitätsfaktor positiv ist. Nur wenn dies der Fall ist, werden durch die turbulenten Flüsse Konzentrationsgradienten abgebaut, wie es auch bei der molekularen Diffusion beobachtet wird.

So wäre also auch bei den aus Messungen abgeleiteten turbulenten Diffusionkoeffizienten zu erwarten gewesen, daß diese in allen Höhen positiv sind. Dies ist jedoch nicht der Fall. Diesen Werten entsprechen somit turbulente Impulsflüsse in Richtung des physikalischen vertikalen Gradienten des Impulses. Unglücklicherweise werden in der Meteorologie die Gradienten immer vom Ort des höheren Wertes einer Eigenschaft zum Ort des niederen Wertes einer Eigenschaft definiert, so daß man in diesem Fall von einem Fluß entgegen dem meteorologischen Gradienten (engl. counter gradient flux) spricht.

Bei dem hier betrachteten Fichtenbestand im Solling scheinen beide Punkte eine Rolle zu spielen. Stellt man die Differenzen der Windgeschwindigkeit zwischen Kronen- und Stammraum in Abhängigkeit von der Windrichtung über dem Bestand dar (siehe Abb. 1.2), so findet man, daß ein sekundäres Windmaximum (Windgeschwindigkeit im Stammraum ist größer als die im Kronenraum) unabhängig von der Windrichtung immer auftreten kann. Eine Häufung bei Wind aus nordwestlicher Richtung erklärt sich aus der in dieser Richtung liegenden Lichtung, was einen horizontalen Windgeschwindigkeitsgradienten zwischen der Lichtung und dem Stammraum zur Folge hat, und damit durch Impulsadvektion in den Stammraum. Es ist also anzunehmen, daß beide Effekte beim Zustandekommen des sekundären Windmaximums eine Rolle spielen.

Die thermische Schichtung über dem Bestand scheint dagegen keinen Einfluß auf das Auftreten eines sekundären Windmaximums zu haben (siehe Abb. 1.3).

Für eine genauere Untersuchung der turbulenten Stoffflüsse in und über einem Waldbestand ist die Kenntnis des Aufbaus der atmosphärischen Grenzschicht im allgemeinen und der atmosphärischen Grenzschicht über Waldbeständen im besonderen notwendig. Auf diesen Punkt soll deshalb im Rahmen einer Einleitung zu dieser Arbeit im folgenden Abschnitt eingegangen werden. 


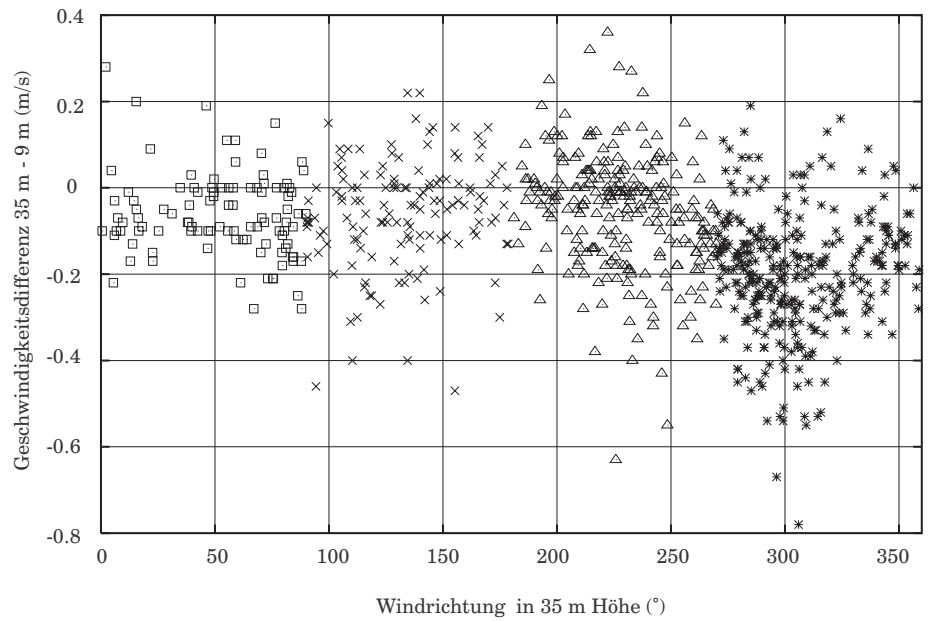

Abbildung 1.2: Abhängigkeit der Differenz der Windgeschwindigkeit zwischen Kronen- und Stammraum in einem Fichtenbestand im Solling von der Windrichtung über dem Bestand (Halbstundenmittel 1997)

\subsection{Aufbau der atmosphärischen Grenzschicht}

Der untere Teil der Atmosphäre im Höhenbereich von bis 8-12 km, in dem sich das Wettergeschehen abspielt, wird als Troposphäre bezeichnet. Sie wird nach oben durch die Tropopause begrenzt. Die mittlere Höhe der Tropopause hängt von der geographischen Breite und der Jahreszeit ab, ist aber auch täglichen Schwankungen unterworfen, so daß man keinen einheitlichen Wert der Tropopausenhöhe für die gesamte Erde angeben kann. Für eine mittlere geographische Breite könnte man im Jahresmittel eine Höhe von etwa 10 km angeben [38].

Die untere Begrenzung der Troposphäre zur festen Erde bildet die atmosphärische Grenzschicht. Diese Grenzschicht ist der Bereich der Atmosphäre, in dem sich in den Profilen der atmosphärischen Eigenschaften und Flüsse der Einfluß der Erdoberfläche unmittelbar (d.h. in einem zeitlichen Rahmen von Stunden) bemerkbar wird. Im Unterschied zu den in der technischen Hydrodynamik anzutreffenden Grenzschichten, weist die atmosphärische Grenzschicht insgesamt im allgemeinen keine Dickenzunahme in Strömungsrichtung auf, da die Hauptursache für die Grenzschichtentstehung, die Bodenreibung, überall gegeben ist. Eine solche Dickenzunahme tritt in der Meteorologie nur an internen Grenzschichten auf, die sich bilden, wenn sich die Eigenschaften der Unterlage sprunghaft ändern, z.B. beim Überströmen eines Waldrandes.

Insbesondere kann bei thermisch instabiler Schichtung, wie sie durch die tageszeitliche Erwärmung der Erdoberfläche auftreten kann, aufgrund der Rauhigkeit der Oberfläche und der dadurch verursachten mechanischen Reibung eine intensive turbulente Durchmischung hervorgerufen werden. Je nach der vorherrschenden Rauhigkeit, der Temperaturschichtung und der Windgeschwindigkeit kann die Höhe der atmosphärische Grenzschicht unterschiedlich ausfallen. Als mittlerer Wert werden häufig 1000 m angesetzt. Dabei ist insbeson- 


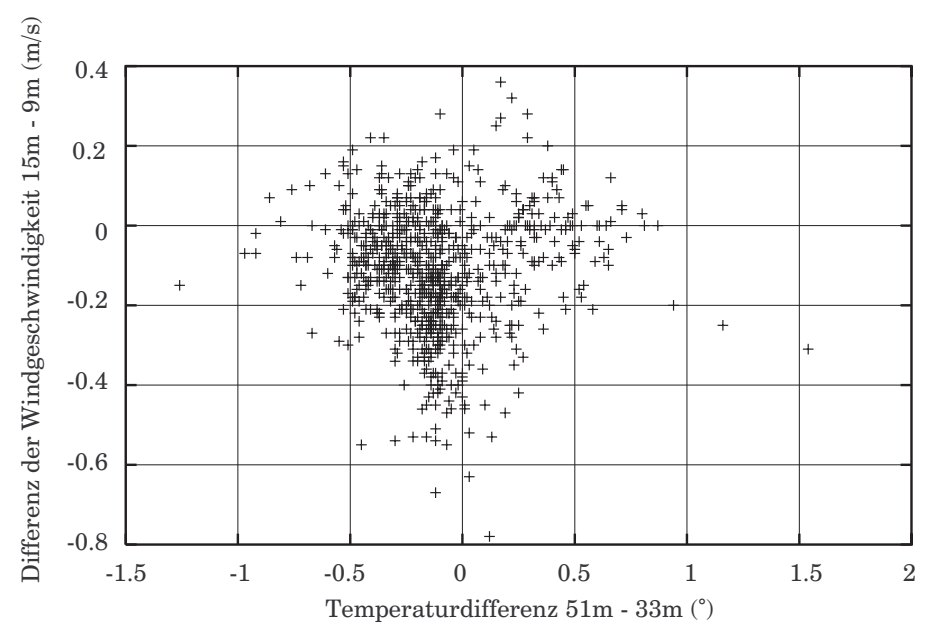

Abbildung 1.3: Abhängigkeit der Differenz der Windgeschwindigkeit zwischen Kronen- und Stammraum in einem Fichtenbestand im Solling von der thermischen Schichtung über dem Bestand (Halbstundenmittel 1997)

dere der Tagesgang der Grenzschichthöhe wichtig, so daß sich im Tagesverlauf eine Höhenvariation von einigen 100 Metern bis zu einigen 1000 Metern ergeben kann [38].

Die atmosphärische Grenzschicht läßt sich anhand der in verschiedenen Höhen vorherrschenden physikalischen Prozesse weiter unterteilen. Die für die Teilschichten angegebenen Dicken beziehen sich in der Literatur oft auf die mittleren Verhältnisse über den Ozeanen, da diese mehr als 70 \% der Erdoberfläche bedecken [34], können jedoch als grösenordnungsmäsige Abschätzung der Verhältnisse über einer Festlandsoberfläche ohne hohem Vegetationsbestand herangezogen werden.

Direkt an die Erdoberfläche schließt sich eine molekulare Grenzschicht an, deren Dicke nur im Millimeter-Bereich liegt. Diese Schicht wird oft als zähe Unterschicht bezeichnet. Hier finden nur diffusive molekulare Transportprozesse statt. Daran schließt sich die dynamische Unterschicht an, in der sowohl turbulente als auch molekulare Transportprozesse von gleichrangiger Größenordnung auftreten. Diese Schicht kann eine Dicke bis zu einigen Dezimetern erreichen. Beide bisher genannten Schichten sind nicht in jedem Falle (z.B. nicht bei hohen Windgeschwindigkeiten über den Ozeanen) ausgebildet [38]. Die untersten 10 \% der Grenzschicht bis zu einer Höhe von 100 m werden als Bodenschicht oder Prandtl-Schicht bezeichnet. Innerhalb dieser Schicht lassen sich die Profile der horizontalen Windgeschwindigkeit, der potentiellen Temperatur, der spezifischen Feuchte und anderer passiver, das Stromfeld nicht beeinflussender Größen mittels universeller Funktionen beschreiben (Monin-Obuchovsche Ähnlichkeitstheorie). Die wichtigsten Eigenschaften dieser Schicht sind der vernachlässigbare Einfluß der großräumigen Druckverteilung und der Erdrotation, was die Beschleunigungen senkrecht zur Windrichtung betrifft, und die Höhenkonstanz der vertikalen Eigenschaftsflüsse. Der erste Aspekt hat eine gegen Null gehende Windrichtungsänderung mit der Höhe in der Prandtl-Schicht zur Folge, da aufgrund der fehlenden Wirkung 


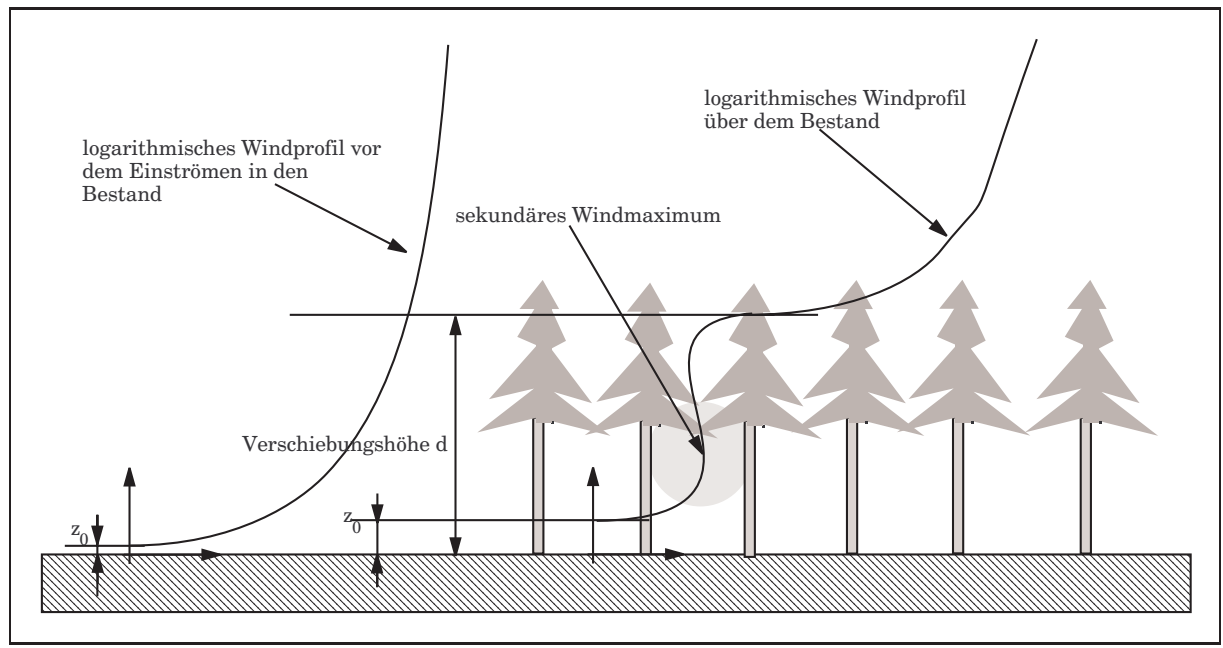

Abbildung 1.4: Schematische Veränderung der Eigenschaftsprofile beim Durch- und Überströmen eines Waldbestandes bei neutraler thermischer Schichtung am Beispiel der horizontalen Windgeschwindigkeit

der Corioliskraft, die Reibungskraft nur eine negative Beschleunigung tangential zur Windrichtung zur Folge hat. Für eine neutrale thermische Schichtung lassen sich die Profile atmosphärischer Eigenschaften entsprechend der Ähnlichkeitstheorie anhand von einfachen logarithmischen Beziehungen beschreiben. So ergibt sich für die horizontale Windgeschwindigkeit $v$ das bekannte logarithmische Windprofil:

$$
v(z)=\frac{v_{*}}{\kappa} \log \left(z / z_{0}\right) .
$$

Dabei ist $z$ die Höhe über der Erdoberfläche, $v_{*}$ die Schubspannungsgeschwindigkeit, $\kappa$ die von Karmán - Konstante $(\kappa=0.4)$ [27]. Die Größe $z_{0}$ beschreibt die Höhe, in der die horizontale Windgeschwindigkeit nach dem logarithmischen Gesetz verschwinden würde. Sie hängt entscheidend von den Oberflächenstrukturen der Unterlage ab und wird deshalb auch als Rauhigkeitslänge bezeichnet. Bei nichtneutraler Schichtung müssen an diese Formel Korrekturterme angebracht werden.

An die Prandtl-Schicht bis zur Obergrenze der atmosphärischen Grenzschicht schließt sich die sogenannte Ekman-Schicht an, die durch eine charakteristische Drehung und Betragsänderung des Windvektors mit der Höhe im Zusammenspiel von Druckgradientkraft, Corioliskraft und Reibungskraft gekennzeichnet ist (Ekman - Spirale) [38].

Die Entwicklung der atmosphärischen Grenzschicht ist einem deutlichen Tagesgang unterworfen. Dieser Tagesgang wird vor allem gesteuert durch die Erwärmung der Erdoberfläche aufgrund der Absorption des nicht reflektierten Anteils der einfallenden Sonnenstrahlung am Erdboden und dem daraus folgenden turbulenten Transport der Wärmeenergie in die darüberliegenden Luftschichten [34].

Der bisher beschriebene mittlere Aufbau der atmosphärischen Grenzschicht 
gilt nicht mehr, wenn zum Beispiel ein Waldbestand über- und durchströmt wird (siehe Abb. 3.2). Hier kommt es zu einer Verschiebung aller Schichten nach oben, die sich mit der Verschiebungshöhe (auch Verdrängungsschichtdicke) beschreiben läßt. Dies ist ein Effekt der durch den Bestand erhöhten Rauhigkeit der Erdoberfläche [38]. Während sich die zähe Unterschicht und die dynamische Unterschicht noch innerhalb des Bestandes direkt an der Erdoberfläche aufgrund ihrer typischen Eigenschaften definieren lassen, wird die Prandtl-Schicht mit ihren typischen logarithmischen Eigenschaftsprofilen um die Verschiebungshöhe nach oben verschoben [38]. Die Profilgesetze in der Prandtl - Schicht ändern sich demnach für neutrale thermische Schichtung, wieder dargestellt am Beispiel der horizontalen Windgeschwindigkeit, zu:

$$
v(z)=\frac{v_{*}}{\kappa} \log \left((z-d) / z_{0}\right),
$$

mit $d$ als Verschiebungshöhe. Für die Größe der Verschiebungshöhe im Verhältnis zur Bestandeshöhe gibt es keine allgemeingültige Gesetzmäßigkeit. Als Orientierung könnte der von Panofsky\&Dutton (1984) angegebene Wert von 70 - $80 \%$ der Bestandeshöhe gelten [1].

Die Profile passiver Eigenschaften innerhalb des Bestandes lassen sich nicht mehr mittels der Ähnlichkeitstheorie beschreiben. Auch für diesen Bereich hat man keine einfache und allgemeingültige Möglichkeit zur analytischen Darstellung der Eigenschaftsprofile gefunden, so daß es sich anbietet, mit numerischen Verfahren zu arbeiten, wie es auch im Rahmen dieser Arbeit geschehen soll.

Eine große Zahl der zum Zwecke der Modellierung der Eigenschaftsflüsse und -profile in der Luft in und über ausgedehnten Waldbeständen benutzten Modelle greifen zur Turbulenzmodellierung auf lokale Schließungsansätze (K-Theorie) zurück ([21],[46],[35], [26]). Für den Transport zwischen der Atmosphäre über dem Bestand und innerhalb des Bestandes erstellte Messungen der auftretenden Fluktuationen der Vertikalbewegung zeigen jedoch ein ganzen Spektrum von Wirbelgrößen, die alle gleichzeitig zum Transport beitragen. Insbesondere große Wirbel mit Abmessungen in der Größenordnung des Bestandes können atmosphärische Eigenschaften aus Höhen über dem Bestand bis in den Stammraum hinein transportieren. So haben Eddy-Kovarianzmessungen an dem $50 \mathrm{~m}$ Meßturm des Institutes für Bioklimatologie in einem Fichtenbestand im Solling im Mai und Juni 1990 ergeben, daß zwischen 9 und $21 \mathrm{~m}$ Höhe innerhalb des $30 \mathrm{~m}$ hohen Bestandes immer noch Wirbel mit Durchmessern größer als $44 \mathrm{~m}$ registriert werden können [5]. Die durchschnittliche Wirbelgröße in diesem Höhenbereich lag zwischen etwa 3.5 und $6.5 \mathrm{~m}$.

Bei der Beschreibung der atmosphärischen Transporte in und oberhalb eines Waldbestandes sollte deshalb der Umstand des Bestehens eines ganzen Spektrums von transportierenden Wirbeln beachtet werden. Da dies mit den verbreiteten lokalen Turbulenzschließungsansätzen nicht möglich ist, soll im Rahmen dieser Arbeit ein nichtlokaler Turbulenzschließungsansatz angewendet werden. 


\section{Kapitel 2}

\section{Lokale Turbulenzschließung: K-Theorie}

Im Rahmen dieser Arbeit wurden parallel zu den Rechnungen mit einem Modell mit transilienter Turbulenzschließung Vergleichsrechnungen mit einem auf lokaler Turbulenzschließung basierenden Modell durchgeführt, welches von der Arbeitsgruppe Mix et al. (siehe auch [26]) zur Verfügung gestellt wurde. Deshalb soll in diesem Kapitel zunächst kurz auf das Turbulenzschließungsproblem im allgemeinen und die lokale Turbulenzschließung im besonderen eingegangen werden.

\subsection{Das Schließungsproblem}

Bei der mathematisch-physikalischen Beschreibung der turbulenten Strömung eines Mediums treten Terme auf, für die sich keine prognostischen oder diagnostischen Gleichungen angeben lassen. Prognostische Gleichungen geben an, wie stark die zeitliche Änderung des Wertes einer physikalischen Größe ist, wobei der aktuelle Wert dieser Größe nicht in jedem Falle vorliegt. Mittels diagnostischer Gleichungen kann der aktuelle Wert einer physikalischen Größe aus den vorliegenden Werten anderer physikalischer Größen berechnet werden. Diese Tatsache wird als Schließungsproblem bezeichnet und soll im weiteren anhand der prognostischen Gleichung für den Windvektor beschrieben werden.

Die lokale zeitliche Änderung der Komponente $u_{i}$ des Windvektors in einer turbulenten Atmosphäre unter Beachtung der Einsteinschen Summenkonvention ${ }^{1}$ und Vernachlässigung von Coriolis- sowie anderen Effekten (z.B. Bodenreibung, konvektiver Transport) läßt sich mittels der folgenden Gleichung beschreiben:

$$
\frac{\partial \overline{u_{i}}}{\partial t}=-\overline{u_{j}} \frac{\partial \overline{u_{i}}}{\partial x_{j}}-\frac{\overline{u_{j}^{\prime} u_{i}^{\prime}}}{\partial x_{j}}
$$

Dabei bezeichnet $u_{i / j}^{\prime}$ die turbulenten Schwankungen der Komponenten, der

\footnotetext{
${ }^{1}$ Über jeden Index der in einem Term zweimal an verschiedenen Stellen auftritt, wird summiert.
} 
obere Querstrich eine zeitliche Mittelung und $\overline{u_{j}^{\prime} u_{i}^{\prime}}$ die Korrelation zwischen $u_{i}^{\prime}$ und $u_{j}^{\prime}$ (statistisches Moment zweiter Ordnung).

Dabei wird vom Taylorschen Ansatz zur Beschreibung der Turbulenz Gebrauch gemacht. Danach kann jede physikalische Größe $S$ in einer turbulenten Strömung dargestellt werden als Summe eines zeitlichen Mittelwertes $\bar{S}$ und der turbulenten Abweichung von diesem Mittelwert $S^{\prime}$, also:

$$
S=\bar{S}+S^{\prime}
$$

Der Mittelungszeitraum wird so gewählt, daß das Mittel über $S^{\prime}$ verschwindet.

Der Term $\overline{u_{j}^{\prime} u_{i}^{\prime}}$ verschwindet für eine laminare Strömung, da hier keine turbulenten Schwankungen existieren, und die Gleichung wird so numerisch lösbar, da nur noch mittlere Größen auftauchen (die Gleichung ist geschlossen). Für die Simulation der mittleren Windgeschwindigkeit in einer turbulenten Strömung muß eine zusätzliche Gleichung für diesen Term gefunden werden. BeRückSichTigt man wiederum nur den advektiven und turbulenten Impulstransport, so erhält man folgende Gleichung [38] S.136 f.:

$$
\frac{\partial \overline{u_{i}^{\prime} u_{j}^{\prime}}}{\partial t}=-\overline{u_{k}} \frac{\partial \overline{u_{i}^{\prime} u_{j}^{\prime}}}{\partial x_{k}}-\overline{u_{i}^{\prime} u_{k}^{\prime}} \frac{\partial u_{j}}{\partial x_{k}}-\overline{u_{j}^{\prime} u_{k}^{\prime}} \frac{\partial u_{i}}{\partial x_{k}}-\frac{\partial \overline{u_{i}^{\prime} u_{j}^{\prime} u_{k}^{\prime}}}{\partial x_{k}} .
$$

Durch Einführung dieser zusätzlichen Gleichung ist das Gleichungssystem aber immer noch nicht geschlossen, da jetzt ein statistisches Moment dritter Ordnung auftritt, für das ebenfalls eine weitere Gleichung eingeführt werden muß. Diese weitere Gleichung würde aber wiederum ein statistisches Moment vierter Ordnung enthalten usw. .

Auf diese „,konventionelle“ Art und Weise läßt sich das Gleichungssystem also nicht lösen (Schließungsproblem). Eine Lösung dieses Problems läßt sich nur finden, wenn man die statistischen Momente unter Verwendung der mittleren Größen parametrisiert (Turbulenzschließung). Die Ordnung der Turbulenzschließung wird durch den Grad der statistischen Momente bestimmt, für die eine Parametrisierung als Näherung eingesetzt wird. Werden die statistischen Momente zweiter Ordnung parametrisiert, so spricht man von einer Schließung erster Ordnung, da in den Gleichungen nur noch mittlere Größen, also statistische Momente erster Ordnung, auftreten. Bei einer Parametrisierung der statistischen Momente dritter Ordnung spricht man von einer Schließung zweiter Ordnung. Da hier jedoch die Momente zweiter Ordnung wiederum parametrisiert werden müssen, steigt die Anzahl der benötigten Gleichungen an. Dies ist der Grund dafür, daß Schließungen erster Ordnung die weiteste Verbreitung in Modellen der atmosphärischen Grenzschicht erreicht haben.

Die Turbulenzschließungsverfahren werden auch nach der Art der Parametrisierung der statistischen Momente klassifiziert. Werden für die Parametrisierung nur lokale Größen (z.B. vertikale Gradienten an einem Ort) verwendet, so spricht man von einer lokalen Schließung, werden jedoch Größen verwendet, die sich aus Werten von Variablen an unterschiedlichen Orten zusammensetzen, so spricht man von einer nichtlokalen Schließung. Zu den lokalen Schließungsansätzen zählt die klassische Form der K-Theorie, die im folgenden beschrieben wird, während die Transiliente Turbulenztheorie zu den nichtlokalen Schließungen gehört. 


\subsection{K-Theorie als lokales Schließungsverfahren}

Bei der Ausbreitung eines Stoffes in einem anderen durch Diffusion zeigt es sich, daß die Ausbreitung und damit der Diffusionsfluß des Stoffes parallel zum Konzentrationsgradienten erfolgt und die Größe des Flusses der Stärke des Gradienten proportional ist. Der Proportionalitätsfaktor wird als Diffusionskoeffizient bezeichnet. Die Diffusion erfolgt immer in Richtung der geringeren Stoffkonzentration, also entgegen dem Konzentrationsgradienten. Sie ist damit immer so gerichtet, daß sich Konzentrationsgegensätze ausgleichen. Dieser Grundsatz wird in Anlehnung an die Theorie des chemischen Gleichgewichts auch als Le Chatelier Prinzip bezeichnet. Nimmt man nun an, eine ähnliche Beziehung ließe sich auf turbulente Flüsse übertragen, so erhält man für den turbulenten Fluß in vertikaler Richtung ( die Komponente der horizontalen Geschwindigkeitsfluktuation wird jetzt mit $u^{\prime}$ und die der vertikalen Geschwindigkeitsfluktuation mit $w^{\prime}$ bezeichnet, $\mathrm{z}$ bezeichnet die Vertikalkoordinate):

$$
\overline{u^{\prime} w^{\prime}}=-K \frac{\partial u}{\partial z} .
$$

Der Proportionalitätsfaktor K (Einheit: $\mathrm{m}^{2} / \mathrm{s}$ ) wird als turbulenter Diffusionskoeffizient bezeichnet. $\mathrm{K}$ kann aus Messungen bestimmt werden oder es wird eine Parametrisierung verwendet.

Für die Parametrisierung von K gibt es verschiedene Ansätze. Der wohl bekannteste ist der Prandtl'sche Mischungswegansatz. Prandtl (1925) führte den Mischungsweg $l$ als Distanz ein, die ein Luftpaket in der Vertikalen zurücklegen muß, um sich an seine Umgebung anzupassen. Mit Hilfe dieses Mischungsweges läßt sich der turbulente Diffusionskoeffizient auf den vertikalen Gradienten der mittleren horizontalen Windgeschwindigkeit zurückführen:

$$
K=l^{2}\left|\frac{\partial \bar{u}}{\partial z}\right| .
$$

Das hat den Vorteil, daß K um so größer wird, je größer der vertikale Gradient der Geschwindigkeit wird, was den Erwartungen entspricht. Für die Mischungsweglänge gibt es ebenfalls verschiedene Ansätze. In Bodennähe wird die mittlere Größe der Wirbel durch das Vorhandensein des Erdbodens beschränkt. Für diesen Fall hat sich eine Parametrisierung aus der technischen Strömungslehre auch in der Meteorologie durchgesetzt:

$$
l=\kappa z .
$$

Dabei ist $\kappa$ die van-Karman-Konstante. Für sie werden Werte von 0.40 bis 0.43 angegeben, in den meisten Fällen wird ein Wert von 0.41 verwendet. Diese Beziehung gilt nur für eine neutrale thermische Schichtung, anderenfalls müssen modifizierte Formeln verwendet werden. Für die freie Atmosphäre konnte sich keine einheitliche Parametrisierung durchsetzen. In diesem Falle müssen sehr viele Effekte Berücksichtigung finden (thermische Schichtung, Corioliseffekte usw.) .

Diese Parametrisierung hat jedoch den Nachteil, daß $K$ nur vom vertikalen Gradienten der horizontalen Windgeschwindigkeit abhängt und nicht von vertikalen Gradienten anderer Größen, insbesondere der Temperatur, die auch 
die Stabilität der Atmosphäre bestimmen. Deshalb werden in atmosphärischen Grenzschichtmodellen oft andere Parametrisierungen für $K$ angewendet.

Eine Möglichkeit ist die sogenannte K-l-b - Schließung, bei der $K$ unter Verwendung der turbulenten kinetischen Energie $b$ und des Mischungsweges $l$ berechnet wird:

$$
K=l \sqrt{b} .
$$

Dabei läßt sich $b$ nach der Vorgabe eines Profiles zu Beginn der Simulation nach der folgenden prognostischen Beziehung berechnen (sie leitet sich aus der Bilanz der kinetischen Energie für jede Modellschicht ab) :

$$
\begin{array}{r}
\frac{\partial b}{\partial t}=-s w \frac{\partial b}{\partial z}+K\left[\left(\frac{\partial u}{\partial z}\right)^{2}+\left(\frac{\partial v}{\partial z}\right)^{2}\right] \\
+\frac{g}{\bar{T}}\left[\frac{S_{H}}{\rho c_{p}}+0.608 T \frac{L_{H}}{\rho}\right]+\alpha_{b} \frac{\partial}{\partial z}\left(K \frac{\partial b}{\partial z}\right)-\alpha_{\epsilon} \frac{b^{2}}{K} .
\end{array}
$$

Dabei ist $T$ die thermodynamische Temperatur der Luft, $c_{p}$ die spezifische Enthalpie der Luft, $\rho$ die Luftdichte sowie $S_{H}$ der sensible und $L_{H}$ der latente Wärmestrom. Die Mischungsweglänge wird dann nach:

$$
l=\frac{\sqrt{b}}{(1+a z)}\left[\frac{l\left(z_{0}\right)}{\sqrt{b_{0}}}+c \frac{\kappa}{2} \int_{z_{0}}^{z} \frac{d z^{\prime}}{\sqrt{b}}\right] \quad \text { mit a }=0 \text { oder } \mathrm{a}=\frac{\kappa|\mathrm{f}|}{0.00027 \mathrm{v}_{\mathrm{g} 0}}
$$

berechnet, wobei $z_{0}$ die Rauhigkeitslänge und $b_{0}$ die turbulente kinetische Energie in der Höhe $z_{0}$. Dieser Ansatz geht zurück auf Blackadar (1962), genannt in [9]. Diese Beziehung kann im Gegensatz zum einfachen Prandtl'schen Mischungswegansatz für die gesamte atmosphärische Grenzschicht angewendet werden. Während in Bodennähe $a=0$ verwendet wird, liefert der zweite Ansatz für $a$ in größeren Höhen bessere Ergebnisse.

Die Wirkung von Waldbeständen kann im Rahmen der K-Schließung durch Anbringen von Zusatztermen in den Gleichungen zur Berechnung von K berücksichtigt werden. Diese Zusatzterme wurden oft empirisch gefunden und sind somit unter physikalischen Gesichtspunkten häufig schwierig nachvollziehbar (siehe z.B. Faktor $a$ in Gl. 2.9). Bezogen auf die K-L-b - Schließung, muß dabei nur die Beziehung zur Berechnung der Turbulenzenergie $b$ innerhalb eines Bestandes modifiziert werden. Die prognostische Gleichung für der turbulente kinetische Energie $b$ erhält im Bestand einen Zusatzterm der Form:

$$
+c_{d} s\left(v_{x}^{2}+v_{y}^{2}\right)^{3 / 2} .
$$

Die Mischungsweglänge ändert sich nach Gleichung 2.9 ebenfalls innerhalb des Bestandes. Alle anderen Modifikationen, die durch den Wärme- und Feuchteaustausch zwischen der Vegetation und der Bestandsluft notwendig werden, sind nicht vom Schließungsansatz abhängig und werden deshalb gesondert im Kapitel 5 behandelt.

Für viele Anwendungen hat sich der K-Schließungsansatz bewährt. Unter bestimmten Bedingungen treten jedoch vertikale turbulente Flüsse auf, ohne daß ein lokaler vertikaler Eigenschaftsgradient vorhanden ist (z.B. freie Konvektion), in diesen Fällen müssen kompliziertere Schließungsansätze verwendet 
werden. Auch bei der Modellierung des turbulenten Austausches in Waldbeständen weichen die Modellergebnisse bei der Verwendung dieses einfachen lokalen Schließungsansatzes mitunter stark von den Meßwerten ab. Der Grund hierfür liegt darin, daß in der turbulenten Atmosphäre verschiedene, unterschiedlich große Wirbel mit unterschiedlich starker Intensität gleichzeitig auftreten, der Prozeß der Turbulenz sich also eindeutig vom Prozeß der Diffusion unterscheidet.

So werden in Waldbeständen oft Eigenschaftsflüsse gemessen, die nach der mathematischen Definition des Eigenschaftsgradienten diesem gleichgerichtet sind und somit dem allgemeinen Verständnis zu widersprechen scheinen. Solche Flüsse werden als ",counter-gradient" - Flüsse bezeichnet, da sie entgegen dem meteorologischen Gradienten gerichtet sind. Eine mögliche Erklärung liefern empirische Spektren der Wirbelgrößenverteilung im Waldbestand. Diese Spektren zeigen, daß auch im Kronen- und Stammraum eines Waldbestandes relativ große Wirbel (> $10 \mathrm{~m}$ Durchmesser) vorkommen. Man nimmt an, daß die mit diesen Wirbeln verbundenen turbulenten Flüsse, die Flüsse aufgrund der kleineren Wirbel überlagern und es so zu diesen scheinbar widersprüchlichen Effekten kommt. 


\section{Kapitel 3}

\section{Transiliente Turbulenztheorie}

\subsection{Einführung}

In diesem Kapitel soll die Transiliente Turbulenz Theorie (TTT) als nichtlokales Turbulenzschließungsverfahren erster Ordnung für den allgemeinen Fall der Modellierung einer turbulenten atmosphärischen Grenzschicht ohne Waldbestand näher erläutert werden. Auf die Modifizierungen dieses Schließungsansatzes, die bei der Grenzschichtmodellierung mit Waldbestand beachtet werden müssen, wird in Kapitel 4 eingegangen.

Das Wort "transilient" bedeutet überspringen und erinnert damit an die nichtlokale Form dieses Schließungsverfahrens. Diese Theorie beruht auf der nichtlokalen Interpretation der prognostischen Gleichung für die Bilanz der turbulenten kinetischen Energie. Die hier beschriebene diskrete Form der TTT ist an ein raum-zeitliches Modellgitter gebunden. Deshalb kann mittels der TTT der turbulente Austausch nur über einen gewissen Zeitraum (Modellzeitschritt) beschrieben werden, der angegeben werden muß. Veröffentlichungen zum Thema Transiliente Turbulenztheorie gibt es ab 1984 ([Stull84,88,90,91,93], [36], [42],[11]).

R. B. Stull [37] bietet eine theoretisch sehr eingehende Einführung in die Grundlagen der TTT. Auch davor gab es Ansätze in Richtung von nichtlokalen Turbulenzschließungen, z. B. die spektrale Diffusionstheorie.

Bei der Beschreibung des turbulenten Transportes in einer Dimension (Vertikalkoordinate) mittels TTT wird der Zustand einer konservativen Variable S (z. B. potentielle Temperatur, spezifische Feuchte oder Windgeschwindigkeit) bestimmt durch den "Grund" -Zustand der Variable vor einem bestimmten Zeitraum und durch die Summe über alle mechanisch und thermisch getriebenen vertikalen turbulenten Flüsse innerhalb des turbulenten Gebietes während dieses Zeitraumes, welche den Grundzustand verändern. Betrachtet man den Vorgang der turbulenten Durchmischung in einem vertikal aufgelösten „raum“ -zeitlich diskretisierten Modell während des Zeitschrittes $\Delta t$, dann ergibt sich der Zustand der Variable in der Gitterzelle i (im folgenden Zielschicht genannt) 
zum Zeitpunkt $t+\Delta t$ als:

$$
S_{i}(t+\Delta t)=\sum_{j=1}^{N} c_{i j}(t, \Delta t) S_{j}(t) .
$$

Dabei ist $\mathrm{N}$ die Gesamtanzahl der Modellschichten und $c_{i j}{ }^{1}$ sind die Koeffizienten einer quadratischen Matrix (der Transilienzmatrix) mit der Dimension $\mathrm{N} \times \mathrm{N}$. Der erste untere Index bezeichnet immer die Nummer der Zielschicht, der zweite immer die der Quellschicht. Die Zielschicht i enthält also eine Mischung von Luftpaketen, die aus unterschiedlichen Quellschichten $\mathrm{j}$ stammen. Welche Schichten das sind, und wie stark die Durchmischung ist, das wird durch die Koeffizienten der Transilienzmatrix $\left(c_{i j}\right)$ bestimmt.

Da die turbulenten Transporte zwischen den Schichten nur mit endlicher Geschwindigkeit erfolgen, ist klar, daß die Koeffizienten $c_{i j}$ vom Zeitschritt abhängen müssen, da in einer kürzeren Zeit auch weniger Luft von der Schicht j in die Schicht i gelangen kann; es ergibt sich dadurch aber auch eine Abhängigkeit vom Aufbau des Modellgitters (Abstände zwischen den Modellschichten) [37]. Bei $\Delta t=0$ ergibt sich zudem eine Matrix mit den Koeffizienten $c_{i j}=1$ für $\mathrm{i}=\mathrm{j}$ und $c_{i j}=0$ sonst (die Einheitsmatrix), da dann keine Luftpakete mehr ausgetauscht werden können. Eine solche Matrix würde somit keine Information über den Prozeß der Turbulenz enthalten, so daß eine Zeitschritt von $\Delta t=0$ z.B. für die Beschreibung des augenblicklichen Zustands der Atmosphäre nicht herangezogen werden kann. Wie bei einem Markov-Prozeß ${ }^{2}$ nimmt man bei der TTT an, daß der augenblickliche Zustand einer Eigenschaft nur von dem Zustand vor Verstreichen einer Zeitspanne $\Delta t$, nicht aber von länger zurückliegenden Zuständen abhängt, die Turbulenz hätte demnach also keine „Erinnerung" . Die Transilienzmatrix beschreibt den turbulenten Austausch von Luftpaketen zwischen den Modellschichten, also die Turbulenz als Prozeß. Da die Luftpakete charakteristische Eigenschaften mit sich führen (z.B. Impuls der Luftbewegung), kann der Austausch von Luftpaketen zur Modellierung der vertikalen Profile von luftgetragenen Eigenschaftsgrößen herangezogen werden. Dabei ist die Struktur der Transilienzmatrix für unterschiedliche Turbulenzbedingungen verschieden, kann also bei instationärer Turbulenz zeitabhängig sein. In einem raum-zeitlich diskretisierten Modell ergibt sich so für jeden Zeitschritt jeweils eine Transilienzmatrix zur Beschreibung des Austauschprozesses, aber verschiedene Profile $\vec{S}$ für die luftgetragenen Eigenschaften.

Die Definition der Koeffizienten der Transilienzmatrix orientiert sich streng an den Zielschichten, d.h. $c_{i j}$ ist der Anteil an der Luftmasse der Schicht i, welcher aus der Schicht $\mathrm{j}$ stammt. Wegen der Zuordnung der ausgetauschten Massen an Luft zu den Koeffizienten $c_{i j}$, müssen sich auch die in Gleichung 3.1 eingesetzten Größen immer auf die Luftmasse beziehen. Diese Bedingung ist bezüglich der potentiellen Temperatur (charakterisiert den Wärmeinhalt pro Masseneinheit), der spezifischen Feuchte (Masse an Wasserdampf pro Masseneinheit) und bei der Windgeschwindigkeit (Impuls pro Masseneinheit) automatisch erfüllt, andere Größen müssen evtl. erst umgerechnet werden. Der

\footnotetext{
${ }^{1}$ Tiefgestellte Indizes bezeichnen die Nummern der Modellschichten

${ }^{2}$ Markov-Prozeß: Zufallsprozeß, bei dem die Wahrscheinlichkeit des Übergangs von einem Zustand in einen anderen nur vom augenblicklichen Zustand abhängt.
} 
Wert der Koeffizienten bezieht sich immer auf die Zielschicht. Insbesondere ist $c_{i j}$ nicht der Anteil der Luft in der Quellschicht j, der in die Zielschicht $\mathrm{i}$ transportiert wird!

An die Koeffizienten der Transilienzmatrix sind einige physikalisch begründete Bedingungen geknüpft, die mathematisch als Randbedingungen der Matrix aufgefaßt werden können. Zunächst muß die Einhaltung der Kontinuitätsgleichung gewährleistet sein. Die Kontinuitätsgleichung kommt in 2 Bedingungen an die Transilienzmatrix zum Ausdruck.

Die erste Bedingung ergibt sich daraus, daß sich die Masse an Luft einer jeden Modellschicht allein durch den turbulenten Austausch mit anderen Schichten nicht verändern darf. Das heißt, die gleiche Masse von Luft, die während eines Zeitschrittes $\Delta t$ aus einer Quellschicht $\mathrm{j}$ in jede Zielschicht $\mathrm{i}$ transportiert wird, muß der Quellschicht wieder zugeführt werden. Setzt man (zunächst für ein äquidistantes Modellgitter) die Luftmasse jeder Schicht auf $1 \hat{=} 100 \%$, so muß die Summe über alle Transporte aus allen Quellschichten $\mathrm{j}$ in die Zielschicht i, ausgedrückt durch die Koeffizienten der Transilienzmatrix $c_{i j}$, für jede Zielschicht genau 1 ergeben. Setzt man diese erste Bedingung in eine mathematische Form um, so erhält man:

- Für jede Zielschicht i (Matrixreihe):

$$
\sum_{j=1}^{N} c_{i j}=1 .
$$

Die zweite Bedingung, die ebenfalls auf die Kontinuitätsgleichung zurückzuführen ist, hängt mit den Werten („Konzentrationen“ = spezifischer Gehalt) der mit der Luft transportierten atmosphärischen Eigenschaften zusammen. Die Werte dieser Konzentrationen können sich im Gegensatz zur Masse an Luft für eine Schicht i infolge der turbulenten Durchmischung sehr wohl ändern, aber aus jeder Quellschicht j kann nicht mehr als 100\% $\hat{=} 1$ einer Eigenschaft heraustransportiert werden. Wieviel einer Eigenschaft aus einer Quellschicht $\mathrm{j}$ in alle Zielschichten i transportiert wird, wird durch die Summe über alle Koeffizienten der Transilienzmatrix $c_{i j}$ über i ausgedrückt. Die mathematische Formulierung dieser zweiten Bedingung lautet somit:

- Für jede Quellschicht j (Matrixspalte):

$$
\sum_{i=1}^{N} c_{i j}=1 .
$$

Da dies für jede Eigenschaft gelten muß, soll diese Bedingung allgemein als Konzentrationserhaltung bezeichnet werden. Eine Matrix, welche den beiden oben genannten Bedingungen genügt, d.h. mathematisch, deren Zeilenelemente bzw. Reihenelemente sich zu 1 summieren. wird in der Mathematik als doppelt stochastisch bezeichnet. Die mathematische Beschreibung der zweiten Bedingung (Konzentrationserhaltung) ist nur richtig, wenn alle Modellschichten die gleiche Höhe besitzen. Da es jedoch vor allem aus Gründen der Recheneffizienz oft erwünscht ist, mit unterschiedlich hohen Modellschichten arbeiten zu können, muß die obige Formel modifiziert werden. 
Die Anpassung der Transilienzmatrix an nichtäquidistante Modellschichten kann durch die Einführung von relativen Luftmassen geschehen [31]. Mit dem Massenverhältnis $\frac{m_{i}}{m_{j}}$ der Schichten i und j ergibt sich als Bedingung:

$$
\sum_{i=1}^{N} \frac{m_{i}}{m_{j}} c_{i j}=1 \quad \text { bzw. } \quad \mathrm{m}_{\mathrm{j}}=\sum_{\mathrm{i}=1}^{\mathrm{N}} \frac{\mathrm{m}_{\mathrm{i}}}{\mathrm{m}_{\mathrm{j}}} c_{\mathrm{ij}} .
$$

Die Massen der Luftschichten können in einem eindimensionalen vertikal aufgelösten Modell in diesem Zusammenhang durch die Schichthöhen ersetzt werden, da die Einflüsse der Luftdichten durch die Verwendung der konservativen Größe potentielle Temperatur Berücksichtigung finden werden (siehe Abschn. 3.2).

Die Gültigkeit dieser Beziehung läßt sich durch eine Beispielbetrachtung erläutern ([31], [41]). In Abb. 3.1 (a) und (b) sind die Schichteinteilung eines äquidistanten Modells, der turbulente Austausch zwischen den Schichten und die dazugehörige Matrix schematisch dargestellt. Indem man nun einige Schichten zusammenfaßt erhält man das Modellgitter für ein nichtäquidistentes Modell (c). Beschreibt dieses Modell nun denselben Austauschprozeß, so ergibt sich eine mit der Matrix in (b) korrespondierende Transilienzmatrix. Es ergeben sich jedoch Unterschiede in der Struktur der Matrix. WährEnd im Falle (a) die Luftmasse der Schicht d die der Schicht a vollständig ersetzt und umgekehrt, wird im Fall (b) die Luftmasse der Schicht 3 nur zu einem Drittel durch die Luftmasse der Schicht 1 ersetzt. Das ergibt einen Wert 0.33 für den Transilienzkoeffizienten $c_{31}$. Umgekehrt wird jedoch wie im Falle (a) die Luftmasse der Schicht 1 vollständig durch Luft der Schicht 3 ersetzt werden $\left(c_{13}=1\right)$. Es ergibt sich also eine gewisse Asymmetrie der Transilienzmatrix (d), obwohl der Prozeß des Austausches durchaus symmetrisch erfolgt (die Transporte von oben nach unten bzw. von unten nach oben halten sich die Waage). Obwohl die Luftmassenund Konzentrationserhaltung erfüllt ist, unterscheiden sich die Summen der Spalten der Matrix (d) von 1. Nur unter Benutzung einer Wichtung der Matrixkoeffizienten jeder Spalte mit dem Verhältnis der Luftmassen bzw. Schichtdicken bei der Summation wie in Gl. 3.4 ergibt sich wieder ein Wert von 1. Für den Fall der 4. Spalte der Matrix (d) ergibt sich für die Summe der Spaltenelemente $\Sigma$ (Masse der untersten Schicht sei gleich einer Masseneinheit):

$$
\begin{gathered}
\Sigma=\frac{m_{1}}{m_{4}} c_{14}+\frac{m_{2}}{m_{4}} c_{24}+\frac{m_{3}}{m_{4}} c_{34}+\frac{m_{4}}{m_{4}} c_{44} \\
=\frac{1}{4} \cdot 0+\frac{2}{4} \cdot 0.50+\frac{3}{4} \cdot 0.6 \overline{6}+\frac{4}{4} \cdot 0.25=1 .
\end{gathered}
$$

Vergleicht man den Turbulenzschließungsansatz der K-Theorie mit der TTT, so kann man die K-Theorie als Spezialfall der TTT auffassen, da sie von der Annahme ausgeht, daß eine turbulente Durchmischung nur zwischen den unmittelbar benachbarten Modellschichten auftritt, die sich durch einen Eigenschaftsgradienten auszeichnen. Das läuft auf eine tridiagonale „Transilienzmatrix" hinaus. 


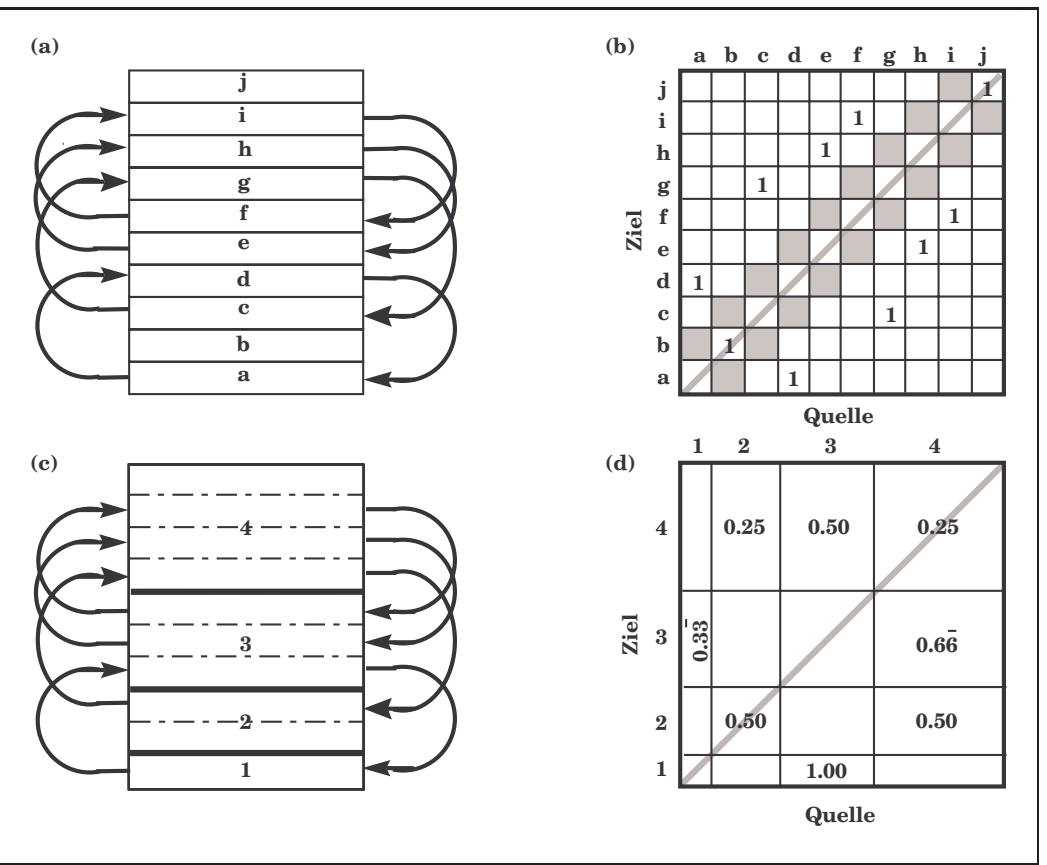

Abbildung 3.1: Schematische Darstellung zweier Transilienzmatrizen für denselben atmosphärischen Prozeß für äquidistante und nichtäquidistante Einteilung der Modellschichten nach [31]. Die linke Seite zeigt, wie der Austausch zwischen den einzelnen Atmosphärenschichten erfolgt. Die rechte Seite zeigt die entsprechenden Transilienzmatrizen. Aus Gründen der besseren Anschaulichkeit wird angenommen, daß die Atmosphärenschichten ihre Massen vollständig austauschen. Zu beachten ist, daß die Matrizen ,auf den Kopf gestellt" wurden, so daß die Zielindizes mit der Höhe der Schichten auf der linken Seite der Abb. korrespondieren. Obwohl die Matrix für die äquidistante Schichteinteilung symmetrisch ist, weist die korrespondierende Matrix für nichtäquidistante Schichteinteilung eine Asymmetrie auf. Die Summe jeder Spalte unterscheidet sich jetzt von 1, obwohl die Bedingungen der Luftmassen- und Konzentrationserhaltung erfüllt sind.

\subsection{Das Mischungspotential}

Die Koeffizienten der Transilienzmatrix hängen vom Potential zur Durchmischung (Mischungspotential) zwischen übereinanderliegenden Atmosphärenschichten ab. Vor der Herleitung des Mischungspotentials sollen zunächst die Struktur und in einer konvektiven Grenzschicht stattfindenden Prozesse näher betrachtet werden, da dies alle Vorgänge einschließt, die bei der atmosphärischen Turbulenz eine Rolle spielen.

\subsubsection{Konvektive Grenzschicht}

Wichtig im Zusammenhang mit der Diskussion unterschiedlicher Schließungsansätze für die Turbulenz in der Atmosphäre ist der Begriff der konvektiven Grenzschicht. Als Konvektion (lat. convehere, convectum = zusammenbringen) wird in der Meteorologie das Auftreten vertikaler Luftbewegungen als 
Folge von starken vertikalen Umlagerungen von Luftpaketen bezeichnet. Erfaßt die Konvektion die gesamte Grenzschicht, so spricht man von einer konvektiven Grenzschicht. Ursachen für die Ausbildung von Konvektion sind örtliche Dichteunterschiede der Luft (z. B. durch das Aufsteigen warmer und gegenüber der Umgebung spezifisch leichterer Luftpakete) aber auch dynamische Instabilität (aufgrund starker vertikaler Scherung des Horizontalwindes kann Turbulenz verursacht werden). Je nach thermischer Schichtung können sich beide Effekte summieren aber auch entgegengesetzt wirken. So kann sich bei thermisch labiler Schichtung in jedem Falle Konvektion ausbilden, wenn dynamische Instabilitäten auftreten, da die durch die dynamische Instabilität hervorgerufenen Vertikalbeschleunigungen von Luftpaketen eine Verstärkung erfahren. Bei thermisch stabiler Schichtung hängt das Auftreten von Konvektion vom Verhältnis der Stärke beider Effekte zueinander ab.

Mathematisch wird dieses Verhältnis durch die Richardson-Zahl ausgedrückt, die im folgenden hergeleitet werden soll.

\subsubsection{Turbulente kinetische Energie}

Entsprechend dem Reynold'schen Ansatz ${ }^{3}$ zur Beschreibung der Turbulenz ergibt sich folgende Beziehung für die turbulente kinetische Energie pro Masseneinheit:

$$
\bar{E}=\frac{1}{2}\left(\overline{u^{\prime 2}}+\overline{v^{\prime 2}}+\overline{w^{\prime 2}}\right) .
$$

Der Querstrich bezeichnet hierbei eine zeitliche Mittelung, während der Apostroph die Abweichungen von den Mittelwerten der jeweiligen Größen kenn-

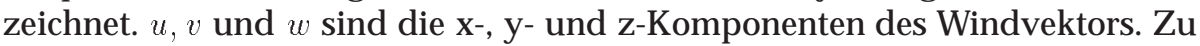
beachten ist hierbei, daß die Mittelung über die Quadrate der zeitlichen Abweichungen nicht zwangsläufig Null ergibt. Die lokale zeitliche Änderung der turbulenten kinetischen Energie in einer Atmosphärenschicht ergibt sich durch lokale Ableitung der obigen Gleichung nach der Zeit und Einsetzen der prognostischen Gleichungen für die Geschwindigkeitsfluktuationen. Nimmt man eine horizontale Strömung (die Vertikalkomponente des mittleren Geschwindigkeitsvektors kann in einem 1-D Modell nicht betrachtet werden) und für die turbulente kinetische Energie horizontale Homogenität an, dann ergibt sich die folgende prognostische Gleichung für die turbulente kinetische Energie [38]:

$$
\begin{gathered}
\frac{\partial E}{\partial t}=\underbrace{-\left(\overline{u^{\prime} w^{\prime}} \frac{\partial \bar{u}}{\partial z}+\overline{v^{\prime} w^{\prime}} \frac{\partial \bar{v}}{\partial z}\right)}_{\mathrm{I}}+\underbrace{\frac{g}{\overline{\Theta_{\vartheta}} \overline{w^{\prime} \Theta_{v}^{\prime}}}-\underbrace{\frac{\partial\left(\overline{w^{\prime} E^{\prime}}\right)}{\partial z}}_{\mathrm{III}}}_{\mathrm{II}} \\
+\underbrace{\frac{1}{\overline{\bar{\rho}} \frac{\partial\left(\overline{w^{\prime} p^{\prime}}\right)}{\partial z}}}_{\mathrm{IV}}-\underbrace{\epsilon}_{\mathrm{V},}
\end{gathered}
$$

\footnotetext{
${ }^{3}$ Jeder Zustand einer Eigenschaft in einer turbulenten Strömung läßt sich beschreiben als Summe eines zeitlichen Mittelwertes (die Mittelungszeit muß entsprechend den Erfordernissen sinnvoll gewählt werden) und der turbulenten Abweichungen von diesem Mittelwert.
} 
mit:

$\begin{array}{lll}u, v & - & \text { horiz. Windkomponenten }\left[\mathrm{ms}^{-1}\right] \\ \Theta_{v} & - & \text { potentielle Temperatur }[\mathrm{K}] \\ \rho & - & \text { Luftdichte }\left[\mathrm{kg} \mathrm{m}^{-3}\right] \\ g & - & \text { Schwerebeschleunigung }\left[\mathrm{ms}^{-2}\right] \\ \frac{\epsilon}{u^{\prime} w^{\prime}}, \overline{v^{\prime}}, w^{\prime} & - & \text { Dissipation pro Masseneinheit }\left[\mathrm{m}^{2} \mathrm{~s}^{-3}\right]=\left[\mathrm{Jkg}^{-1} \mathrm{~s}^{-1}\right] \\ \frac{w^{\prime} \Theta_{v}^{\prime}}{w^{\prime} E^{\prime}} & - & \text { turbulente Impulsflüsse pro Masseneinheit }\left[\mathrm{m}^{2} \mathrm{~s}^{-2}\right] \\ \frac{w^{\prime} p^{\prime}}{} & - & \text { turbulenter Wärmefluß pro Masseneinheit }\left[\mathrm{K} \mathrm{ms}^{-1}\right] \\ & - & \text { Druckkorrelation }\left[\mathrm{hPa} \mathrm{ms}^{-1}\right] .\end{array}$

Die mit I bezeichneten Terme beschreiben die Entstehung von turbulenter kinetischer Energie durch vertikale Scherung der Komponenten der horizontalen Windgeschwindigkeit. Diese beiden Terme führen zu einer Produktion turbulenter kinetischer Energie, da die Terme $\overline{u^{\prime} w^{\prime}}$ und $\overline{v^{\prime} w^{\prime}}$ (die turbulenten Impulsflüsse) ein negatives Vorzeichen führen. Aufgrund der starken Windgeschwindigkeitsscherung in Bodennähe, aber auch häufig an der Obergrenze der Grenzschicht, leisten die Terme I in diesen Bereichen oft den überwiegenden Anteil an der Produktion der turbulenten kinetischen Energie. Der Term II beschreibt die Wirkung des hydrostatischen Auftriebs auf die Turbulenz.

Term III ist der vertikale turbulente Transport von Turbulenzenergie. Dieser Term drückt nur den Transport von turbulenter kinetischer Energie zwischen verschiedenen Schichten innerhalb der Grenzschicht aus und keine Produktion von turbulenter kinetischer Energie. Bei diesem Term handelt es sich um einen Term 3. Ordnung. Das ergibt sich aus der Definition der turbulenten kinetischen Energie. Für eine Turbulenzschließung 1. Ordnung (bei der Terme 2. Ordnung durch Terme 1. Ordnung parametrisiert werden), kann dieser Term nicht berücksichtigt werden, da es keine prognostische Variable (wie z.B. $u$ und $v$ beim Term I) gibt, deren Ableitung nach der Vertikalkoordinate die Menge der turbulent transportierten Turbulenzenergie repräsentiert.

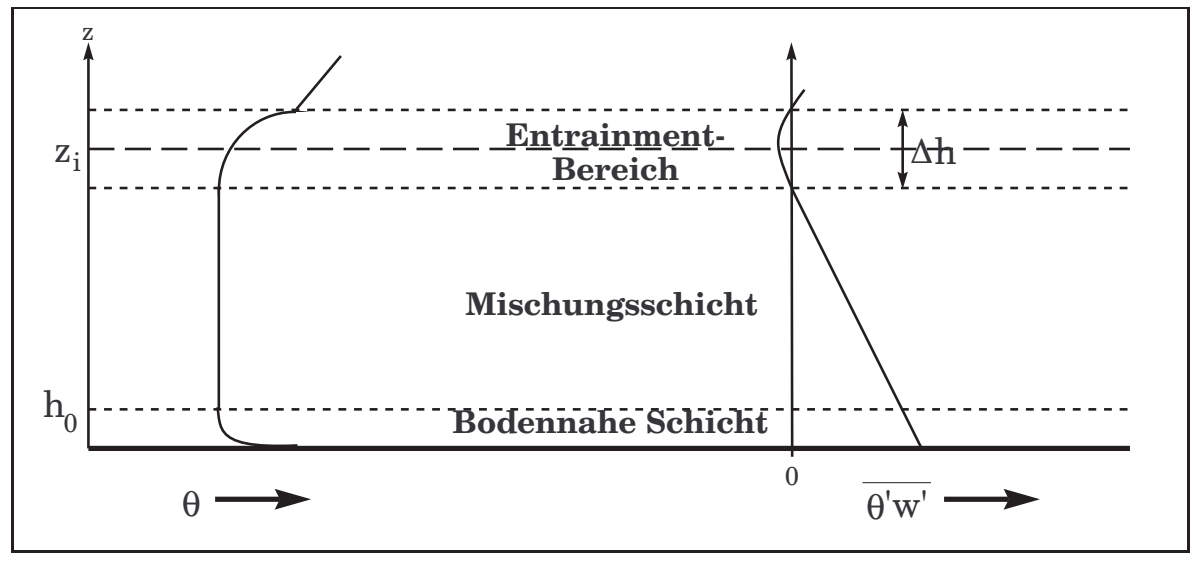

Abbildung 3.2: Schematische Struktur der konvektiven Grenzschicht nach Deardorff, dargestellt anhand der Vertikalprofile der potentiellen Temperatur $\Theta$ und des fühlbaren turbulenten Wärmeflusses $\overline{\Theta^{\prime} w^{\prime}}$. 
Nach Term IV führen auch Abweichungen vom hydrostatischen Gleichgewicht (Fluktuationen des Luftdruckes) zu lokalen Änderungen der Turbulenzenergie. Dieser Term beschreibt die Umverteilung von turbulenter kinetischer Energie zwischen verschiedenen Atmosphärenschichten infolge von Fluktuationen des statischen Druckes (Luftdruck). Das kann z.B. die Folge von Schwerewellen sein. Die dadurch erzeugte turbulente kinetische ist im allgemeinen sehr gering, da die Druckfluktuationen infolge Fluktuationen der Vertikalbewegung sehr gering sind $(0.01 \mathrm{hPa}-0.05 \mathrm{hPa})$ [38]. Über das Verhalten dieses Terms in der Atmosphäre ist nur sehr wenig bekannt, inbesondere kann er nur durch Bilanzierung der turbulenten kinetischen Energie aufgrund von Messungen abgeschätzt werden, indem alle anderen Terme bestimmt werden und diese für eine Strömung mit stationärer Turbulenz zu $\frac{\partial E}{\partial t}=0$ ergänzt werden. Diese Ergänzung ist dann eine Abschätzung für diesen Term. Aufgrund dieser Unsicherheiten, der geringen Bedeutung und eines fehlenden Ansatzes zu seiner Parametrisierung, wird dieser Term im weiteren nicht berücksichtigt.

Term V ist die Dissipation turbulenter kinetischer Energie. Dieser Term weist immer einen von 0 verschiedenen Wert auf, solange die turbulente kinetische Energie ungleich 0 ist.

Die Richardson-Zahl ergibt sich nun als Verhältnis der Änderung der turbulenten kinetischen Energie aufgrund der thermischen Schichtung zur Änderung der turbulenten kinetischen Energie infolge vertikaler Windscherung als Quotient aus den Termen I und II zu [38]:

$$
R_{i}=\frac{g}{\overline{\Theta_{v}}} \overline{w^{\prime} \Theta_{v}^{\prime}}\left[\overline{u^{\prime} w^{\prime}} \frac{\partial \bar{u}}{\partial z}+\overline{v^{\prime} w^{\prime}} \frac{\partial \bar{v}}{\partial z}\right]^{-1} .
$$

Diese Form der Richardson-Zahl hat jedoch den Nachteil, das man ihren Wert nur für turbulente Strömungen bestimmen kann, nicht aber für laminare, da die Terme $\overline{v^{\prime} w^{\prime}}$ und $\overline{u^{\prime} w^{\prime}}$ dann verschwinden. Anhand der Annahmen der lokalen Turbulenzschließung kann man diese turbulenten Flüsse jedoch als proportional zu den Gradienten der jeweiligen Eigenschaft annehmen, was natürlich auch für den Term $\overline{w^{\prime} \Theta_{v}^{\prime}}$ gilt. Setzt man nun diese Gradienten in Gleichung 3.7 ein, so erhält man eine andere Form der Richardson-Zahl, die GradientRichardsonzahl [38]:

$$
R_{i}=\frac{\frac{g}{\Theta_{v}} \frac{\partial \overline{\Theta_{v}}}{\partial z}}{\left(\frac{\partial \bar{u}}{\partial z}\right)^{2}+\left(\frac{\partial \bar{v}}{\partial z}\right)^{2}} .
$$

Das Auftreten von Konvektion läßt sich nun in Abhängigkeit von der Größe von $R_{i}$ beschreiben. Ist der Zähler sehr viel größer als der Nenner, d.h. es herrscht thermisch stabile Schichtung bei geringer vertikaler Windscherung, so liegt eine laminare Strömung vor, da die einzelnen Luftschichten nur horizontal übereinander gleiten können. Der Grenzwert für den Übergang zu einer turbulenten Strömung wird als kritische Richardson-Zahl $R_{c}$ (c - critical) bezeichnet (siehe auch Bulk-Richardsonzahl in Abschnitt 3.2.3). Für die kritische Gradient-Richardson-Zahl wird von vielen Autoren ein Wert von $R_{c}=0.21$ bis $R_{c}=0.25$ angegeben [38]. Die Unterschreitung dieses Wertes reicht allerdings noch nicht aus, um Konvektion auszulösen. Hierzu ist eine noch höhere thermische Instabilität nötig $\left(R_{c}<-1\right.$ [32]). Je nach dem Verhältnis von thermischer Stabilität zu dynamischer Stabilität werden verschiedene Arten 


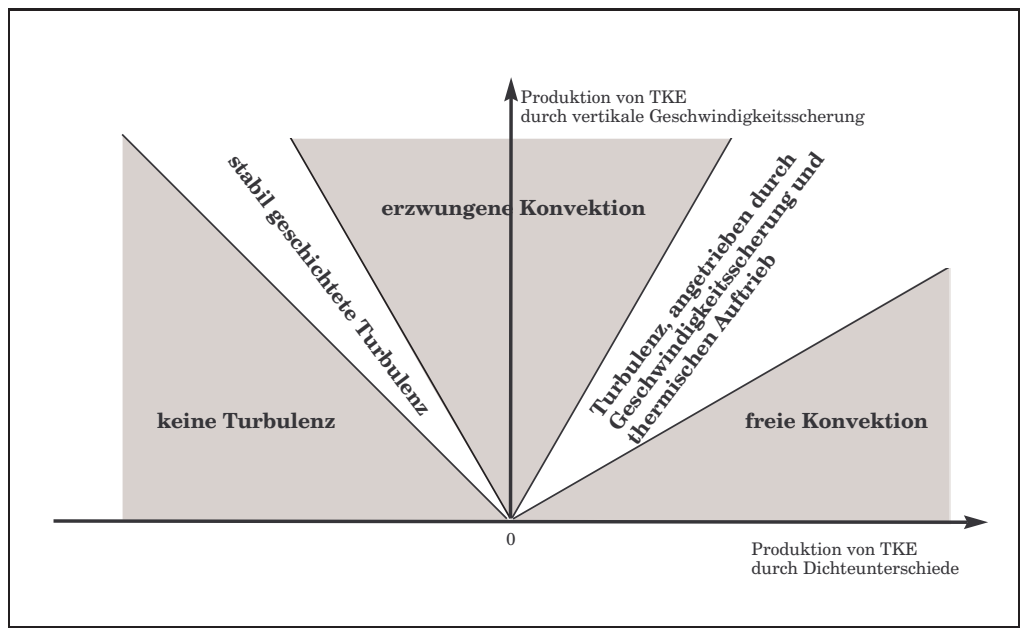

Abbildung 3.3: Auftreten unterschiedlicher Regimes der Turbulenz in der atmosphärischen Grenzschicht in Abhängigkeit von der vorherrschenden Art der Produktion turbulenter kinetischer Energie (TKE) (nach [38]).

der Konvektion unterschieden, wie sie in Abb. 3.3 dargestellt sind. Wenn die thermische Instabilität sehr viel größer ist als die dynamische, so spricht man von freier Konvektion, im umgekehrten Falle von erzwungener Konvektion, womit die 2 Grenzfälle der Konvektion umschrieben sind. Freie Konvektion kann demnach dann auftreten, wenn die Erwärmung der Erdoberfläche sehr stark ist, also vor allem bei sommerlichen Schönwetterperioden. Bei starker Bewölkung und großen Windgeschwindigkeiten wie es z.B. beim Durchzug der Fronten eines Tiefdruckgebietes möglich ist, kann dagegen erzwungene Konvektion auftreten, da die vertikale Windscherung in Bodennähe sehr groß wird. Bei der Gradientrichardsonzahl wird von manchen Autoren ein kritischer Wert von $R_{i}=-10$ angegeben, der unterschritten werden muß, bevor freie Konvektion auftritt [38].

Aufgrund der kinetischen Energie aufsteigender Luftpakete können diese teilweise in den Bereich stabiler oder neutraler Schichtung über der Grenzschichtobergrenze eindringen. Dieser Effekt trägt zum Wachstum der vertikalen Mächtigkeit einer konvektiven Grenzschicht in den Vormittagsstunden bei. Andererseits müssen als Kompensation kältere Luftpakete aus dem Bereich oberhalb der Grenzschicht in den oberen Bereich der Grenzschicht eindringen. Durch diese beiden Prozesse entsteht an der Obergrenze der Grenzschicht ein Bereich, in dem sich Luftpakete aus der Grenzschicht aber auch aus Höhen über der Grenzschichtobergrenze wiederfinden. Dieser Bereich wird deshalb auch als „Entrainment" -Schicht (engl. entrain = einbringen) bezeichnet (Abb. 3.2). 


\subsubsection{Parametrisierung des Mischungspotentials auf der Basis der Richardsonzahl}

Eine Möglichkeit der Parametrisierung des Mischungspotentials und damit auch der Transilienzmatrix beruht auf der Überlegung, daß durch die turbulente Durchmischung Instabilitäten, d.h. in unserem Falle vertikale Eigenschaftsgradienten, soweit ausgeglichen werden könnten, daß die Turbulenz zum Erliegen kommt. Dieser Fall tritt in der Natur kaum auf, da durch vertikal variierende Quellen und Senken atmosphärischer Eigenschaften zeitlich parallel zum Abbau der Instabilitäten immer wieder ein Aufbau dieser Instabilitäten (z.B. vertikale Variation der Reibung als Senke des Impulses und des horizontalen Luftdruckgradienten als Quelle des Impulses) erfolgt. Nähme man jedoch Quellen und Senkenfreiheit an, dann müßte die Turbulenz nach einem gewissen Zeitraum zum Erliegen kommen. Eine gute Möglichkeit zur Beschreibung der zeitlichen Entwicklung des Verhaltens einer turbulenten Atmosphäre bietet die Gradient-Richardsonzahl $R_{i}$ (siehe Gleichung 3.8). Da die lokalen Gradienten der potentiellen Temperatur und der horizontalen Windgeschwindigkeit oft nicht bekannt sind, werden die Gradienten häufig durch Differenzen der entsprechenden Größen über endliche vertikale Distanzen ersetzt (Bulk-Richardsonzahl $R_{b}$ ). Nach Einsetzen der Differenzen und Umstellen erhält man:

$$
R_{b}=\frac{\left(\frac{g}{\Theta}\right) \Delta \Theta \Delta z}{(\Delta u)^{2}+(\Delta v)^{2}} .
$$

Was sagt die Bulk-Richardsonzahl aus? Die Aussagen über den Zusammenhang zwischen Richardsonzahl und Turbulenz beruhen auf empirischen Erfahrungen, denen jedoch physikalische Überlegungen zugrunde liegen.

In einer nichtturbulenten Strömung kommt es zur Ausbildung von Turbulenz, wenn die vertikale Windscherung einen durch die Auftriebskräfte bestimmten Wert überschreitet, d. h. wenn $R_{b}$ einen Wert, den man als kritischen Wert $R_{c}$ (c - critical) der Bulk-Richardsonzahl bezeichnet, unterschreitet. Die entstandene Turbulenz führt zu einer Abschwächung der vertikalen Gradienten. Damit steigt aber die Richardsonzahl wieder an. Dieser Prozeß kann sich ohne Turbulenzquellen so lange erhalten, bis die Turbulenz zum Erliegen kommt. Der Wert der Bulk-Richardsonzahl, bei dem dies geschieht, wird als Abbruchwert $R_{t}$ ( $\mathrm{t}$ - termination) bezeichnet. Es ist zu beachten, daß die Turbulenz nicht erst dann zum Erliegen kommt, wenn die vertikalen Gradienten vollständig verschwunden sind, denn dann wäre $R_{b}$ nicht definiert! Aufgrund des beschriebenen Zusammenhanges zwischen Turbulenz und Richardsonzahl läßt sich diese gut für die Parametrisierung der Turbulenz einsetzen. Dafür müssen jedoch die Werte $R_{c}$ und $R_{t}$ bekannt sein. Für eine vertikale Auflösung von $\Delta \mathrm{z}=50 \mathrm{~m}$ und besser wurden Werte von $R_{c}=0.25$ und $R_{t}=1.0$ angegeben [36]. Für eine Anwendung der Bulk-Richardsonzahl zur Parametrisierung von Transilienzmatrizen muß diese zunächst nichtlokal interpretiert werden [36],[47]:

$$
r_{i j}=\frac{g}{\overline{\Theta_{i j}}} \frac{\left(\Theta_{i}-\Theta_{j}\right)\left(z_{i}-z_{j}\right)}{\left(u_{i}-u_{j}\right)^{2}+\left(v_{i}-v_{j}\right)^{2}} .
$$

$r_{i j}$ ist die nichtlokale Bulk-Richardsonzahl. Die Indizes i bzw. j kennzeichnen die Zugehörigkeit der entsprechenden Größen zu den Schichten i bzw. j. Da- 
bei werden für Windgeschwindigkeit und Temperatur jeweils die Schichtmittelwerte verwendet. $\bar{\Theta}_{i j}$ ist das arithmetische Mittel aus den Mitteltemperaturen der Schichten i und j. Unter der Annahme einer symmetrischen Matrix $\left(r_{i j}=r_{j i}\right.$ wurde folgende Parametrisierung einer "Antriebs" -Matrix gefunden, welche die zeitliche Entwicklung der Turbulenz in unterschiedlichen räumlichen Bereichen beschreibt [47]:

$$
Y_{i j}=w_{i j}\left[1-\frac{r_{i j}}{R_{t}}\right] \text {. }
$$

Dabei ist $R_{t}$ jetzt der Abbruchwert, bei dessen Überschreiten durch die aktuelle Richardsonzahl $r_{i j}$ der turbulente Austausch zwischen den Schichten i und j zum Erliegen kommt. Der Term $w_{i j}$ ist eine Gewichtsfunktion, die die Einflüsse von Gitterweite und Zeitschritt auf die Modellphysik reduzieren soll, mit:

$$
w_{i j}=\frac{U_{0} \Delta t}{z_{j}-z_{i}} \quad \text { für } \mathrm{i} \neq \mathrm{j} .
$$

$U_{0}$ ist eine charakteristische Skalengeschwindigkeit von derselben Größenordnung wie die konvektive Skalengeschwindigkeit $w_{*}$. Die Werte von $w_{i j}$ werden begrenzt („,abgeschnitten“), so daß sie immer zwischen 0 und 1 liegen. Das gleiche gilt für die Werte des Potentials $Y_{i j}$ - dadurch wird Entmischung aus einer Schicht $\left(r_{i j}<R_{i}\right)$ verhindert [47]. Die Transilienzmatrix wird mittels der folgenden Beziehungen definiert:

$$
\begin{gathered}
c_{i j}=\frac{1}{n_{D}} Y_{i j} \quad \text { für } \mathrm{i} \neq \mathrm{j} \\
c_{i i}=1-\sum_{\substack{j=1 \\
j \neq i}}^{N} c_{i j} .
\end{gathered}
$$

Dabei ist $N$ die Gesamtanzahl der Modellschichten und $n_{D}$ ist die Anzahl der Modellschichten innerhalb des turbulenten Bereiches, die zu jedem Zeitschritt neu bestimmt wird. Mit den Werten $U_{0}=0.5 \mathrm{~ms}^{-1}, R_{c}=1.5$ und $R_{t}=2.0$ wurden von Stull [37] für eine Grenzschicht ohne Waldbestand gute Simulationsergebnisse erzielt.

Diese Parametrisierung hat allerdings den Nachteil, daß die erhaltene Transilienzmatrix immer symmetrisch ist. Der in der Natur zu beobachtende turbulente Austausch erfolgt häufig jedoch nicht symmetrisch, inbesondere bei thermischer bedingter freier Konvektion, da dann starke gerichtete Transporte über größere Höhendifferenzen hinweg erfolgen.

\subsubsection{Parametrisierung des Mischungspotentials auf der Basis der Turbulenzenergie}

Die Definition des Mischungspotentials beruht auf einer nichtlokalen Interpretation der kinetischen Energie der turbulenten Zusatzbewegung, wie die im allgemeinen als Turbulenzenergie bezeichnete Größe eigentlich genannt werden müßte. Eine Definition des Mischungspotentials auf der Grundlage Turbulenzenergiegleichung 3.6 wurde von Stull und Driedonks [42] vorgeschlagen. 
Da wir eine nichtlokale Turbulenzschließung erhalten wollen, betrachten wir die Energie $E$ a-priori als Turbulenzenergie $E_{i j}$ des räumlichen Bereiches zwischen $z_{j}$ und $z_{i}$, welche durch Instabilitäten innerhalb dieses Bereiches erzeugt wird. Auch die turbulenten Flüsse werden jetzt nichtlokal interpretiert, d. h. demselben Bereich $z_{j}-z_{i}$ zugeordnet. Nun kann man die partiellen Ableitungen nach der Vertikalkoordinate für die Anwendung in einem numerischen Modellgitter durch die entsprechenden Differenzenquotienten ersetzen und über einen Zeitschritt $\Delta t$ integrieren. Um ein allgemein anwendbares Maß für die zeitliche Änderung der turbulenten Energie zu erhalten, wird noch mit $E_{i j}$ normiert und man erhält so:

$$
\begin{array}{r}
\frac{\Delta_{t} E_{i j}}{E_{i j}}=\left[\frac{-{\overline{u^{\prime} w^{\prime}}}_{i j}}{E_{i j}} \frac{\Delta u_{i j}}{\Delta z_{i j}}+\frac{-{\overline{v^{\prime} w^{\prime}}}_{i j}}{E_{i j}} \frac{\Delta v_{i j}}{\Delta z_{i j}}\right. \\
\left.+\frac{g}{\overline{\Theta_{v, i j}}} \frac{\overline{w^{\prime} \Theta_{v i j}^{\prime}}}{E_{i j}}-\frac{\epsilon_{i j}}{E_{i j}}\right] \Delta_{t} t .
\end{array}
$$

Dabei kennzeichnet $\Delta_{t}$ eine zeitliche Differenz, $\Delta$ eine räumliche Differenz und $\overline{\Theta_{v, i j}}$ das arithmetische Mittel der potentiellen Temperatur und $\bar{\rho}_{i j}$ das arithmetische Mittel der Luftdichte des Bereiches zwischen $z_{j}$ und $z_{i}$. Es wird darauf hingewiesen, daß diese Beziehung unter der a-priori-Annahme gültig ist, daß die Turbulenzenergie entsprechend dem räumlichen Abstand linear in Komponenten zerlegt werden kann [42]. Es müssen nun noch geeignete Parametrisierungen für die Korrelationsterme und den Dissipationsterm gefunden werden. Nach Stull und Driedonks können 3 Skalierungsparameter eingeführt werden: eine Zeitskale der Turbulenz $T_{0}$, ein dimensionsloser Parameter $R_{c}$ (= kritische Richardson-Zahl), der einen Zusammenhang zwischen der thermischen Schichtung und der Windscherung herstellt, und ein dimensionsloser Dissipationsparameter $D$. Diese Parameter werden benötigt, um die Korrelationsterme als Funktionen von Größen 1. Ordnung darzustellen:

$$
\begin{aligned}
\frac{-\overline{u^{\prime} w^{\prime}}}{E_{i j}} & =T_{0} \frac{\Delta u_{i j}}{\Delta z_{i j}} \\
\frac{-\overline{v^{\prime} w^{\prime}}}{E_{i j}} & =T_{0} \frac{\Delta v_{i j}}{\Delta z_{i j}} \\
\frac{-\overline{w^{\prime} \Theta_{v i j}^{\prime}}}{E_{i j}} & =\frac{T_{0}}{R_{c}} \frac{\Delta \Theta_{v, i j}}{\Delta z_{i j}} \\
\frac{\epsilon_{i j}}{E_{i j}} & =\frac{D}{T_{0}} .
\end{aligned}
$$

Die ersten drei Parametrisierungen enthalten Differenzenquotienten, da eine Schließung 1. Ordnung erwünscht ist. Die Vorfaktoren der rechten Seiten ergeben sich aus der Betrachtung der physikalischen Einheiten beider Gleichungsseiten. Auf die Bestimmung der Werte der Vorfaktoren wird später eingegangen. Die vierte Parametrisierung basiert auf der Überlegung, daß die Turbulenz ohne Einwirkung äußerer Kräfte nach einer gewissen Zeit $\left(\sim T_{0}\right)$ durch Dissipation zum Erliegen kommt, und daß die Dissipation umso geringer ist, je 
größer diese Zeit ist. Die linke Seite der Gleichung 3.16 ist ein Maß für die zeitliche Änderung der Turbulenzenergie durch turbulente Durchmischung und kann deshalb als Mischungspotential bezeichnet werden. Damit erhält man die folgende Beziehung für das gesuchte Mischungspotential $Y_{i j}$ :

$$
\begin{array}{r}
\left(Y_{i j}\right)=\frac{T_{0} \Delta_{t} t}{\left(\Delta z_{i j}\right)^{2}}\left[\left(\Delta u_{i j}\right)^{2}+\left(\Delta v_{i j}\right)^{2}\right. \\
\left.-\frac{g}{R_{c} \overline{\Theta_{v, i j}}} \Delta \Theta_{v, i j} \Delta z_{i j}\right]-D \frac{\Delta_{t} t}{T_{0}} .
\end{array}
$$

Für die Diagonalelemente der Matrix des Mischungspotentials wird zunächst ein Wert von $Y_{i i}=0$ angenommen. Das so definierte Potential soll im Gegensatz zu der folgenden Form als dimensionsloses Mischungspotential bezeichnet werden. Diese Parametrisierung hat jedoch den Nachteil, daß sie vom Modellzeitschritt abhängt. Um eine von der Modellcharakteristik unabhängige Parametrisierung des Mischungspotentials zu erhalten, wurde von Stull und Bartnicki [43] eine modifizierte Form der Parametrisierung eingeführt, indem die Gleichung mit $\frac{\left(\Delta z_{i j}\right)^{2}}{T_{0} \Delta_{t} t}$ durchmultipliziert wird und an die Stelle von $Y_{i j} \frac{\left(\Delta z_{i j}\right)^{2}}{T_{0} \Delta_{t} t}$ und $\frac{D}{T_{0}^{2}}$ ein neues $Y_{i j}$ und ein neues $D$ gesetzt wird (die dann auch eine entsprechend andere Einheit erhalten: $\left[Y_{i j}\right]=m^{2} s^{-2} ;[D]=s^{-2}$ ). Damit erhält man für das Mischungspotential die folgende Beziehung:

$$
\left(Y_{i j}\right)=\left(\Delta u_{i j}\right)^{2}+\left(\Delta v_{i j}\right)^{2}-\frac{g}{R_{c} \bar{\Theta}_{v, i j}} \Delta \Theta_{v, i j} \Delta z_{i j}-D\left(\Delta z_{i j}\right)^{2} \quad \text { für } \mathrm{i} \neq \mathrm{j} .
$$

mit:

$$
\begin{array}{lll}
\left(Y_{i j}\right) & - & \text { Mischungspotential } \\
u_{i}, u_{j}, v_{i}, v_{i} & - & \text { Komponenten der horizontalen Windgeschwindigkeit } \\
& & \text { in der Quell- bzw. Zielschicht } \\
\mathrm{g} & - & \text { Schwerebeschleunigung } \\
R_{c} & - & \text { kritische Richardson-Zahl } \\
\Theta_{v, i j} & - & \text { Mittelwert der potentiellen Temperatur } \\
& & \text { zwischen Quell- und Zielschicht. }
\end{array}
$$

WährEnd die ersten zwei Terme der rechten Seite die mechanische Produktion von Turbulenz bzw. Durchmischung beschreiben, steht der dritte Term für die Produktion bzw. Abschwächung der Turbulenz (je nach thermischer Stabilität). Wenn die Summe des dritten und vierten Terms größer ist als die Summe des ersten und zweiten Terms, was bei stabiler Schichtung und geringer vertikaler Windscherung der Fall sein kann, wird dem Mischungspotential der Wert Null zugewiesen. Diese Einschränkung des Wertebereiches ergibt sich aus der Bedingung, daß alle Elemente der späteren Transilienzmatrix $\geq 0$ sein müssen. Transilienzkoeffizienten kleiner Null widersprechen dem Entropiesatz. Die Wirkung negativer Elemente in der Transilienzmatrix kann anhand eines Beipiels demonstriert werden. Die Transilienzmatrix für ein 3-SchichtenModell habe die Form:

$$
\left(\begin{array}{rrr}
1 & 1 & -1 \\
-1 & 1 & 1 \\
1 & -1 & 1
\end{array}\right)
$$


Eine passive skalare Größe habe in der ersten Schicht den Wert 3, in der zweiten Schicht den Wert 2 und in der obersten Schicht den Wert 1. Die Werte dieser Größe nach einem Zeitschritt ergeben sich bei Quellen- und Senkenfreiheit durch Matrixmultiplikation zu:

$$
\left(\begin{array}{rrr}
1 & 1 & -1 \\
-1 & 1 & 1 \\
1 & -1 & 1
\end{array}\right)\left(\begin{array}{l}
1 \\
2 \\
3
\end{array}\right)=\left(\begin{array}{l}
2 \\
0 \\
4
\end{array}\right)
$$

Demnach hätte also eine vollständige Entmischung aus Schicht 2 stattgefunden. Der Entropiesatz besagt jedoch, daß in einem thermodynamischen System bei Ausschluß äußerer Kräfte immer ein Zustand höherer Wahrscheinlichkeit und damit höherer Entropie angestrebt wird. Äußere „Kräfte“ wären in unserem Falle Quellen- und Senken, die jedoch laut Voraussetzung ausgeschlossen wurden. Der nun erreichte Zustand ist aber sehr viel unwahrscheinlicher als der ursprüngliche. Die ermittelte Durchmischung gilt dabei für jede passive skalare Größe, da diese mit den Luftpaketen mittransportiert wird. Das Ergebnis entspricht einer Entropieabnahme im Modellgebiet und widerspricht damit dem Entropiesatz.

Für die neu eingeführten Parameter $R_{c}, T_{0}$ und $D$ für die Parametrisierung des Mischungspotentials nach Gleichung 3.21 wurden von Stull\&Driedonks wurden für eine atmosphärische Grenzschicht ohne Bestand und äquidistantes Modellgitter Testrechnungen mit verschiedenen Werten für diese Parameter durchgeführt [42]. Die folgenden Werte wurden getestet:

$$
\begin{gathered}
-R_{c}=0.21,0.25,1.0 \\
-T_{0}=10 \mathrm{~s}, 100 \mathrm{~s}, 1000 \mathrm{~s} \\
\bullet D=1,10,100 .
\end{gathered}
$$

Dabei wurde eine unterschiedliche Empfindlichkeit der Simulationsergebnisse je nach variiertem Parameter festgestellt. Der Schließungsansatz ist demnach sehr sensitiv in Bezug auf Änderungen des Parameters $R_{c}$ und weniger sensitiv in Bezug auf Änderungen der Parameter $T_{0}$ und $D$. Durch Vergleich der Simulationsergebnisse mit Messungen wurde als beste Kombination der $3 \mathrm{~Pa}-$ rameter gefunden:

$$
R_{c}=0.21, T_{0}=100 \mathrm{~s}, D=1 .
$$

Eine „Erweiterung“ der Parametrisierung des Mischungspotentials haben Stull und Bartnicki 1993 vorgeschlagen [43]. Im dritten Term der Gleichung 3.21 wird dazu für $\frac{\Delta \Theta_{v, i j}}{\Delta z_{i j}}$ ein Term $\frac{\Delta \Theta_{v, i j}}{\Delta z_{i j}}$ eingesetzt, wobei $\Delta z_{i j}^{\prime}$ berechnet wird nach:

$$
\Delta z_{i j}^{\prime}= \begin{cases}z_{i j} & \text { für thermische Stabilität }\left(\frac{\Delta \Theta_{v, i j}}{\Delta z_{i j}} \geq 0\right) \\ h_{1} & \text { für thermische Instabilität }\left(\frac{\Delta \Theta_{v, i j}}{\Delta z_{i j}}<0\right) .\end{cases}
$$

Dabei ist $h_{1}$ die Dicke der untersten Modellschicht. Setzt man diese Beziehung in Gleichung 3.21 ein, so erhält man (für $\mathrm{i} \neq \mathrm{j}$ ):

$$
\left(Y_{i j}\right)=\left(\Delta u_{i j}\right)^{2}+\left(\Delta v_{i j}\right)^{2}-\frac{g}{R_{c} \bar{\Theta}_{v, i j} \Delta z_{i j}^{\prime}} \Delta \Theta_{v, i j}\left(\Delta z_{i j}\right)^{2}-D\left(\Delta z_{i j}\right)^{2} .
$$


Diese abgeänderte Parametrisierung soll das ungehinderte Aufsteigen warmer Luftpakete in der Grenzschicht bei rein thermisch labiler Schichtung (freie Konvektion) simulieren, wie es von Crum\&Stull 1987, gen. in [43], festgestellt wurde. Dabei spielt nur der Unterschied in der potentiellen Temperatur zwischen der Quell- und der Zielschicht eine Rolle, nicht deren Abstand. Das soll durch das Einsetzen von $h_{1}$ anstelle der Höhendifferenz $\Delta z_{i j}$ erreicht werden. Der selbe Effekt betrifft auch die Luftpakete, die in einem Bereich labiler thermischer Schichtung absinken. Auch dieses Absinken erfolgt relativ ungehindert. Für die Rechnungen mit Waldbestand im Rahmen dieser Arbeit wurde diese Form der Parametrisierung nicht angewendet, da die Verwendung von $h_{1}$ eine zusätzliche Abhängigkeit der Parametrisierung von der Einteilung der Modellschichten mit sich bringt. Es hat sich zudem noch gezeigt, daß beim Übergang zur Transilienzmatrix sehr viel mehr Iterationen entsprechend dem Abschnitt 3.4 notwendig waren, wodurch sich die Rechenzeit sehr stark erhöhte.

Das so definierte Mischungspotential ist für äquidistante Modellschichten immer symmetrisch $\left(Y_{i j}=Y_{j i} ; \mathrm{i}, \mathrm{j}=1 \rightarrow \mathrm{N}\right)$, woran auch der die thermische Schichtung ${ }^{4}$ berücksichtigende Term III nichts ändert, da das Vorzeichen und der Betrag dieses Termes für $Y_{i j}$ und $Y_{j i}(\mathrm{i}, \mathrm{j}=1 \rightarrow \mathrm{N})$ jeweils gleich sind. Transilienzmatrizen für eine Grenzschicht ohne Waldbestand, welche aus LargeEddy-Simulationen abgeleitet wurden [11] (siehe auch Abschnitt 3.7), zeigen für äquidistante Modellschichten im Falle freier Konvektion jedoch eine Unsymmetrie. Diese Unsymmetrie ist auf die unterschiedlichen Geschwindigkeiten, mit denen Luftpakete aufwärts bzw. abwärts transportiert werden, zurückzuführen.

Eine Möglichkeit zur Parametrisierung nichtsymmetrischer Transilienzmatrizen für den Fall einer Grenzschicht mit freier Konvektion ist durch die Verwendung der "konvektiv verfügbaren potentiellen Energie“ (engl.: convective available potential energy - CAPE) möglich, wie es Stull 1996 gezeigt hat [44]. Diese verfügbare potentielle Energie läßt sich berechnen nach:

$$
C A P E=\int_{z_{u}}^{z} \frac{g}{\overline{T_{v}}} \Delta \Theta_{v}\left(z^{\prime}\right) d z^{\prime}
$$

Dabei bezeichnet $z_{u}$ die Höhe der unteren Grenze des konvektiven Bereiches der Atmosphäre und $z$ die aktuelle Höhe des Teilchens, $g$ ist die Fallbeschleunigung und $\overline{T_{v}}$ ist die mittlere virtuelle Temperatur der Atmosphäre im Höhenbereich von $z_{u}$ bis $z_{o} . \Delta \Theta_{v}\left(z^{\prime}\right)$ ist die Differenz der potentiellen Temperatur des Luftpaketes zur potentiellen Temperatur der Umgebungsluft. Die Bedeutung von CAPE läßt sich folgendermaßen veranschaulichen:

Die vertikale Beschleunigung $\dot{w}$, die ein Luftteilchen aufgrund des hydrostatischen Auftriebs erfährt, ergibt sich im Gleichgewicht mit der Schwerebeschleunigung $g$ und der vertikalen Komponente des Luftdruckgradienten $\frac{\partial p}{\partial z} \mathrm{zu}$ :

$$
\dot{w}=-g-\frac{1}{\rho} \frac{\partial p}{\partial z} .
$$

\footnotetext{
${ }^{4}$ Auch der Begriff der thermischen Schichtung muß hier nichtlokal interpretiert werden!
} 
Nimmt man für die nächste Umgebung des sich vertikal bewegenden Teilchens hydrostatisches Gleichgewicht an, so kann man, da der Luftdruck des Teilchens $p$ dem Luftdruck der Umgebung $\bar{p}$ entspricht, $\frac{\partial \bar{p}}{\partial z}$ und somit $-g \bar{\rho}$ an Stelle von $\frac{\partial p}{\partial z}$ einsetzen. Durch Umstellen und Anwendung der idealen Gasgleichung $(\rho R T=$ const. für $p=$ const., also $T \sim 1 / \rho)$ erhält man für $\dot{w}$ :

$$
\dot{w}=g \frac{T-\bar{T}}{\bar{T}},
$$

wobei $T$ die Temperatur des Luftteilchens und $\bar{T}$ die Umgebungstemperatur bezeichnet. Da hier die Temperaturdifferenz im selben Niveau gebildet wird, kann dafür die Differenz der potentiellen Temperaturen eingesetzt werden. Die vertikale Beschleunigung entspricht einem Gewinn des Teilchens an spezifischer (auf die Masseneinheit bezogener) potentieller Energie (deshalb verfügbare potentielle Energie). Bewegt sich das Teilchen vertikal, so kann sich die potentielle Temperatur der in gleicher Höhe gelegenen Umgebung ändern. Dieser Effekt wird bei der Vertikalbewegung berücksichtigt, indem die Beziehung für $\dot{w}$ über den zurückgelegten Weg des Teilchens integriert wird. Bei freier Konvektion kann angenommen werden, daß sich das Luftteilchen so schnell vertikal bewegt, daß es einem adiabatischen Prozeß unterworfen wird, so daß seine potentielle Temperatur konstant bleibt, und man erhält die obige Beziehung für CAPE.

Das Mischungspotential ist entsprechend der Gleichung 3.21 für alle Fälle i $\neq \mathrm{j}$ definiert, nicht aber entlang der Diagonalen, d.h. für $\mathrm{i}=\mathrm{j}$. Die Werte $Y_{i i}$ represäntieren die Durchmischung durch Wirbel, deren Durchmesser kleiner ist als die Dicke der entsprechenden Schicht i.

Für die Parametrisierung dieser subskaligen Turbulenz wurden verschiedene Methoden entwickelt. Da sich diese Turbulenz in einem Bereich abspielt, der sich unterhalb der Auflösungsgrenze des numerischen Modells befindet, ist der Weg zur Parametrisierung der Diagonalelemente nicht eindeutig vorgegeben.

Stull und Driedonks [42] haben eine Methode vorgeschlagen, die den Übergang vom Mischungspotential zur Transilienzmatrix mit der Lösung dieses Problems verbindet. Diese Methode soll zunächst für ein äquidistantes Modellgitter dargestellt werden. Zuerst werden die Koeffizienten für das dimensionslose Mischungspotential für alle Nichtdiagonalelemente entsprechend Gl. 3.21 berechnet. Um einen Anhaltspunkt für die Berechnung der Diagonalelemente zu erhalten, werden Ergebnisse von Beobachtungen der atmosphärischen Grenzschicht und aus Laborexperimenten herangezogen. Diese zeigen u.a., daß auch in Situationen mit sehr starker thermischer Konvektion die Turbulenz zu einem Zustand einer gut durchmischten Grenzschicht führt, daß also neben den großskaligen thermisch induzierten Wirbeln auch die kleinerskaligen Wirbel gleichmäßig vertreten sind. Für das Mischungspotential heißt dies, daß alle Elemente, die den Austausch zwischen Schichten beschreiben, die innerhalb der turbulenten Grenzschicht liegen, besetzt sein müssen.

Eine Aussage über das Größenverhältnis der Diagonalelemente zu den übrigen Elementen liefert folgende Überlegung [2]. Es wird ein 2-Schichten-Modell mit transilienter Turbulenzschließung angenommen. Der turbulente Austausch sei 
so stark, daß die Luftmassen der Schichten vollständig ausgetauscht werden, die Diagonale der Transilienzmatrix folglich nicht besetzt ist. Stattdessen sei die Nebendiagonale besetzt und die Transilienzmatrix habe die folgende Gestalt:

$$
C=\left(\begin{array}{ll}
0 & 1 \\
1 & 0
\end{array}\right) .
$$

Eine passive skalare Größe habe in der unteren Schicht den Wert 1 und in der oberen Schicht den Wert 0. Die Werte dieser Größe nach einem Zeitschritt ergeben sich bei Quellen- und Senkenfreiheit durch Matrixmultiplikation zu:

$$
\left(\begin{array}{ll}
0 & 1 \\
1 & 0
\end{array}\right)\left(\begin{array}{l}
0 \\
1
\end{array}\right)=\left(\begin{array}{l}
1 \\
0
\end{array}\right) \text {. }
$$

Das entspricht einer vollständigen Umkehrung der Verhältnisse in vertikaler Richtung, was im englischen Sprachraum auch als "convective overturning" bezeichnet wird. In der realen Atmosphäre ist ein solches Verhalten nicht zu beobachten, sondern die Unterschiede zwischen den beiden Schichten würden beginnen, sich auszugleichen. Offensichtlich dürfen die Elemente der Nebendiagonalen nicht gegenüber den anderen Matrixelementen wertemäßig überwiegen.

Aus dieser Überlegung läßt sich für die Größenverteilung der Matrixelemente und damit auch für die Diagonalelemente folgendes ableiten [42]: Die Koeffizienten der Transilienzmatrix müssen sowohl zeilen- als auch spaltenweise zur Diagonale hin anwachsen und die Diagonalemente sind die wertemäßig größten Elemente in den jeweiligen Reihen und Spalten (diese Bedingung ist nicht sehr streng, so daß die der Diagonalen näher gelegene Elemente auch genauso groß wie weiter entfernte Elemente sein können). Diese Bedingung muß auch an das Mischungspotential gestellt werden.

Nach der Berechnung der Nichtdiagonalelemente werden bei diesem Verfahren zunächst alle Zeilen und Spalten überprüft, ob sie diese Bedingung erfüllen. Eventuell müssen Werte korrigiert und durch größenmäßig passende Werte ersetzt werden.

Um zu erreichen, daß die Elemente der Hauptdiagonalen die wertemäßig größten Koeffizienten in jeder Reihe der Matrix des dimensionslosen Mischungspotentials darstellen, werden Werte für $Y_{i i}$ zunächst nach

$$
Y_{i i}=\max \left(Y_{i, i-1}, Y_{i, i+1}\right)+Y_{\text {ref }}
$$

berechnet. Der noch hinzuaddierte Referenzwert $Y_{\text {ref }}$ für das dimensionslose Mischungspotential entlang der Hauptdiagonalen gewährleistet, daß $Y_{i i}$ immer größer ist, als die Elemente der Nebendiagonalen. Stull und Driedonks (1987) haben verschiedene Werte für den Parameter $Y_{\text {ref }}$ durch Vergleich der Simulationsergebnisse mit Messungen getestet. Ein Wert von $\approx 1000$ hat sich als richtige Größenordnung von $Y_{\text {ref }}$ herausgestellt. Für den Übergang zur Transilienzmatrix wird eine skalare Norm $\|Y\|$ definiert, die der maximalen Summe über die Matrixreihen des dimensionslosen Mischungspotentials entspricht:

$$
\|Y\|=\max _{i}\left(\sum_{j} Y_{i j}\right) .
$$


Die Nichtdiagonalelemente der Transilienzmatrix ergeben sich dann durch Normierung als:

$$
c_{i j}=\frac{Y_{i j}}{\|Y\|} \quad \text { für } \quad \mathrm{i} \neq \mathrm{j} .
$$

Erst jetzt können die Diagonalelemente berechnet werden nach:

$$
c_{i i}=1-\sum_{\substack{j=1 \\ j \neq i}}^{N} c_{i j} .
$$

Mit dieser Vorgehensweise wird erreicht, daß die Summe der Reihen und Spalten der Transilienzmatrix wie gewünscht gleich 1 ist, andererseits aber die Diagonalelemente die wertemäßig größten Elemente jeder Matrixreihe darstellen. Für ein nichtäquidistantes Modellgitter muß diese Methode zur Berechnung der Diagonalelemente noch modifiziert werden. In diesem Falle muß die skalare Norm $\|Y\|$ auch die unterschiedlichen Luftmassen der Modellschichten berücksichtigen, welche wieder durch die Schichtdicken ersetzt werden können:

$$
\|Y\|=\max _{i}\left(\sum_{j} h_{j} Y_{i j}\right) .
$$

Auch die Berechnung der Nichtdiagonalelemente der Transilienzmatrix muß entsprechend geändert werden:

$$
c_{i i}=1-\sum_{\substack{j=1 \\ j \neq i}}^{N} h_{j} c_{i j} .
$$

Stull hat 1993 eine verbesserte Methode zur Bestimmung der Diagonalelemente der Transilienzmatrix vorgestellt [41]. Diese Methode wurde auch im Falle dieser Arbeit angewendet. Dazu werden nach der Definition der Nichtdiagonalelemente nach Gl. 3.21 die Elemente des Mischungspotentials entlang der Hauptdiagonalen auf einen empirischen Referenzwert $Y_{\text {ref } f}$ gesetzt. Je größer der Wert von $Y_{\text {ref }}$, desto weniger Luft wird zwischen verschiedenen Schichten ausgetauscht. Der Übergang zur Transilienzmatrix erfolgt in einer separaten Iteration (siehe Abschnitt 3.4).

Das so definierte Mischungspotential liefert eine Aussage darüber, wie stark die Durchmischung zwischen verschiedenen Luftschichten sein würde, wenn man unter Ausschluß aller äußeren Quellen und Senken für die Turbulenzenergie (d.h. aller Effekte, die nicht in der Gleichung für die lokale zeitliche Änderung der Turbulenzenergie auftauchen, wie z.B. horizontale Advektion der Turbulenzenergie) so lange warten würde, bis die Turbulenz abgeklungen ist. Da in einem Modell jedoch häufig eine feinere zeitliche Auflösung erwünscht ist, muß die Matrix des Mischungspotentials noch entsprechend dem Modellzeitschritt modifiziert werden. Die so erhaltene Matrix wollen wir als Mischungsmatrix bezeichnen. 


\subsection{Abhängigkeit der Mischungsmatrix vom Modell- zeitschritt und Massennormierung}

Einen ersten Überblick über die Struktur von Transilienzmatrizen, die den turbulenten Austausch in der atmosphärischen Grenzschicht beschreiben, haben Ebert, Schumann und Stull [11] gegeben, indem sie Large-Eddy-Simulationen (LES) für eine konvektive Grenzschicht ohne Waldbestand durchführten und aus Modellexperimenten Transilienzmatrizen ableiteten. Genutzt wurde dabei das von Schumann et al. 1987 entwickelte MESOSCOP LES-Modell.

Ein Large-Eddy-Simulationsmodell löst die Navier-Stokes'schen Bewegungsgleichungen durch direkte Integration in einem sehr feinen dreidimensionalen Modellgitter und löst so die für den Turbulenzprozeß wichtigen Bewegungsskalen explizit auf. Somit kann das gesamte Spektrum von Wirbelgrößen berücksichtigt werden, also auch nichtlokale Prozesse. Es bietet damit eine gegenüber großangelegten Meßkampagnen relativ einfache Möglichkeit, das Wissen über die nichtlokalen Eigenschaften der atmosphärischen Turbulenz in einem Maße zu erweitern, wie es mittels Messungen nicht möglich wäre. Trotzdem ist die LES keine perfekte Simulationsmethode, da auch hier subgridskalige Prozesse approximiert werden müssen. Außerdem setzt der hohe Rechenaufwand der breiten Anwendung der LES Grenzen (siehe Abschn. 3.1, S. 17).

In der nun folgenden Beschreibung der Vorgehensweise zur Bestimmung von Transilienzmatrizen aus Modellergebnissen von Large-Eddy-Simulationen muß zwischen dem modellinternen Zeitschritt des LES-Modells (im folgenden „Modellzeitschritt" ) und dem Zeitschritt (der Zeitdauer), für den (die) die Transilienzmatrix gültig ist, unterschieden werden. Für letzteren soll im folgenden einfach die Bezeichnung "Zeitschritt" verwendet werden, da diese auch in allen anderen Kapiteln den Zeitschritt des transilienten Modells und damit der Transilienzmatrix bezeichnet.

Bei den von Ebert, Schumann und Stull durchgeführten LES wurde eine verschwindende mittlere Horizontalbewegung in der gesamten Grenzschicht angenommen, um Advektionserscheinungen auszuschließen. Die Ableitung von Transilienzmatrizen wurde folgendermaßen durchgeführt [11]:

- Zunächst wurde die LES bis zum Erreichen eines Zustandes quasi-stationärer Turbulenz ausgeführt und dann unterbrochen.

- Dann wurde jede Gitterbox in jeder Modellschicht mit der gleichen Menge an Teilchen von Tracern „,beladen“ . Dabei wurde jeder Schicht ein anderer Tracer zugeordnet. Damit ist also jeder Tracer ein Marker für die Herkunft eines Teilchens.

- Nach einem festgelegten Zeitpunkt, welcher dem gewünschten Zeitschritt entsprach, wurde festgestellt, inwieweit sich jeder Tracer bis in andere Gitterboxen ausgebreitet hat.

- Aus den Ergebnissen konnten durch horizontale Mittelung der Teilchenzahlen jedes Tracers in jeder Modellschicht auf einfache Weise Transilienzmatrizen gewonnen werden.

Dabei hat sich gezeigt, daß der Aufbau der Matrix erwartungsgemäß vom Zeit- 
schritt abhängt. Für sehr kurze Zeitschritte $(\Delta t \rightarrow 0)$ nähert sich die Struktur einer Einheitsmatrix [I], während sich für sehr große Zeitschritte $\left(\Delta t \rightarrow 4 t_{*}, t_{*}\right.$ ist der konvektive Zeitscale nach Deardorff [6]), das Mischungspotential einer Matrix für den gut durchmischten Zustand annähert. Die Matrixelemente sind dann innerhalb des turbulenten Bereiches von derselben Größe. Außerhalb des turbulenten Bereiches sind alle Matrixelemente gleich Null.

Die mittels LES gewonnenen Ergebnisse bezüglich der Zeitschrittabhängigkeit der Transilienzmatrizen gelten prinzipiell für alle turbulenten Grenzschichten, wenn horizontale Homogenität der Turbulenz angenommen werden kann.

Da die angegebene Parametrisierung des Mischungspotentials nicht explizit vom Zeitschritt abhängt, muß die Reaktion des Mischungspotentials auf unterschiedliche Zeitschritte parametrisiert werden. Nach Stull [41] kann man die Wurzel bzw. Potenz der Matrix für den Zeitschritt $t_{*}$ berechnen, um das Mischungspotential für den Zeitschritt $\Delta t$ zu erhalten:

$$
[Y(\Delta t)]=\left[Y\left(t_{*}\right)\right]^{N}
$$

mit $\mathrm{N}=\frac{\Delta t}{t_{*}}$. Diese Gleichung ist genauso für die Transilienzmatrix gültig, da die Iteration zur Luftmassen- und Konzentrationserhaltung die Struktur der Matrix nicht verändert (siehe Kap. 3.4, S. 38).

Diese Beziehung gilt jedoch nur unter der Voraussetzung, daß sich die Struktur der Turbulenz, die von der Transilienzmatrix beschrieben wird, während eines Zeitschrittes nicht ändert (quasi-stationäre Turbulenz). Bei einem nicht zu groß gewählten Zeitschritt ( $\leq 10 \mathrm{~min}$ ) kann diese Voraussetzung in den meisten Fällen als erfüllt betrachtet werden. Um beliebige nicht ganzzahlige Potenzen (auch Exponenten $<1$ ) von Matrizen zu bilden wird in der linearen Algebra als direktes Verfahren die Eigenwert-Eigenvektor-Methode angewendet. Dazu müssen sämtliche Eigenvektoren und Eigenwerte der Matrix bestimmt werden. Die allgemeine Eigenwertaufgabe besteht darin, Zahlen $\lambda_{i}$ (die Eigenwerte) zu finden, so daß die Beziehungen $A \vec{e}_{i}=\lambda_{i} \vec{e}_{i}$ für i $=1 \rightarrow 3$ erfüllt sind. Das gilt nur für bestimmte Spaltenvektoren $\vec{e}_{i}$, die Eigenvektoren der Matrix A. Die Eigenwerte und Eigenvektoren können komplex oder reell sein und sind einander zugeordnet. Jede nicht singuläre quadratische Matrix der Dimension N besitzt N Eigenwerte und N Eigenvektoren. Die Bildung einer beliebigen Potenz einer Matrix läßt sich allgemein für eine quadratische Matrix A folgendermaßen beschreiben:

Bildet man aus den Eigenwerten eine Diagonalmatrix L, indem jeder Eigenwert ein Diagonalelement darstellt und aus den Eigenvektoren eine Matrix E, wobei jeder Eigenvektor eine Spalte dieser Matrix darstellt, so gilt folgende Beziehung:

$$
Y=E L E^{-1} \text {. }
$$

Eine p-te Potenz von $Y$ läßt sich dann berechnen nach:

$$
Y=E L^{p} E^{-1} \text {. }
$$

Da L eine Diagonalmatrix ist, müssen also nur die $p$-ten Potenzen der Eigenwerte gebildet werden. Obwohl dieses Verfahren in seinen Grundzügen sehr einfach erscheint, wird es doch durch die notwendige Bestimmung der Eigenwerte und Eigenvektoren sehr aufwendig. Auch die Rechenzeit kann erheblich zunehmen. 
Aus diesen Gründen wurde von Inclán et al. [18] auf halbempirischem Wege eine algebraische Methode entwickelt, bei der sie eine dimensionslose Mischungsmatrix folgendermaßen definieren.

$$
\left(Y_{n, i j}\right)=\frac{1}{1+\left\{\frac{1}{\beta}\left[\frac{t_{n}}{\Delta t}\right]^{2}\right\}} \quad \text { für } \mathrm{i} \neq \mathrm{j} .
$$

Dabei ist $t_{n}$ die benötigte Transportzeit eines Luftpaketes von der Schicht $\mathrm{j}$ in die Schicht $\mathrm{i}$ und $\beta$ ist ein dimensionsloser Parameter. $\left(Y_{n, i j}\right)$ ist dann die zeitschritt-angepasste Mischungsmatrix. Im Verhältnis $\frac{t_{n}}{\Delta t}$ wird der Umstand berücksichtigt, daß nicht alle Luftpakete ihr eigentliches Ziel erreichen können, wenn ihnen nur die Zeit $\Delta t<t_{n}$ zur Verfügung steht. So soll die Strukturänderung der späteren Transilienzmatrix bei Verwendung unterschiedlicher Zeitschritte berücksichtigt werden (Zeitschrittadaptation). Jetzt muß eine Parametrisierung für die Zeit $t_{n}$ gefunden werden. Da die Definition des Mischungspotentials auf der turbulenten kinetischen Energie beruht, kann man eine represäntative turbulente Geschwindigkeit zwischen den Quell- und den Zielschichten als proportional zu $\sqrt{Y_{i j}}$ annehmen. Der Proportionalitätsfaktor geht in den Parameter $\beta$ ein. Da zwischen der Quell- und Zielschicht der Weg $\Delta z_{i j}$ zurückgelegt werden muß, kann $t_{n}$ berechnet werden als $\frac{\Delta z_{i j}}{\sqrt{Y_{i j}}}$ und man erhält für die Mischungsmatrix:

$$
\left(Y_{n, i j}\right)=\frac{Y_{i j}}{Y_{i j}+\frac{1}{\beta} \frac{\Delta z_{i j}^{2}}{\Delta t^{2}}} \quad \text { für } \mathrm{i} \neq \mathrm{j} .
$$

Der Parameter $\beta$ wurde aus Vergleichen mit Large-Eddy-Simulationen [11] abgeleitet. Der Wert von $\beta$ liegt im Bereich von $10^{-6}-10^{-4}$.

Führt man Grenzwertüberlegungen für $\Delta t$ durch, so findet man das für die Transilienzmatrix gewünschte Verhalten:

- Für den Fall $\Delta t \rightarrow 0$ streben die Nichtdiagonalelemente der Mischungsmatrix gegen 0 . Da aber die Summen der Reihenelemente der späteren Transilienzmatrix gleich 1 sein müssen, müssen die Diagonalelemente der Transilienzmatrix wie gewünscht gegen 1 streben (vergleiche Iteration nach Petterson Kap. 3.4, S. 38).

- Für den Fall $\Delta t \rightarrow \infty$ streben die Nichtdiagonalelemente der Mischungsmatrix gegen 1 , solange $Y_{i j} \neq 0$, sie sind sonst gleich 0 . Da aber die Summen der Reihenelemente der späteren Transilienzmatrix gleich 1 sein müssen, ergibt sich für diesen Fall, daß alle von 0 verschiedenen Elemente der Transilienzmatrix, die den turbulenten Bereich des Modellgebietes beschreiben, ungefähr von derselben Größe sein müssen. Im Vergleich mit den "gemessenen“ Transilienzmatrizen ebenfalls ein erwünschter Effekt.

Die bisherige Mischungsmatrix läßt sich nur in einem Modell mit äquidistanten Modellschichten verwenden. Für nichtäquidistante Schichten müssen relative Luftmassen eingeführt werden, mit denen die einzelnen Elemente der Mischungsmatrix multipliziert werden (siehe Abschnitt 3.1). Dadurch wird die Einhaltung der Kontinuitätsgleichung gewährleistet. Als gute Näherung für die relativen Luftmassen bietet sich das Verhältnis der Höhe der jeweiligen 
Quellschicht $h_{j}$ zur dünnsten Modellschicht $h_{\min }$ (meist die unterste Schicht) an. Die so veränderte Mischungsmatrix $\left(Y_{\omega, i j}\right)$ ergibt sich also mittels der Beziehung:

$$
\left(Y_{\omega, i j}\right)=\left(Y_{n, i j} \frac{h_{j}}{h_{\min }}\right)
$$

und wird als massennormierte Mischungsmatrix bezeichnet. Die Berücksichtigung der unterschiedlichen Dicke der Modellschichten bildet den letzten Schritt vor dem Übergang zur Transilienzmatrix durch Iteration.

\section{4 Übergang zur Transilienzmatrix}

Die Mischungsmatrix allein enthält zwar die für die mathematische Beschreibung des turbulenten Austauschprozesses wichtigen Größen, kann aber so noch nicht verwendet werden, da die 2 wichtigsten Forderungen - Luftmassenerhaltung und Konzentrationserhaltung im Modellgebiet - noch nicht erfüllt sind (siehe Kap. 3.1, S. 17). An ein Verfahren zur Umwandlung der Matrix des Mischungspotentials in eine entsprechende Transilienzmatrix mit Berücksichtigung von Massen- und Konzentrationserhalt ist die Forderung zu stellen, daß die relative Größenverteilung der Matrixelemente und damit die Struktur des turbulenten Austauschprozesses erhalten bleibt. Eine entsprechende Iteration wurde von J. Petterson vom IBM Bergen Scientific Center vorgeschlagen und von Inclán et al. erstmals angewendet [18]. Nennt man die durch die Iteration veränderte Mischungsmatrix $\left(Y_{n, i j}^{*}\right)$, dann läßt sich diese Iteration in 4 Schritte einteilen:

- 1. Schritt: Berechnung einer Matrix $\left(c_{i j}^{\text {row }}\right)$, die sich aus den Elementen von $\left(Y_{\omega, i j}^{*}\right)$ ergibt, indem jeweils durch die Summe der Elemente der entsprechenden Spalte von $\left(Y_{\omega, i j}^{*}\right)$ geteilt wird:

$$
c_{i j}^{r o w}=\frac{Y_{\omega, i j}^{*}}{\sum_{j=1}^{N} Y_{\omega, i j}^{*}}
$$

Die Summe der Elemente der Spalten dieser Matrix ist zu 1 normiert, was nicht für die Summe der Reihenelemente (unter Verwendung von Gleichung 3.4) der Fall sein muß.

- 2. Schritt: Berechnung einer Matrix $\left(c_{i, j}^{c o l}\right)$, die sich aus den Elementen von $\left(Y_{\omega, i j}^{*}\right)$ ergibt, indem jeweils durch die Summe der nach Gl. 3.4 mit dem Verhältnis der Luftmassen von Ziel- bzw. Quellschicht multiplizierten Elemente der entsprechenden Reihe von $\left(Y_{\omega, i j}^{*}\right)$ dividiert wird:

$$
c_{i j}^{c o l}=\frac{Y_{\omega, i j}^{*}}{\sum_{i=1}^{N} Y_{\omega, i j}^{*} \frac{m_{i}}{m_{j}}}
$$

Die Summe der Elemente der Reihen dieser Matrix ist (unter Verwendung von Gleichung 3.4) zu 1 normiert, was nicht für die Summe der Spaltenelemente der Fall sein muß. 
- 3. Schritt:Eine neue Matrix $\left(Y_{\omega, i j}^{*}\right)$ wird berechnet als Summe der beiden Matrizen $\left(c_{i j}^{r o w}\right)$ und $\left(c_{i j}^{c o l}\right)$ :

$$
Y_{\omega, i j}^{*}=\frac{c_{i j}^{\text {row }}+c_{i j}^{c o l}}{2} .
$$

- 4. Schritt: Test, ob die Summe der Reihen- bzw. Spaltenelemente der neuen Matrix bis auf einen kleinen Fehler (z.B. $10^{-4}$ ) gleich 1 ist, wobei bei den Reihensummen wiederum Gl. 3.4 verwendet werden muß. Ist dies nicht der Fall, erfolgt eine erneute Iteration, ansonsten ist die Transilienzmatrix [im folgenden: $\left.\left(C_{i j}\right)\right]$ gefunden.

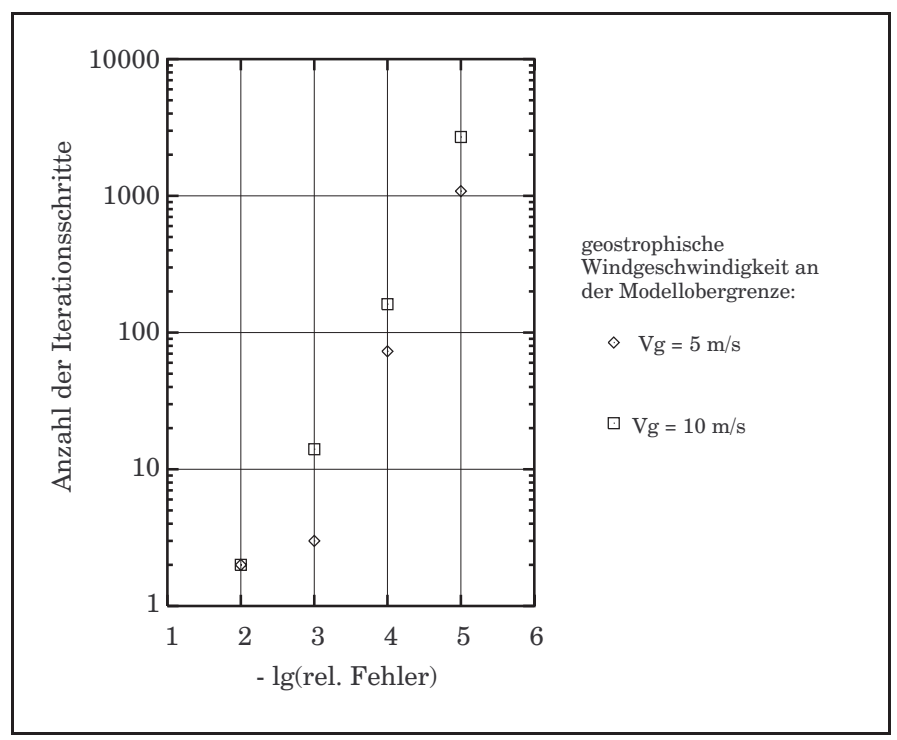

Abbildung 3.4: Anzahl der benötigten Iterationsschritte zur Berechnung der Transilienzmatrix aus der Mischungsmatrix als Funktion der gewünschten Genauigkeit für 2 spezielle atmosphärische Zustände (Erläuterung im Text)

Die Anzahl der durchzuführenden Iterationen hängt stark von der gewünschten Genauigkeit ab. Um einen Anhaltspunkt für die sinnvolle Wahl des Abbruchkriteriums für die Iteration zu bekommen, wurden einige Testrechnungen über jeweils einen Rechenzeitschritt von 3 min durchgeführt. Bei diesen Testrechnungen für eine Atmosphäre mit Waldbestand, zeigte sich zudem noch eine Abhängigkeit der Anzahl der Iterationen von der thermischen Schichtung und der vertikalen Änderung des Betrages der horizontalen Windgeschwindigkeit im Modellgebiet. Die Abbildung 3.4 zeigt für 2 spezielle Fälle die Anzahl der benötigten Iterationen zur Berechnung der Transilienzmatrix aus der Mischungsmatrix in Abhängigkeit von der gewünschten Genauigkeit. Auf der Abszisse aufgetragen ist der negative dekadische Logarithmus des in Kauf genommenen Fehlers (= maximale relative Abweichung der einzelnen Reihen- bzw. Spaltensummen der Elemente der Transilienzmatrix von 1), so daß der Fehler nach rechts abnimmt. Gerechnet wurde für eine im gesamten Modellgebiet thermisch neutral geschichtete Atmosphäre (auch im Bestand wurde der Einfachheit halber neutrale Schichtung angenommen). Das Profil 
der Windgeschwindigkeit wurde über dem Bestand als logarithmisch angenommen und möge in $3000 \mathrm{~m}$ Höhe mit der geostrophischen Windgeschwindigkeit $\left(V_{g}\right)$ zusammenfallen. Zur Berechnung des Windprofiles über dem Bestand wurden die aus Messungen bestimmten Werte der Verschiebungshöhe $d \approx 23 \mathrm{~m}$ und der Rauhigkeitslänge $\left(z_{0}=2.5 \mathrm{~m}\right)$ verwendet. Die Werte der Windgeschwindigkeit im Bestand wurden unter Verwendung einer theoretischen Formel nach Inoue und Cionco (entnommen aus [21]) berechnet:

$$
v=v_{p} \exp \left[-\alpha\left(1-\frac{z}{h_{p}}\right)\right] ; \alpha=h_{p} \sqrt[3]{0.5 c_{d} A_{\text {tree }}(z) \bar{l}^{2}},
$$

mit: $v_{p} \quad-\quad$ Windgeschwindigkeit an der Bestandsobergrenze

$z \quad$ - Höhe im Bestand

$h_{p} \quad$ - Höhe der Bestandsobergrenze

$c_{d} \quad$ - aerodyn. Widerstandskoeffizient des Bestandes

$A_{\text {tree }}$ - Projektion der Vegetationsoberfläche in Windrichtung

$\bar{l} \quad$ - mittlere Mischungsweglänge im Bestand.

Die angenommenen 2 Fälle unterscheiden sich nur im Wert der geostrophischen Windgeschwindigkeit ( 5 und $10 \mathrm{~m} / \mathrm{s}$ ) an der Obergrenze des Simulationsgebietes. Aus der graphischen Darstellung läßt sich eine starke Abhängigkeit der nötigen Iterationschritte von der geforderten Genauigkeit ablesen, während die Abhängigkeit von der Struktur des Windprofiles geringer ist. Als Kompromiß zwischen der Genauigkeit mit der die Luftmassen- und Konzentrationserhaltung im Modellgebiet gewährleistet ist, und der Effizienz der Rechengeschwindigkeit wurde in allen folgenden Modellsimulationen mit einer rel. Genauigkeit von $10^{-4}$ gerechnet.

Die Schritte zur Berechnung der Transilienzmatrix sind in der Abbildung 3.5 nochmals zusammenfassend dargestellt.

\subsection{Struktur der Transilienzmatrix}

Was kann aus der Struktur der Transilienzmatrix abgelesen werden? Als Beispiel einer Transilienzmatrix soll Abb. 3.6 dienen - eine Isoliniendarstellung einer Transilienzmatrix. Die Darstellung dieser Matrix weicht von der mathematischen Definition der Zeilen einer Matrix ab, und zwar werden die Zeilennummern entsprechend der meteorologischen z-Koordinate von unten an gezählt, so daß die Matrix praktisch an der Horizontalen gespiegelt wird. Dadurch stellt die Nebendiagonale in der neuen Darstellungsform die Hauptdiagonale der ursprünglichen Matrix dar. Alle weiteren Transilienzmatrizen in dieser Arbeit sind in derselben Form dargestellt. Mit dem Begriff Diagonale ist im folgenden immer die Hauptdiagonale gemeint, wenn es nicht anders angegeben ist.

Um Kommastellen bei den Isolinienbezeichnungen zu vermeiden, wurden alle Elemente der Transilienzmatrix mit 1000 multipliziert. Die Isoliniendarstellung zeigt also, wieviel Promille (nicht Prozent !) der Luftmasse einer Schicht aus einer anderen Schicht herantransportiert wird. $\mathrm{Zu}$ beachten ist auch die nichtlineare Zuordnung zwischen Modellschichtnummer und -höhe, die sich 


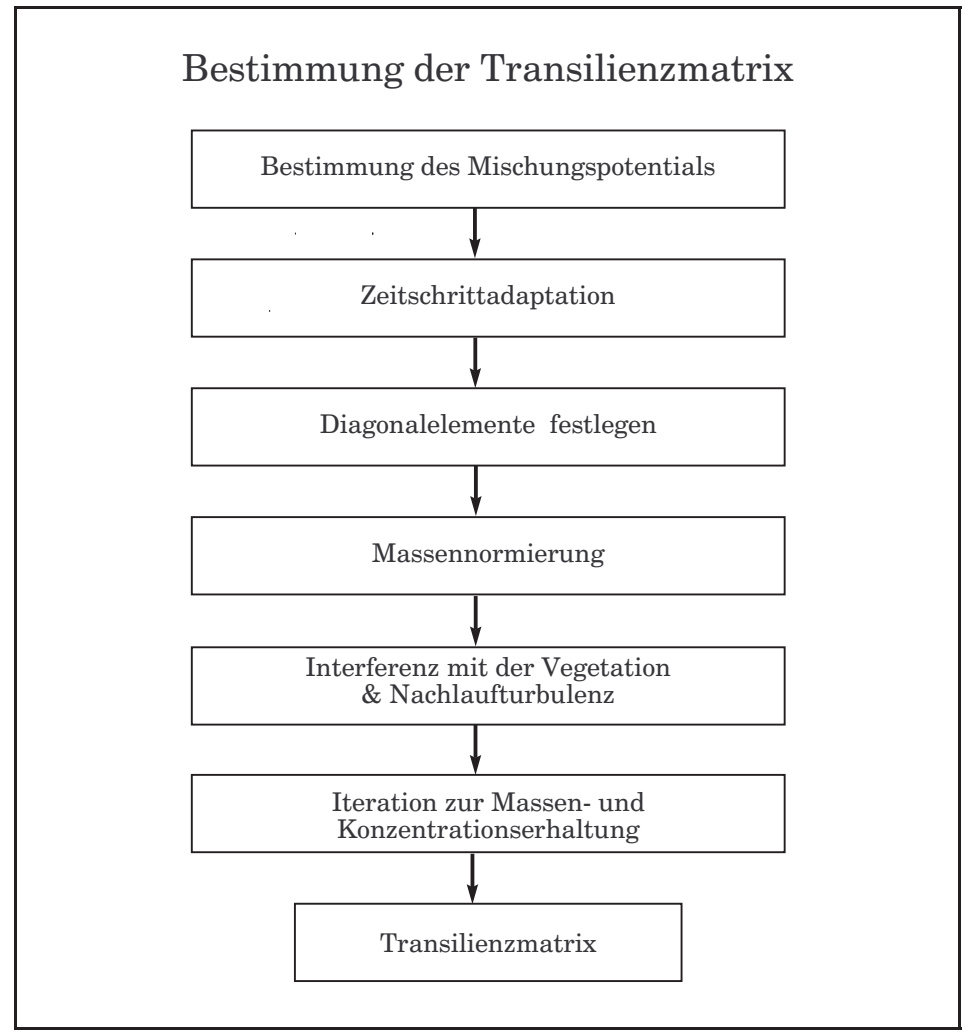

Abbildung 3.5: Schritte bei der Berechnung der Transilienzmatrix

aus der nichtäquidistanten Schichteinteilung des Modells ergibt (siehe Kap. 5) !

Betrachten wir einen beliebigen Punkt innerhalb der Matrix, so bezeichnet die Abszisse den Herkunftsort, also die Quellschicht eines Luftpaketes, während die Ordinate den Bestimmungsort, also die Zielschicht bezeichnet. Verwendet man als Koordinaten die Schichtnummern und liegt z.B. der Punkt $(33,26)$ auf der Isolinie 50, so heißt dies, daß 50 Promille der Luftmasse der Schicht 26 aus der Schicht 33 herantransportiert werden. In der Beispielmatrix ist zusätzlich noch der Bereich des Kronenraumes (Schichten 16 bis 26) durch horizontale bzw. vertikale Linien gekennzeichnet.

Die Elemente auf der Diagonalen kennzeichnen den Anteil der Luftmasse einer Schicht, welcher in dieser Schicht verbleibt. Die Elemente beiderseits der Diagonale (siehe auch die grau unterlegten Felder in Abb. 3.1 auf Seite 21) kennzeichnen den lokalen Austausch, also den Austausch zwischen benachbarten Schichten. Alle anderen Elemente bezeichnen den nichtlokalen Austausch.

Je größer die Differenz zwischen Quell- und Zielindex, je weiter ein Punkt also von der Diagonale entfernt ist, desto nichtlokaler ist der durch ihn repräsentierte Austausch, desto größer ist aber auch die Geschwindigkeit, mit der der Transport geschieht, da die Matrix alle die Transporte beschreibt, die innerhalb des entsprechenden Zeitschrittes stattfinden. Die Matrix ermöglicht also 


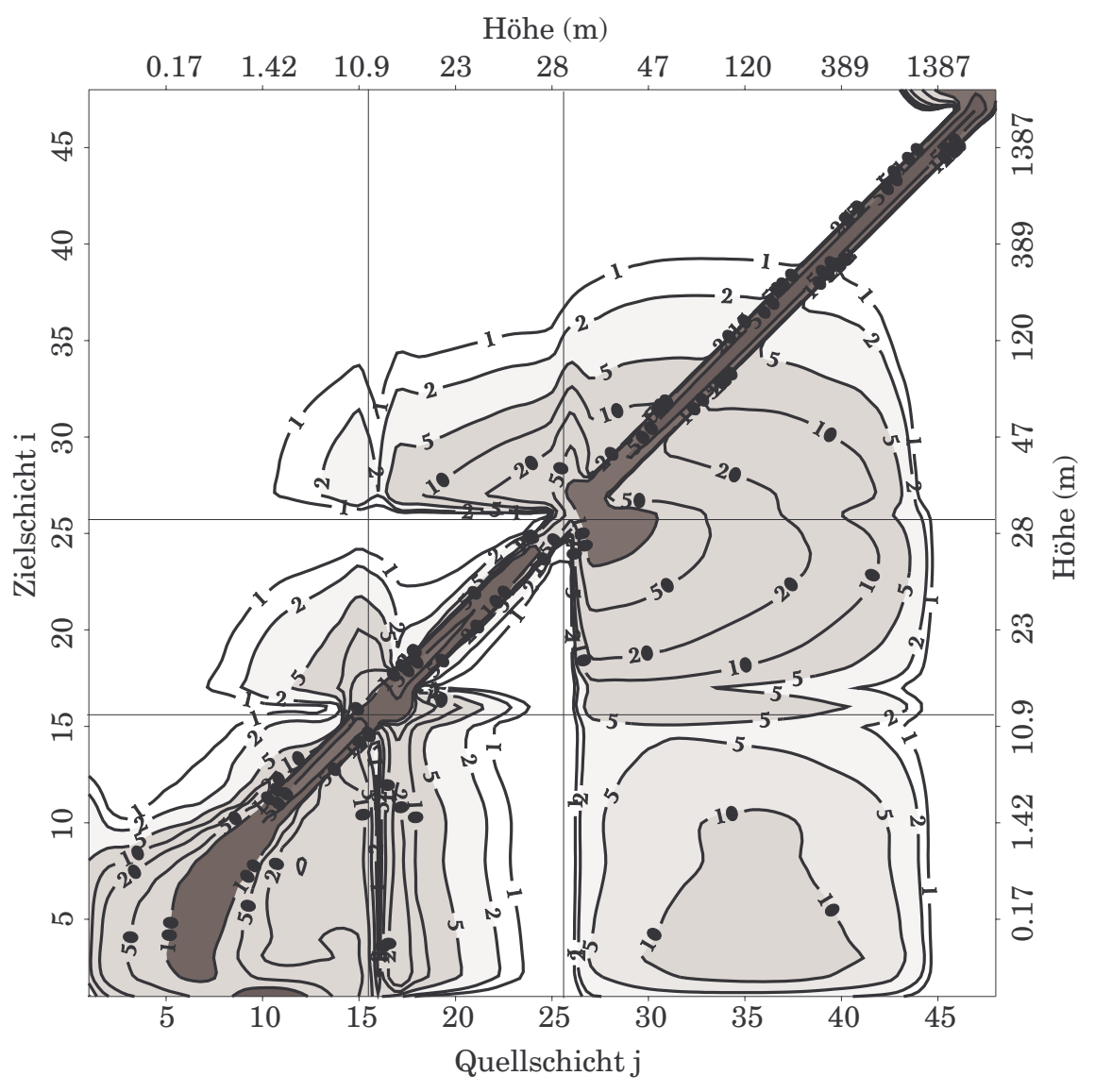

Abbildung 3.6: Beispiel einer Transilienzmatrix des verwendeten Grenzschichtmodells für einen Zeitschritt von $10 \mathrm{~min}$

auch Aussagen über die auftretenden Transportgeschwindigkeiten. Das soll die Abb. 3.7 verdeutlichen. Welche Transportgeschwindigkeit einem Matrixelement zugeschrieben werden kann, hängt allein vom Abstand dieses Matrixelementes senkrecht zur Diagonale ab. Die Diagonalelemente beschreiben den subskaligen Austausch, geben also an, wieviel der Luftmasse einer Schicht in dieser verbleibt. Ihnen kann allein mittels der Transilienzmatrix keine Transportgeschwindigkeit zugeordnet werden. Die der Diagonale unmittelbar benachbarten Elemente beschreiben den langsamen auf- bzw. abwärtsgerichteten Transport durch Wirbel mit kleinem Durchmesser. Langsam deshalb, da sie in dem der Matrix entsprechenden Zeitschritt nur den Transport zwischen Quell - und Zielschichten mit geringem Abstand zueinander beschreiben. Dagegen beschreiben die Elemente in den Ecken "oben links" und "unten rechts" der Matrix den sehr schnellen Austausch durch Wirbel mit großem Durchmesser. 


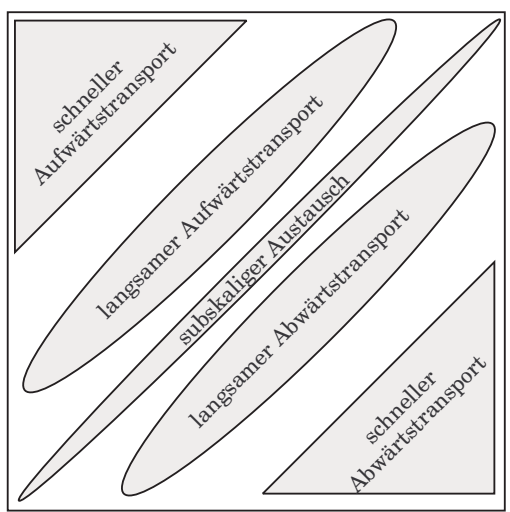

Abbildung 3.7: Zuordnung von Transportrichtung und -geschwindigkeit zu verschiedenen Gebieten der Transilienzmatrix nach Ebert, Schumann und Stull [11]

\subsection{Berechnung der turbulenten Flüsse}

Die transiliente Turbulenztheorie allein kann nur den turbulenten Anteil der auftretenden vertikalen Flüsse erklären, nicht jedoch den advektiven Anteil, der durch eine im zeitlichen Mittel nicht verschwindende Vertikalbewegung auftritt, oder die nichtturbulenten Anteile der Flüsse an festen Oberflächen (z.B. Erdoberfläche).

Nach Ni [28] ergibt sich für den aufwärtsgerichteten Fluß während des Zeitschrittes $\Delta t$ durch die Obergrenze der Modellschicht $\mathrm{k}$ mit der Dicke $h_{k}$ in eine Schicht i (die Zielschicht i liegt oberhalb des Niveaus k, alle Quellschichten j unterhalb) die folgende Beziehung:

$$
\frac{1}{\Delta t} \sum_{j=1}^{k} c_{i j}(t, \Delta t) S_{j}(t) h_{i} \quad \text { für } \mathrm{k}+1 \leq \mathrm{i} \leq \mathrm{N} .
$$

Entsprechend ergibt sich für den abwärtsgerichteten Fluß durch die Obergrenze der Schicht $\mathrm{k}$ in eine Schicht $\mathrm{i}$ (die Zielschicht $\mathrm{i}$ liegt unterhalb des Niveaus $k, \ldots)$ :

$$
\frac{1}{\Delta t} \sum_{j=k+1}^{N} c_{i j}(t, \Delta t) S_{j}(t) h_{i} \quad \text { für } 1 \leq \mathrm{i} \leq \mathrm{k} .
$$

Damit ergibt sich für den Gesamtfluß durch die Obergrenze der Schicht k (abwärtsgerichtete Flüsse erhalten per Konvention ein negatives Vorzeichen):

$$
F_{k}(t, \Delta t)=\frac{1}{\Delta t}\left[\sum_{i=k+1}^{N} \sum_{j=1}^{k} c_{i j}(t, \Delta t) S_{j} h_{i}-\sum_{i=1}^{k} \sum_{j=k+1}^{N} c_{i j}(t, \Delta t) S_{j} h_{i}\right] .
$$

Zur weiteren Vereinfachung werden die Summationsindizes i und j beim ersten Term in der Klammer vertauscht und man erhält: 


$$
F_{k}(t, \Delta t)=\frac{1}{\Delta t} \sum_{i=1}^{k} \sum_{j=k+1}^{N}\left(c_{j i}(t, \Delta t) S_{i} h_{j}-c_{i j}(t, \Delta t) S_{j} h_{i}\right) .
$$

Es läßt sich nun zeigen, daß die folgende Doppelsumme gleich Null ist:

$$
\sum_{i=1}^{k} \sum_{j=1}^{k}\left(c_{j i}(t, \Delta t) S_{i} h_{j}-c_{i j}(t, \Delta t) S_{j} h_{i}\right) .
$$

Dazu vertauscht man in diesem Term die Indizes i und $\mathrm{j}$ und addiert den so neu erhaltenen Term hinzu. Die Summe beider Terme ergibt Null. Da aber beide denselben Wert haben, müssen beide Terme gleich Null sein. Unter Benutzung dieser Tatsache kann man die innere Summe in der Gleichung für $F_{k}$ erweitern, und man erhält für den Fluß $F_{k}$ die folgende Beziehung:

$$
F_{k}(t, \Delta t)=\frac{1}{\Delta t} \sum_{i=1}^{k} \sum_{j=1}^{N}\left(c_{j i}(t, \Delta t) S_{i} h_{j}-c_{i j}(t, \Delta t) S_{j} h_{i}\right) .
$$

Damit ergibt sich die folgende Rekursionsbeziehung:

$$
F_{k}(t, \Delta t)-F_{k-1}(t, \Delta t)=\frac{1}{\Delta t} \sum_{j=1}^{N}\left[c_{j k}(t, \Delta t) S_{k} h_{j}-c_{k j}(t, \Delta t) S_{j} h_{k}\right] .
$$

Aufgrund der Luftmassen- und Konzentrationserhaltung im Modellgebiet läßt sich der erste Teil der Summe folgendermaßen umformen:

$$
\sum_{j=1}^{N} c_{j k}(t, \Delta t) S_{k} h_{j}=S_{k} \sum_{j=1}^{N} c_{j k}(t, \Delta t) h_{j}=S_{k} h_{k} .
$$

Dabei wird im zweiten Schritt von der Beziehung 3.4 und der Proportionalität zwischen der Luftmasse und der Höhe einer Schicht Gebrauch gemacht. Damit erhält man dann die folgende allgemeine Beziehung zur Berechnung der Flüsse bei transilienter Turbulenzschließung:

$$
F_{k}(t, \Delta t)=F_{k-1}(t, \Delta t)+\frac{h_{k}}{\Delta t} \sum_{j=1}^{N} c_{k j}(t, \Delta t)\left(S_{k}-S_{j}\right) .
$$

An der Bodenoberfläche gilt diese Beziehung nicht mehr, hier muß für $F_{k-1}$ der entsprechende Fluß zwischen dem Boden und der direkt darüberliegenden atmosphärischen Schicht $\left(F_{0}\right)$ eingesetzt werden.

\subsection{Mischungsintensität, Prozeßspektrum und Mi- schungsweglänge}

Die Transilienzmatrix enthält noch weitere Informationen insbesondere über Turbulenzparameter wie Mischungsintensität und Mischungsweglänge etc.. Zunächst soll hier das Transportspektrum interessieren. Im Gegensatz zu den 
Spektren, welche man mittels FFT (Fast Fourier Transformation) aus EddyKorrelationsmessungen gewinnt, handelt es sich hierbei um ein Spektrum, welches nicht den augenblicklichen Zustand, sondern den Prozeß des (nichtlokalen!) turbulenten Transportes beschreibt. Um Transportspektren auf der Grundlage der Transilienzmatrix berechnen zu können, muß auf die Gleichung zur Berechnung der turbulenten Flüsse zurückgegriffen werden. Eine Transportspektrum erhält man, wenn man nicht über alle Paare von Gitterpunkten des Modells summiert, sondern nur über diejenigen, welche einen bestimmten $\mathrm{Ab}$ stand zueinander haben, z.B. $H_{m}$ (mit $H_{m}=\left|h_{k}-h_{l}\right|$ und $\left.m=|k-l|\right)$ ). Den Abstand $H_{m}$ kann man auffassen als Größe einer Gruppe von Eddies, wobei im Transportspektrum der Anteil dieser bestimmten Eddies am Gesamtfluß der Eigenschaft $S$ zum Ausdruck kommt. Nach der Summation erhält man die folgende Beziehung:

$$
S_{T}^{k, m}=\frac{1}{\Delta t} \sum_{i=1}^{k} \sum_{j=k+1}^{N} \delta_{m,|i-j|}\left[c_{j i}(t, \Delta t) S_{i} h_{j}-c_{i j}(t, \Delta t) S_{j} h_{i}\right] .
$$

Durch Summation über die Transportspektren aller Wellenlängen erhält man wieder den Gesamtfluß. Es ist zu beachten, daß sich für ein und denselben Zeitpunkt, aber unterschiedliche Eigenschaften $S$ verschiedene Transportspektren ergeben können.

Es kann jedoch auch vorkommen, daß in einer bestimmten Höhe zwar sehr starke turbulente Durchmischung herrscht, aber der Beitrag zum Gesamtfluß nur sehr klein ist, da die Differenzen der betrachteten Größe zwischen zwei verschiedenen Höhen sehr klein sind. Wenn man eine Größe finden will, die nur den Prozeß der Turbulenz allein beschreibt, dann dürfen in deren Definition keine meteorologischen Größen auftreten. So wird das luftmassenbezogene Transportspektrum folgendermaßen definiert:

$$
S_{\text {Tair }}^{k, m}=\frac{1}{\Delta t} \sum_{i=1}^{k} \sum_{j=k+1}^{N} \delta_{m,|i-j|}\left[c_{j i}(t, \Delta t) h_{j}-c_{i j}(t, \Delta t) h_{i}\right] .
$$

Ersetzt man die Differenz durch eine Summe, so kommt dies der „Integration" über alle Wirbel gleich, die Luftpakete durch ein bestimmtes Höhenniveau aufwärts, aber auch abwärts transportieren. Somit gewinnt man eine Aussage über die Beiträge der einzelnen Wirbelgrößen zum gesamten turbulenten Massentransport an Luft in einem bestimmten Niveau der atmosphärischen Grenzschicht. Das so erhaltene Spektrum wird als Prozeßspektrum bezeichnet:

$$
S_{P}^{k, m}=\sum_{i=1}^{k} \sum_{j=k+1}^{N} \delta_{m,|i-j|} \cdot\left[c_{j i}(t, \Delta t)+c_{i j}(t, \Delta t)\right] .
$$

Die Summe über die Beiträge aller Eddy-Größen liefert die integrale Mischungsintensität:

$$
P_{k}(t, \Delta t)=\sum_{m=1}^{N-1} S_{P}^{k, m}(t, \Delta t)
$$

Nach Prandtl (1925) ergibt sich die Mischungsweglänge als die durchschnittliche Distanz, über welche die Luftpakete infolge der Turbulenz vertikal transportiert werden. Auch aus der Transilienzmatrix lassen sich der Prandtl'schen 
Mischungsweglänge ähnliche Größen bilden [41]. Betrachtet man jeweils nur die aufwärtsgerichteten Flüsse oder nur die abwärtsgerichteten Flüsse, so kann man 2 Arten von Mischungsweglängen bilden [11]. Es können so mittlere gerichtete Mischungswege von einem Quellniveau („Wegtransport“ ) und Mischungswege zu einem Zielniveau („Hintransport“ ) gebildet werden. Im ersteren Falle ergeben sich 2 Beziehungen für den "aufwärtsgerichteten“ Mischungsweg $l^{\uparrow}$ und für den ,abwärtsgerichteten“ Mischungsweg $l^{\downarrow}$ :

$$
l_{j}^{\dagger}=\frac{\sum_{i=j}^{N} c_{i j}(\Delta t) \frac{m_{i}}{m_{j}}\left|z_{i}-z_{j}\right|}{\sum_{i=j}^{N} c_{i j}(\Delta t) \frac{m_{i}}{m_{j}}}, \quad l_{j}^{\downarrow}=\frac{\sum_{i=1}^{j} c_{i j}(\Delta t) \frac{m_{i}}{m_{j}}\left|z_{i}-z_{j}\right|}{\sum_{i=1}^{j} c_{i j}(\Delta t) \frac{m_{i}}{m_{j}}},
$$

im letzteren Falle entsprechend:

$$
l_{i}^{\uparrow}=\frac{\sum_{j=1}^{i} c_{i j}(\Delta t)\left|z_{i}-z_{j}\right|}{\sum_{j=1}^{i} c_{i j}(\Delta t)}, \quad l_{i}^{\downarrow}=\frac{\sum_{j=i}^{N} c_{i j}(\Delta t)\left|z_{i}-z_{j}\right|}{\sum_{j=i}^{N} c_{i j}(\Delta t)} .
$$

Die Formeln entsprechen denen in der angegebenen Literatur, sie wurden nur für nichtäquidistante Modellschichten angepaßt. So mußte z.B. bei den ersten 2 Gleichungen mit dem Verhältnis der Luftmassen $\frac{m_{i}}{m_{j}}(\hat{=}$ dem Verhältnis der Schichtdicken) gewichtet werden (siehe 3.4, Seite 20). Die so berechneten Mischungsweglängen entsprechen einem mit den Koeffizienten der Transilienzmatrix gewichteten Integral über alle Wirbelgrößen.

Durch eine gewichtete Mittelung über die Beziehungen 3.54 und 3.55 unter Ausnutzung der Bedingungen für die Luftmassen- und Konzentrationserhaltung erhält man für jede Modellschicht k eine der Prandtl'schen Mischungsweglänge entsprechende Größe:

$$
l_{k}=\frac{\sum_{i=1}^{N}\left[c_{i k}(t, \Delta t) \frac{m_{i}}{m_{k}}+c_{k i}(t, \Delta t)\right]\left|z_{i}-z_{k}\right|}{2+2 c_{k k}} .
$$

Wichtig ist die Berücksichtigung der Diagonalelemente der Transilienzmatrix im Nenner der Gleichung. Die in der Arbeit von Ebert et al. 1989 angegebene Beziehung für $l_{k}$ berücksichtigt nicht die Besetzung der Diagonalelemente der Transilienzmatrix und ist deshalb nicht korrekt.

\subsection{Verwendung des transilienten Schließungsansat- zes in einem Grenzschichtmodell}

Um den transilienten Turbulenzschließungsansatz in einem atmosphärischen Grenzschichtmodell anwenden zu können, müssen auch noch die Quellen und Senken atmosphärischer Eigenschaftsgrößen berücksichtigt werden. Dazu muß jeder Modellzeitschritt in 2 Teile aufgesplittet werden [38]: 
- In einem ersten Teil werden die Profiländerungen durch die Eigenschaftsquellen und -senken berechnet und zum Profil der jeweiligen atmosphärischen Eigenschaft dazuaddiert. Dieser Teil kann als „Destabilisierung“ bezeichnet werden.

- Im zweiten Teil erfolgt dann die Berechnung der Transilienzmatrix und die Berechnung der neuen Profile auf der Grundlage der "destabilisierten“ Profile. Dieser Teil beschreibt die Reaktion der Atmosphäre auf die Änderungen durch Quellen und Senken.

Diese Methode kann mathematisch beschrieben werden, in dem man die Gleichung 3.1, S. 18 für jede atmosphärische Eigenschaft um einige Terme folgendermaßen erweitert:

$$
S_{i}(t+\Delta t)=\sum_{j=1}^{N} c_{i j}(t, \Delta t)\left[S_{j}(t)+Q_{j}(t)+S e_{j}(t)\right] .
$$

Dabei sind $Q_{j}(t)$ und $S e_{j}(t)$ die Quellen- bzw. Senkenterme der Eigenschaft $S$. Auch bei der Berechnung der turbulenten Eigenschaftsflüsse müssen erst die Randbedingungen sowie Quellen und Senken berücksichtigt werden, bevor die Flüsse mit den schon beschriebenen Gleichungen berechnet werden können. 


\section{Kapitel 4}

\section{Der Einfluß hoher Pflanzenbestände in der Turbulenzschließung}

\subsection{Formwiderstand}

Strömt die Luft durch einen Vegetationsbestand, so treten in Bezug auf die Strömung 2 Phänomene auf:

1. Es kommt zu einem Impulsverlust der Luft bezüglich der mittleren horizontalen Strömung. Dieser Impulsverlust kommt zustande durch den aerodynamischen Widerstand des Pflanzenbestandes.

2. Dieser Anteil an Impuls der mittleren Strömung wird größtenteils in Impuls der turbulenten Luftbewegung umgewandelt und dissipiert dann weiter bis zum vollständigen Zerfall der Turbulenzkörper und deren Umwandlung in Wärme. Aber auch die Vegetationselemente selbst nehmen kinetische Energie auf, was in deren Schwingungen Ausdruck kommt. Diese Schwingungsenergie dissipiert schließlich auch in Wärme oder wird von den Vegetationselementen wieder an die Luft abgegeben und dabei in kinetische Energie der turbulenten Luftbewegung umgewandelt. Die mit dem Schwingen der Vegetationselemente verbundenen Prozesse sind kompliziert und noch wenig untersucht und werden deshalb hier nicht weiter berücksichtigt. Außerdem ist anzunehmen, daß durch diese Vernachlässigung kein größerer Fehler verursacht wird, da der wichtige Parameter des aerodynamischen Widerstandes $c_{d}$ über Messungen bestimmt werden muß, wobei diese Prozesse implizit mit eingeschlossen werden.

In der allgemeinsten Form läßt sich die Änderung der Komponenten der horizontalen Windgeschwindigkeit $\Delta U_{k}=\Delta u_{k}+\Delta v_{k}$ in einer Modell-Schicht $\mathrm{k}$ 
(z.B. infolge von Reibung) wie folgt beschreiben:

$$
\begin{aligned}
& u_{k}(t+\Delta t)=u_{k}(t)-f\left(v_{g}-v_{k}(t)\right) \Delta t+\Delta u_{k} \\
& v_{k}(t+\Delta t)=v_{k}(t)+f\left(u_{g}-u_{k}(t)\right) \Delta t+\Delta v_{k},
\end{aligned}
$$

dabei sind:

$$
\begin{array}{lll}
u_{k} & - & \text { x-Komponente der Windgeschwindigkeit in der Schicht k } \\
v_{k} & - & \text { y-Komponente der Windgeschwindigkeit in der Schicht k } \\
u_{g}, v_{g} & - & \text { x- und y-Komponente der geostrophischen Windgeschw. } \\
\Delta u_{k} & - & \text { Änderung von } u_{k} \text { während des Zeitschrittes } \Delta t \\
\Delta v_{k} & - & \text { Änderung von } v_{k} \text { während des Zeitschrittes } \Delta t \\
\mathrm{f} & - & \text { Coriolisparameter } \\
\Delta t & - & \text { Modell-Zeitschritt. }
\end{array}
$$

Effekte der horizontale Advektion meteorologischer Größen werden aufgrund der Beschränkung des Modells auf eine Dimension (Vertikalkoordinate) vernachlässigt. Ebenfalls aufgrund der Beschränkung auf eine Dimension wird horizontale Homogenität des Bestandes, also auch der Struktur der Bäume, vorausgesetzt. Mit dem Begriff Baum ist deshalb immer der "Durchschnitts" -Baum bezogen auf den gesamten zu modellierenden realen Bestand gemeint.

Die Änderung der Windkomponenten erfolgt durch den aerodynamischen Widerstand der Blätter, Zweige, Äste und Stämme der Bäume. Der die Strömung primär verursachende Druck ist eigentlich eine Druckdifferenz welche dem horizontalen Luftdruckgradienten des Luftdruckes zwischen dem nächstgelegenen Bodenluftdruckmaximum und -minimum entspricht. Bei der Strömung durch einen Baumbestand wird dieser Druck ergänzt durch einen Druck, der durch den aerodynamischen Widerstand der Bäume und die Bodenreibung verursacht wird. Dieser Druck wirkt dem Druck infolge der Bodenluftdruckdifferenz entgegen und kann in Bezug auf diese als Druckverlust bezeichnet werden.

Die durch den aerodynamischen Widerstand eines Körpers verursachte Widerstandskraft $F_{w}$ bei der Umströmung durch eine horizontale atmosphärische Strömung läßt sich allgemein berechnen nach:

$$
F_{w}=c_{d} \rho A U^{2} .
$$

Dabei ist $c_{d}$ der aerodynamische Widerstandskoeffizient, $\rho$ die Luftdichte, $A$ die Querschnittsfläche des Körpers senkrecht zur Strömung und $U$ der Betrag der horizontalen Windgeschwindigkeit. Oft wird auch noch ein Vorfaktor 0.5 benutzt, der in diesem Falle aber schon bei der Bestimmung des aerodynamischen Widerstandskoeffizienten berücksichtigt wurde. Im Falle eines vertikal aufgelösten Schichtenmodells für einen Bestand, muß diese Kraft für jede Schicht k bestimmt werden. Dann müssen auch die horizontale Windgeschwindigkeit und die Querschnittsfläche der Vegetation senkrecht zur Anströmung der entsprechenden Schicht in die Gleichung eingesetzt werden. Für den aerodynamischen Widerstandskoeffizienten $c_{d}$ wird hier ein höhenkonstanter Wert verwendet, der auch nicht von der in einer Schicht enthaltenen Blattfläche abhängt. Zur Bestimmung von $c_{d}$ werden oft Windkanalmessungen durchgeführt, wobei meist die Gesamtkraft auf einen Einzelbaum gemessen wird, weshalb nach Gl. 4.3 nur ein einziger, damit höhenkonstanter Wert 
für einen Baum bestimmt werden kann. Außerdem wird in diesem Modell der Wert für $c_{d}$ als zeitlich konstant angenommen, $\mathrm{d}$. h. von der Windgeschwindigkeit unabhängig. Es gibt Messungen des aerodynamischen Widerstandes einzelner Vegetationselemente, welche eine Abhängigkeit von der Windgeschwindigkeit belegen. Ihre Übertragung auf einen ganzen Baum oder Bestand ist jedoch mit großen Unsicherheiten verbunden, da sich die aerodynamischen Widerstände der einzelnen Elemente nicht additiv verhalten ([27], S. 106ff). Kerzenmacher 1994 gibt vertikal aufgelöste Werte für den aerodynamischen Widerstand in einem Bestand an und erhält Werte von $c_{d}=0.2$ in den untersten $60 \%$ der Baumhöhe sowie $c_{d}=0.1$ für die oberen $40 \%$ der Baumhöhe. Allerdings wurden diese Werte nicht für den in dieser Arbeit untersuchten Bestand bestimmt und sind somit nicht ohne weiteres übertragbar. Mit zeitlich und vertikal konstanten Widerstandskoeffizienten konnten für Simulationen der Luftströmung durch Waldbestände der gemäßigten Breiten jedoch trotzdem gute Simulationsergebnisse erzielt werden (z.B. [46], [26]). Aus diesem Grunde wurde auch in dieser Arbeit ein konstanter aerodynamischer Widerstandskoeffizient verwendet. Der Wert von $c_{d}$ beträgt 0.2 .

Desweiteren wird bei der Bestimmung des aerodynamischen Widerstandes nach Gleichung 4.3 nicht die Oberfläche der Vegetationselemente senkrecht zur Anströmung verwendet, sondern die leichter bestimmbare horizontale Oberfläche. Diese kann aus Messungen der vertikalen Verteilung der kurzwelligen Strahlung im Bestand abgeleitet werden. Zwischen der vertikalen und der horizontalen Oberfläche bestehen Unterschiede, die aber im Wert des aerodynamischen Widerstandskoeffizienten $c_{d}$ aufgrund der Methode zu dessen Bestimmung berücksichtigt werden.

Für einen Bestand muß nun noch beachtet werden, daß zwischen den Bäumen je nach Bestandsdichte Lücken bestehen, in denen die Luftströmung auf keinen aerodynamischen Widerstand trifft. Bei bekannter Bestandsdichte wird deshalb die Fläche $A_{k}$ der Schicht $\mathrm{k}$ auf die gesamte jedem Baum in der Schicht k zur Verfügung stehende horizontale Fläche bezogen, $d$. h. es ergibt sich eine neue Fläche $A_{k}^{*}$ folgendermaßen:

$$
A_{k}^{*}=A_{k} \frac{\frac{\pi}{4} w_{t r e e, k}^{2}}{\Delta r^{2}} .
$$

Dabei ist $w_{t r e e, k}$ der horizontale Baum- (Kronen- , Stamm-) durchmesser in der Schicht $\mathrm{k}$ und $\Delta r$ ist die Seitenlänge der jedem Baum im Mittel zur Verfügung stehenden Bodenoberfläche in Ost-West bzw. Nord - Süd - Richtung. Wegen der Voraussetzung der horizontalen Homogenität des Bestandes, was auch eine Gleichverteilung der Bäume über die Fläche einschließt, kann diese Grundfläche als quadratisch angenommen werden.

Dabei wird davon ausgegangen, daß die horizontale Querschnittsfläche der Bäume in jeder Höhe kreisförmig ist. Diese Vereinfachung muß in einem eindimensionalen Modell getroffen werden, da man aufgrund der Voraussetzung der horizontalen Homogenität Abweichungen einzelner Individuen des Bestandes nicht berücksichtigen kann.

Die Bedeutung der für die mittlere Geometrie des Bestandes wichtigen Größen ist in Abb. 4.1 dargestellt. Für die Größen zur Beschreibung der mittleren Geometrie der Bäume des Bestandes wird auf Abb. 4.2 verwiesen. 
Der Druckabfall beim Durchströmen eines Bestandes (= Umströmen der Oberfläche aller Vegetationselemente) ist proportional der Entfernung $s$, die die Strömung im Bestand zurücklegt. Die infolge des Druckabfalles von der Strömung zu leistende Arbeit erhält man nach einem allgemeinen Zusammenhang durch Multiplikation des Druckabfalles $p_{v}$ mit dem Volumen $V_{o l}$ an Luft, in dem die Arbeit verrichtet wird. Diese Arbeit ist aber gleich der mittleren Widerstandskraft $F_{w}$ beim Durchströmen eines Baumes, multipliziert mit der zurückgelegten Weglänge $s$. Für den Druckabfall in einer Modellschicht k ergibt sich somit:

$$
p_{v, k}=\frac{F_{w, k} s}{V_{o l}} .
$$

Aufgrund der vorausgesetzten horizontalen Homogenität des Bestandes ist das durchströmte Volumen dem zurückgelegten Weg proportional und für eine Schicht k gilt nach dem Durch- und Umströmen von n Bäumen:

$$
\left(\frac{s}{V_{o l}}\right)_{k}=\frac{\Delta r n}{\Delta r^{2} h_{k} n} .
$$

Die Größe $h_{k}$ ist die Dicke der Modellschicht k. Damit bekommt man einen allgemeingültigen Ausdruck für $\left(\frac{s}{V_{o l}}\right)_{k}$.

So ergibt sich für den Druckabfall [46]:

$$
p_{v, k}=c_{d} \rho U_{k}^{2} \frac{A_{k}^{*}}{\Delta r h_{k}} .
$$

Die lokale zeitliche Geschwindigkeitsänderung in der atmosphärischen Schicht $k$ durch den aerodynamischen Widerstand läßt sich nach der folgenden Beziehung aus dem Druckabfall $p_{v, k}$ berechnen [46]:

$$
\frac{\partial U_{k}}{\partial t}=-\frac{1}{\rho} \frac{\partial p_{v, k}}{\partial r}
$$

Die horizontale Ableitung des Druckes $p_{v}$ läßt sich vereinfacht aus Gl. 4.7 berechnen nach:

$$
\frac{\partial p_{v, k}}{\partial r}=c_{d} \rho \frac{\partial A_{k}^{*}}{\partial r h_{k}} U_{k}^{2},
$$

(Ansatz nach Wilson \& Shaw, genannt bei [46]). Eigentlich müßten auch noch Terme mit den Ableitungen der Luftdichte, des aerodynamischen Widerstandskoeffizienten und der horizontalen Windgeschwindigkeit berücksichtigt werden. Diese müssen jedoch aufgrund des eindimensionalen Modells unter der Annahme horizontaler Homogenität weggelassen werden.

Ersetzt man den Differentialquotienten auf der rechten Seite durch einen Differenzenquotienten, so erhält man:

$$
\frac{\partial p_{v, k}}{\partial r} \approx c_{d} \rho \frac{\Delta A_{k}^{*}}{\Delta r} U_{k}^{2}
$$

und für die Änderung der horizontalen Windgeschwindigkeit $\frac{\partial U_{k}}{\partial t}$ bzw. $\frac{\Delta U_{k}}{\Delta t}$ :

$$
\frac{\partial U_{k}}{\partial t}=-c_{d} \frac{\Delta A_{k}^{*}}{\Delta r^{2} h_{k}} U_{k}\left|U_{k}\right| .
$$


Der Term $\frac{\Delta A_{k}^{*}}{\Delta r^{2}}$ ist die Projektion der Oberfläche aller Vegetationselemente einer Modellschicht auf die Horizontale, bezogen auf die einem Baum insgesamt zur Verfügung stehende Grundfläche. Bezieht man diese Größe nun noch auf die Höhe $h_{k}$ der Modellschicht, so erhält man ein Maß, wie "dicht" die Vegetationselemente angeordnet sind, die Vegetationsoberflächendichte, die wir mit $A_{\text {tree }, k}$ bezeichnen wollen. Die vertikale Verteilung der Vegetationsoberflächendichte wird im Modell berechnet nach [18]:

$$
A_{\text {tree }, k}= \begin{cases}0 & \text { für } z>h_{p} \\ \left(\Delta_{L A I, k}+s_{a, k}\right) & \text { für } z_{u}<z \leq h_{p} \\ \frac{d_{s}}{\Delta r^{2}} & \text { für } z \leq z_{u},\end{cases}
$$

wobei $d_{s}$ der Stammdurchmesser, $h_{p}$ die mittlere Bestandshöhe und $z_{u}$ die mittlere Höhe des Stammraumes ist. $\Delta_{L A I, k}$ bezeichnet die horizontale Projektion der einseitigen Blattfläche in der Schicht $k$, bezogen auf eine horizontale Projektionsfläche am Waldboden, hat also die Einheit $\mathrm{m}^{2} / \mathrm{m}^{2}$. Die Summe von $\Delta_{L A I, k}$ über alle Schichten des Kronenraumes wird als einseitiger Blattoder Nadelflächenindex LAI (leaf area index) bezeichnet. Sein Wert ist gleich der horizontalen Projektion aller Nadeloberflächen eines Baumes auf die horizontale Projektionsfläche der Baumkrone, dividiert durch diese Projektionsfläche. Eine Abschätzung von Ibrom (1993), genannt in [25] für den ganzseitigen Blattflächenindex ergab einen Wert von $20.4 \mathrm{~m}^{2} / \mathrm{m}^{2}$. Für das Verhältnis zwischen diesem ganzseitigen Blattflächenindex und LAI haben Riederer et al. (1988) (ebenfalls zitiert in [25]) einen Wert von 2.74 angegeben, so daß im Modell letztlich ein Wert von LAI $=7.45$ verwendet wird.

Die Einführung einer Größe $s_{a, k}$ zur Berücksichtigung des Anteiles aller anderen Vegetationselemente (Zweige, Äste usw.) an der horizontalen Projektion der Vegetationsoberfläche einer Bestandsschicht geht auf Deardorff 1978 zurück [8]. Es gibt in der Literatur jedoch unterschiedliche Ansichten, wie ein solcher Parameter zu berücksichtigen ist. In den im Rahmen dieser Arbeit durchgeführten Rechnungen ist $s_{a, k}$ der prozentuale Anteil der Äste und Zweige an der horizontalen Projektion der gesamten Vegetationsoberfläche einer Bestandsschicht. Es wird ein mittlerer Wert von $10 \%$ Anteil an der Nadeloberfläche des Baumes verwendet $\left(s_{a, k}=0.1-\right.$ siehe auch [18]). Nach Deardorff beschreibt $s_{a, k}$ den Zuwachs der horizontalen Projektion der Vegetationsoberfläche, wenn neben den Blättern auch der Anteil der Äste und Zweige berücksichtigt wird ( $s_{a, k}$ muß demnach größer als 1 sein - Deardorff verwendete $s_{a, k}$ =1.1). Atree, $k$ müßte sich also folgendermaßen berechnen:

$$
A_{\text {tree }, k}= \begin{cases}0 & \text { für } z>h_{p} \\ \Delta_{L A I, k} s_{a, k} & \text { für } z_{u}<z \leq h_{p} \\ \frac{d_{s}}{\Delta r^{2}} & \text { für } z \leq z_{u} .\end{cases}
$$

Bei eigenen Vergleichsrechnungen mit beiden Ansätzen konnten kaum Unterschiede in den Simulationsergebnissen festgestellt werden, so daß die Methode nach Inclán et al. bei allen weiteren Rechnungen angewendet wurde. Allerdings gibt es keine variierenden Angaben über die Größe $s_{a, k}$, was darauf 
hindeutet, daß eine meßtechnische Bestimmung dieser Größe für verschiedene Vegetationsbestände angebracht wäre. $w_{t r e e, k}$ ist der Durchmesser der von einem Baum in der Schicht k eingenommenen horizontalen Fläche. Die Größe $\Delta_{L A I, k}$ wird aus Gründen der numerischen Stabilität des Strahlungsmodells so gewählt, daß sie für jede Modellschicht im Bestand denselben Wert besitzt. Die Höhenabhängigkeit der Dichte der Vegetationsoberfläche wird dadurch berücksichtigt, daß die Abstände der Modellschichten im Kronenraum so gewählt werden, daß jede Schicht dieselbe Vegetationsoberfläche enthält. Ein solches Vorgehen gewährleistet die numerische Stabilität bei der Behandlung der kurz- und langwelligen Strahlung im Bestand (Ansatz nach Norman (1979), genannt in [18]). Nach der Vorgabe eines Wertes für die Vegetationso-

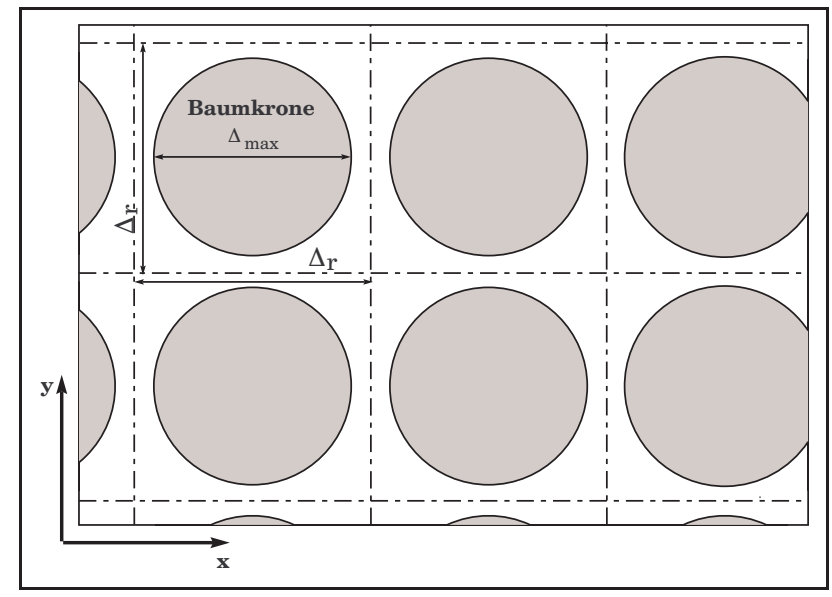

Abbildung 4.1: Schematische Darstellung der geometrischen Parameter des idealisierten Bestandes in der Aufsicht: $\Delta_{\max }=$ maxim. Kronendurchmesser

berfläche in jeder Schicht des Kronenraumes wird die Höhe der Modellschichten im Kronenraum iterativ durch Integration einer Beziehung für die einseitige Nadeloberfläche eines Baumes pro Volumeneinheit (einseitige Nadeloberflächendichte) bestimmt. Die vertikale Verteilung der Nadeloberflächendichte wurde 1986 bestimmt [12] und von Constantin 1993 durch eine RayleighVerteilung, einem Spezialfall der Poisson-Verteilung, angenähert [4]. Diese Beziehung hat die Form:

$$
\begin{gathered}
A n_{\text {tree }, k}=\frac{L A I}{\alpha_{n}} z_{r}^{2} \exp \left(-\alpha_{f} z_{r}\right), \\
\text { mit: } \mathrm{z}_{\mathrm{r}}=\frac{\mathrm{h}_{\mathrm{p}}-\mathrm{z}_{\mathrm{k}}}{\mathrm{h}_{\mathrm{p}}-\mathrm{z}_{\mathrm{u}}} .
\end{gathered}
$$

Dabei ist $\alpha_{n}$ eine Normierungsgröße mit $\alpha_{n}=5.7 \mathrm{~m}$ und $\alpha_{f}$ ein Formfaktor mit $\alpha_{f}=0.1695$. Der Wert $\alpha_{f}$ hängt dabei von den Werten für $h_{p}$ und $z_{u}$ ab, so daß das Integral von $A n_{\text {tree }}$ über die Vertikalkoordinate im Bereich von $h_{p}$ bis $z_{u}$ den einseitigen Blattflächenindex LAI ergibt. Die Vegetationsoberfläche in jeder Modellschicht beinhaltet neben der Nadeloberfläche auch noch alle anderen Vegetationselemente (Zweige, Äste, Stamm), was durch die Größe 


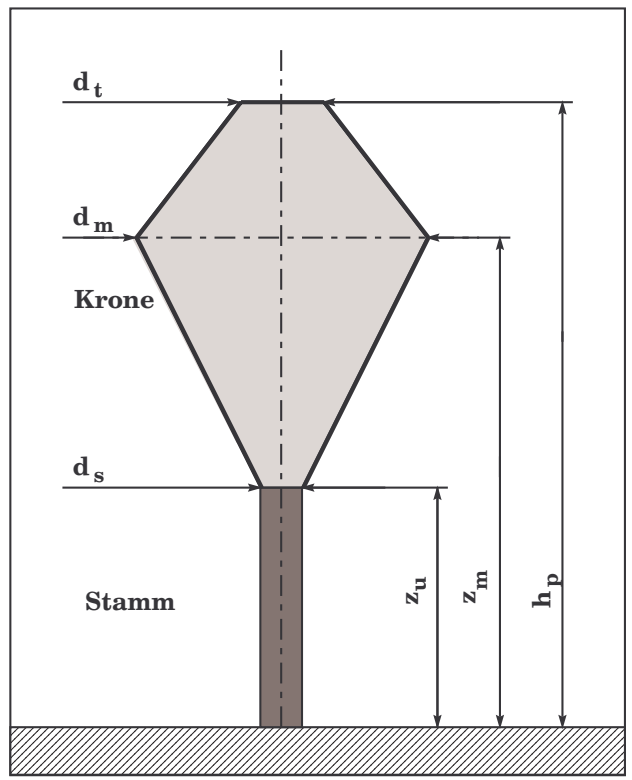

Abbildung 4.2: Schematische Darstellung der geometrischen Parameter eines Modellbaumes in der Seitenansicht. Es bedeuten: $d_{t}$ - Kronendurchmesser in Höhe des Kronendaches, $d_{m}$ - maximaler Kronendurchmesser, $d_{s}$ - Stammdurchmesser, $h_{p}$ - Höhe des Bestandes, $z_{m}$ - Höhe, in der der maximale Kronendurchmesser erreicht wird, $z_{u}$ - Höhe des Stammraumes

$s_{a}\left[\mathrm{~m}^{2} / \mathrm{m}^{2}\right]$ berücksichtigt wird. Die Anwendung von Gleichung 4.11 zur Berechnung der Windgeschwindigkeit im Bestand kann jedoch zu numerischen Problemen führen, wenn man nicht die einzelnen Komponenten der Windgeschwindigkeit, sondern nur den Betrag betrachtet, da dann unter Umständen ein negativer Betrag der Windgeschwindigkeit auftreten kann. Das kann z.B. notwendig werden, wenn man keine Angaben über die geostrophische Windgeschwindigkeit hat und so die Windrichtungsänderungen infolge des Zusammenspiels von Coriolis- und Reibungs- bzw. Widerstandskraft nicht berechnen kann.

Bemerkung: Wenn nur der Betrag der Windgeschwindigkeit betrachtet wird, können die zweiten Terme auf der rechten Seite der Gleichungen 4.1 und 4.2 weggelassen werden, da die Wirkung der Corioliskraft nur in einer Windrichtungsänderung und nicht in einer Änderung des Betrages der Windgeschwindigkeit zum Ausdruck kommt (,,Scheinkraft"), wie man durch Berechnung der kinetischen Energie vor und nach dem Zeitschritt $\Delta t$ nachprüfen kann.

Um das beschriebene Problem zu umgehen, wurde von Stull et al. (1993) eine andere Vorgehensweise vorgeschlagen, bei der die obige Gleichung in ihrer integrierten Form verwendet wird [43] und man erhält für die Komponente 


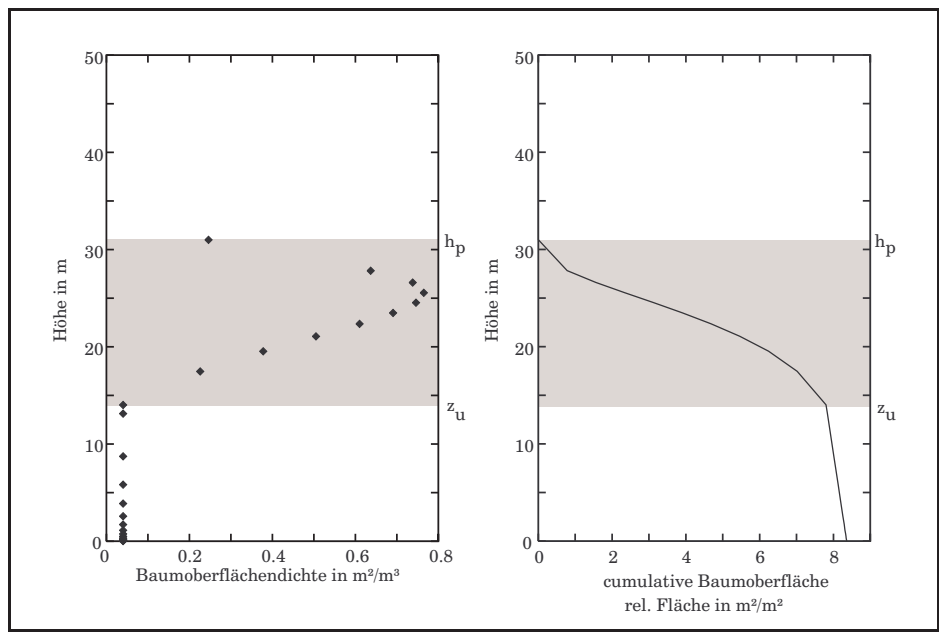

Abbildung 4.3: Vertikale Verteilung der Baumoberflächendichte und der cumulativen Baumoberfläche für den F1-Bestand im Solling - die grau unterlegten Bereiche kennzeichnen den Kronenraum

der Windgeschwindigkeit in Richtung der x-Koordinate $\Delta u_{k}$ :

$$
\Delta u_{k}=-\frac{0.5 c_{d} A_{t r e e, k} u_{k}(t) U_{k}(t) \Delta t}{1+0.5 c_{d} A_{t r e e, k} U_{k}(t) \Delta t} .
$$

Eine Herleitung findet sich bei Inclán et al. [18]. Diese Beziehung gilt ana$\log$ auch für die Komponente der Windgeschwindigkeit in Richtung der yKoordinate $\Delta v_{k}$. Sie löst das beschriebene Problem: Die Änderung des Betrages der horizontalen Windgeschwindigkeit $\Delta U_{k}$ ergibt sich unter Anwendung des Satzes des Pythagoras aus den Änderungen der Komponenten in $\mathrm{x}$ - und y-Richtung. Damit erhält man für $\Delta U_{k}$, die der Gl. 4.15 ähnelt, nur daß auf der rechten Seite $u_{k}$ durch $U_{k}$ ersetzt werden muß. So ergibt sich also $\left|\Delta U_{k}\right|$ durch Multiplikation des Betrages der horizontalen Windgeschwindigkeit $U_{k}$ mit einem Faktor, der immer kleiner oder gleich 1 ist, so daß schließlich $U_{k}$ nicht mehr negativ werden kann.

\subsection{Nachlaufturbulenz}

Infolge der Turbulenz im Nachlauf der Vegetationselemente kommt es zu einer Änderung $\Delta E$ der turbulenten kinetischen Energie E. Diese Änderung ist proportional zum Formwiderstand. Eine Parametrisierung von $\Delta E$ basiert auf der Überlegung, daß die gesamte kinetische Energie, die der mittleren Bewegung durch den aerodynamischen Widerstand entzogen wurde, in Energie der turbulenten Bewegung übergeht und erst dann der Dissipation in Wärmeenergie unterliegt. Diese zusätzliche turbulente kinetische Energie trägt zu den turbulenten vertikalen Eigenschaftsflüssen bei. Damit muß die turbulente kinetische Energie infolge des Nachlaufes hinter den Vegetationselementen auch dem Quadrat der Geschwindigkeitsänderungen durch den aerodynamischen 
Widerstand proportional sein und man erhält die folgende allgemeine Beziehung:

$$
\Delta Y \sim \Delta E \sim\left(\left.\Delta u\right|_{\text {FormDrag }}\right)^{2} \sim\left(c_{d} A_{\text {tree }} U^{2} \Delta t\right)^{2} .
$$

Als einfacher Ansatz zur mathematischen Beschreibung kann eine nichtlokale Approximation für die Änderung $\Delta Y_{i j}$ der Elemente des Mischungspotentials nach Inclán et al. benutzt werden [18]:

$$
\Delta Y_{i j}=0.5 c_{d}\left[A_{\text {tree }, i} U_{i}^{3}+A_{\text {tree }, j} U_{j}^{3}\right]\left(\frac{l}{\Delta z_{i j}}\right) \Delta t \quad \text { für i } \neq \mathrm{j},
$$

mit:

1 - Längenscale der Pflanzenelemente [30]. Diese Parametrisierung geht auf Messungen und numerische Vergleichsrechnungen von Raupach und Shaw (1982) an einem Modellwaldbestand zurück. Die Größe l beschreibt den Durchmesser aller Vegetationselemente, die entsprechend ihrer Oberfläche berücksichtigt werden. Für $l$ wird in den Rechnungen ein Wert von $l=0.005 \mathrm{~m}$ verwendet [18]. Ist $\Delta z_{i j}$ groß gegenüber l, d.h. $Y_{i j}$ beschreibt den Austausch zwischen zwei weit auseinander liegenden Schichten, von denen eine innerhalb des Bestandes liegt, so leistet die Nachlaufturbulenz nur einen geringen Beitrag zum Mischungspotential. Der Anteil des Mischungspotentials infolge der Nachlaufturbulenz ist umso größer, je kleiner der Abstand zwischen den Schichten wird, ein durchaus realistisches Verhalten, da damit die schnellere Dissipation der Nachlaufwirbel aufgrund ihrer geringen Größe berücksichtigt wird. Die Wirkung elastischer Verformungen der Vegetationselemente auf den turbulenten Austausch in der Luft braucht dabei nicht gesondert betrachtet zu werden, da sie bei der meßtechnischen Bestimmung des aerodynamischen Widerstandes miterfaßt werden und damit im aerodynamischen Widerstandskoeffizienten implizit berücksichtigt wird. Trotzdem ist die vorliegende Parametrisierung nur ein erster einfacher Ansatz [18].

\subsection{Der Einfluß auf vertikale turbulente Umlagerun- gen}

Die vertikalen turbulenten Austauschprozesse werden von den Pflanzenelementen beeinflußt. Die damit verbundenen Effekte lassen sich im Falle der nichtlokalen transilienten Turbulenzschließung leichter berücksichtigen, als z. B. in der K-Theorie, bei der Annahmen über die Veränderungen der Mischungsweglängen gemacht werden müssen. Zwischen zwei beliebigen Modellschichten beschreibt die TTT die Wirkung von Wirbeln, deren Durchmesser etwa dem Abstand zwischen diesen beiden Schichten entspricht. Befinden sich beide oder auch nur eine Schicht unterhalb der Obergrenze der Vegetation, so ist diese Wirkung durch das Vorhandensein der Vegetationselemente beeinträchtigt. Dort, wo sich keine Vegetationselemente befinden (z. B. zwischen den Bäumen), wird der vertikale turbulente Austausch nicht beeinträchtigt, an anderen Stellen (z. B. Stämme) erfolgt überhaupt kein vertikaler turbulenter Austausch, an wieder anderen Stellen (z. B. an Blättern, Ästen und Zweigen) wird dieser Austausch ähnlich der horizontalen Strömung durch den aerodynamischen Widerstand behindert. Die Verringerung der vertikalen Transporte 
in jeder Modellschicht ist also negativ korreliert mit dem Verhältnis der Projektion der Fläche der gesamten Vegetationsoberfläche eines Baumes in dieser Schicht auf die Horizontale zur horizontalen Gesamtfläche, die diesem Baum insgesamt zur Verfügung steht. Die benötigten Werte für die Vegetationsoberfläche pro Schicht und die Gesamtfläche können aus Feldmessungen und aus Messungen der Baumdichte (Bäume pro ha) gewonnen werden (Lee \& Black, 1993; Cohen et al. 1985 u. a., genannt in [18]). In Anlehnung an die Wechselwirkung zwischen vertikalem Transport und Vegetationselementen ( „Interferenz" ) werden die genannten Verhältnisse nach Stull 1990 auch Interferenzkoeffizienten genannt. Sind alle benötigten Werte über die mittlere Geometrie der Bäume bekannt, so lassen sich die Interferenzkoeffizienten $\Gamma_{k}$ für jede Schicht $k$ berechnen nach [41],[18]:

$$
\Gamma_{k}= \begin{cases}0 & \text { für } z>h_{p} \\ \frac{\pi\left(0.5 w_{\text {tree }, k}\right)^{2}}{\Delta r_{2}}\left(\Delta_{L A I}+s_{a}\right) & \text { für } z_{u}<z<h_{p} \\ \frac{\pi\left(0.5 D_{t}\right)^{2}}{\Delta r_{2}} & \text { für } z \leq z_{u} .\end{cases}
$$

Die Werte von $\Gamma$ variieren zwischen 0 (keine Behinderung des vertikalen turbulenten Transportes) und 1 (kein vertikaler turbulenter Transport in der Schicht k). Der Austausch zwischen der Schicht k und der Schicht k + 1 wird dann um $100 \times \Gamma_{k} \%$ verringert, $d$. h. der entsprechende Koeffizient des massengewichteten Mischungspotentials muß mit dem Faktor $\left(1-\Gamma_{k}\right)$ multipliziert werden. Wird der Austausch zwischen der Schicht k und der Schicht $k+2$ betrachtet, so wird dieser nochmals, und zwar um $100 \times \Gamma_{k+1} \%$ verringert, der entsprechende Koeffizient des massengewichteten Mischungspotentials muß nun mit dem Faktor $\left(1-\Gamma_{k}\right)\left(1-\Gamma_{k+1}\right)$ multipliziert werden. Die Gesamtinterferenz für einen Wirbel, der sich von einer Quellschicht j zu einer Zielschicht i bewegt, ergibt sich also durch das Produkt aller Werte $\left(1-\Gamma_{k}\right), k=j, i-1$ über die gesamte vertikale Distanz zwischen beiden Schichten [39].

Für die Eddies, welche Luft von höheren zu niederen Schichten transportieren, müssen die Elemente des massengewichteten Mischungspotentials $Y_{\omega, i j}$ ersetzt werden durch:

$$
Y_{\omega, i j} \rightarrow Y_{\omega, i j} \prod_{k=i}^{j-1}\left[1-\Gamma_{k}\right] \text { für } \mathrm{j}>\mathrm{i} .
$$

Im umgekehrten Falle gilt:

$$
Y_{\omega, i j} \rightarrow Y_{\omega, i j} \prod_{k=j}^{i-1}\left[1-\Gamma_{k}\right] \quad \text { für } \mathrm{j}<\mathrm{i} .
$$




\section{Kapitel 5}

\section{Das Modell}

\subsection{Aufbau des Atmosphären - und Bestandsmodells}

Das eindimensionale vertikal aufgelöste Modell der atmosphärischen Grenzschicht und des Bestandes besteht aus einer für die Dauer einer Rechnung konstanten, sonst jedoch in gewissen Grenzen wählbaren Anzahl von Schichten. Die Höhe der Modellschichten ist zeitlich konstant, nimmt jedoch im allgemeinen von der Grenze zum Bodenmodell bis zur Obergrenze des Atmosphärenmodells zu.

Eine Ausnahme bildet in dieser Hinsicht der Kronenraum - die Schichthöhen sind hier so gewählt, daß jede Modellschicht dieselbe Nadeloberfläche enthält. Eine solche Einteilung wird notwendig, um die numerische Stabilität bei der Behandlung der Terme für die Berücksichtigung der kurz- und langwelligen Strahlung im Kronenraum zu verbessern (diese Vorgehensweise geht zurück auf Norman (1979), genannt in [18]). Dadurch wird gewährleistet, daß jede Modellschicht innerhalb des Kronenraumes dieselbe Menge an kurzwelliger Sonnenstrahlung absorbiert (für den kurzwelligen Extinktionskoeffizienten wird ein im Kronenraum höhenkonstanter Wert verwendet). Auf diese Weise bleibt das Modell während der Rechnung energetisch stabil.

Die Dicke der Modellschichten nimmt oberhalb 3000 m Modellhöhe nicht mehr $\mathrm{zu}$, und wird mit $500 \mathrm{~m}$ bis zur Modellobergrenze von $4000 \mathrm{~m}$ konstant gehalten, um eine ausreichende Genauigkeit der Rechnung zu gewährleisten.

Die Höhe der Modellobergrenze wurde auf $4000 \mathrm{~m}$ festgelegt, während die Modellergebnisse nur bis zu einer Höhe von 3000 m ausgewertet werden, um das Problem des oberen Modellrandes im Rahmen der transilienten Turbulenzschließung (die turbulenten Transporte durch die Obergrenze des Simulationsgebietes verschwinden aufgrund der Definition des transilienten Schließungsansatzes) zu reduzieren. 


\subsection{Modellparameter und Randwerte}

Das benutzte Grenzschichtmodell wurde für die Simulation der Grenzschicht über und in einem Waldbestand im Solling (Weserbergland - $80 \mathrm{~km}$ südwestlich von Hannover - 51 $46^{\prime}$ N, $9^{\circ} 35^{\prime}$ E, 505 m über NN (Höhenangabe nach [12])) angepaßt. Das Mittelgebirge Solling bildet eine nach allen Seiten flach ab-

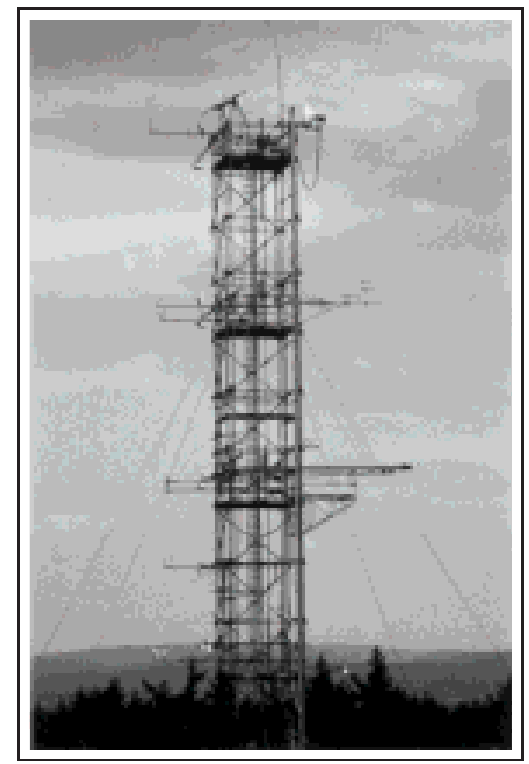

Abbildung 5.1: 50 m-Meßturm des Institutes für Bioklimatologie im Fichtenbestand (F1-Fläche) im Solling

fallende Erhebung von etwa $500 \mathrm{~km}^{2}$ Größe und erreicht eine maximale Höhe von 528 m über NN [4]. Das Klima des Sollings kann als subatlantisches Mittelgebirgsklima bezeichnet werden. Die mittlere Jahresniederschlagsmenge liegt bei $1080 \mathrm{~mm}$ mit einem Maximum im Winter (Dezember/Januar) und einem zweiten Maximum im Sommer (Juli/August). Die klimatologische Jahresmitteltemperatur beträgt $6.4^{\circ} \mathrm{C}$. Fröste können noch im April und Mai auftreten; die höchsten Temperaturen $\left(27-28{ }^{\circ} \mathrm{C}\right)$ werden im Juli gemessen (alle Klimawerte nach Szarenko 1990, genannt bei [4]).

Der betrachtete Bestand besteht aus im Jahre 1998114 Jahre alten Fichten (Picea abies) mit einer Bestandsdichte von 461 Bäumen pro ha [12]. Die Bestandshöhe beträgt etwa $31 \mathrm{~m}$ (Wert gilt für 1998 - da das Baumwachstum anhält). Unter dem Bestand existiert eine spärliche Strauchschicht über einer gut ausgebildeten Krautschicht („,Siebenstern - Fichtenforst") [12].

Das Untersuchungsgebiet ist nicht ideal homogen - in etwa 200 m Entfernung zum Meßturm schließt sich in südlicher und südwestlicher Richtung ein rund $30 \mathrm{~m}$ hoher Buchenbestand und in etwa $200 \mathrm{~m}$ Entfernung in nordwestlicher Richtung ein junger $10 \mathrm{~m}$ hoher Fichtenbestand an.

Im betrachteten Bestand werden am $50 \mathrm{~m}$ Meßturm des Institutes für Bioklimatologie der Universität Göttingen (Abb. 5.1) und am Boden regelmäßi- 
ge Messungen verschiedener meteorologischer Größen (Niederschlag, Temperatur, Windgeschwindigkeit, spezifisch Feuchte, Strahlung) durchgeführt. Die Werte dieser Größen werden für die Modellinitialisierung bei instationärer Rechnung bzw. für die Vorgabe der Profile bei stationärer Rechnung verwendet. Für die Messung der Windgeschwindigkeit in möglichst großer Höhe über

\begin{tabular}{|c||c|c|c|c|c|}
\hline Höhe $(\mathrm{m})$ & $v_{h}(\mathrm{~m} / \mathrm{s})$ & $\alpha\left(^{\circ}\right)$ & $t\left({ }^{\circ} \mathrm{C}\right)$ & $r_{f}(\%)$ & $p(\mathrm{hPa})$ \\
\hline \hline 1 & $\mathrm{x}$ & 0 & $\mathrm{x}$ & 0 & 0 \\
\hline 2 & 0 & 0 & 0 & 0 & $\mathrm{x}$ \\
\hline 3 & $\mathrm{x}$ & 0 & $\mathrm{x}$ & $\mathrm{x}$ & 0 \\
\hline 5 & $\mathrm{x}$ & 0 & $\mathrm{x}$ & 0 & 0 \\
\hline 9 & $\mathrm{x}$ & 0 & $\mathrm{x}$ & $\mathrm{x}$ & 0 \\
\hline 15 & $\mathrm{x}$ & 0 & $\mathrm{x}$ & 0 & 0 \\
\hline 21 & $\mathrm{x}$ & 0 & $\mathrm{x}$ & $\mathrm{X}$ & 0 \\
\hline 27 & $\mathrm{x}$ & 0 & $\mathrm{x}$ & 0 & 0 \\
\hline 33 & $\mathrm{x}$ & 0 & $\mathrm{x}$ & 0 & 0 \\
\hline 35 & 0 & $\mathrm{x}$ & 0 & 0 & 0 \\
\hline 39 & $\mathrm{x}$ & 0 & $\mathrm{x}$ & 0 & 0 \\
\hline 45 & $\mathrm{x}$ & 0 & $\mathrm{x}$ & 0 & 0 \\
\hline 51 & $\mathrm{x}$ & 0 & $\mathrm{x}$ & 0 & 0 \\
\hline 52 & 0 & $\mathrm{x}$ & 0 & 0 & 0 \\
\hline 56 & $\mathrm{x}$ & 0 & 0 & 0 & 0 \\
\hline
\end{tabular}

Tabelle 5.1: Überblick über die als Eingabeparameter des transilienten Modells verwendeten am $50 \mathrm{~m}$ - Meßturm gewonnenen Werte physikalischer Größen. Es bedeuten: x - Messungen werden vorgenommen, 0 - keine Messung in dieser Höhe; $v_{h}$ - horizontale Windgeschwindigkeit, $\alpha$ - Windrichtung, $t$ - Trockentemperatur der Luft, $r_{f}$ - relative Feuchte der Haarhygrometer, $p$ - Luftdruck. Hinzu kommen noch die Niederschlagsmessungen über dem Bestand und Strahlungsmessungen über- und unterhalb des Kronenraumes.

dem Bestand ist ein Schalenkreuzanemometer an einer Mastverlängerung in einer Höhe von $56 \mathrm{~m}$ angebracht. Die übrigen Anemometer sind an $2.5 \mathrm{~m}$ langen, in Richtung Norden zeigenden Auslegern an der Nordwestecke des Turmes befestigt. So wird die Gefahr einer Registrierung des vom Turm beeinflußten Windfeldes bei den vorherrschenden westlichen Windrichtungen minimiert [4], [5].

Die Tabelle 5.1 gibt einen Überblick über die für das Betreiben des Modells verwendeten Meßwerte. Bei den Trockentemperaturen der Luft handelt es sich um die am trockenen Einlaß der Aspirationspsychrometer gemessenen Temperaturen der Luft. Die am feuchten Einlaß der Psychrometer gemessenen Feuchttemperaturen der Luft werden nicht für die Bestimmung der spezifischen Feuchte $q$ der Luft verwendet, da ein Trockenlaufen der Psychrometer nicht immer verhindert werden kann. Stattdessen wird $q$ bestimmt aus der relativen Feuchte des Haarhygrometers und der spezifischen Sättigungsfeuchte $q_{s}$ der Luft in der selben Höhe. Da $q_{s}$ auch vom aktuellen Luftdruck $p$ abhängt, muß dieser aus dem gemessenen Luftdruck in $2 \mathrm{~m}$ Höhe abgeleitet werden. Diese Interpolation geschieht mittels der barometrischen Höhenformel unter Berücksichtigung der Temperaturabhängigkeit der Luftdruckabnahme mit der Höhe. Die geringfügige Abhängigkeit der Druckabnahme mit der Höhe von der spezifischen Feuchte $q$ wird dabei unter Zugrundelegung eines angenommenen höhenkonstanten Wertes für $q$ von $5 \mathrm{~g} / \mathrm{kg}$ berücksichtigt, da das wahre Pro- 
fil von $q$ ja erst nach der Berechnung des Luftdruckprofiles bestimmt werden kann.

Über dem Bestand werden in 39 m Höhe die von der Atmosphäre nach unten gerichtete langwellige Strahlung (atmosphärische Gegenstrahlung - Wellenlängen ab $3500 \mathrm{~nm}$ ) und die Summe von kurzwelliger direkter und diffuser Strahlung (Globalstrahlung - Wellenlängenbereich von 360 bis $760 \mathrm{~nm}$ ), sowie die Summe der direkten und diffusen photosynthetisch aktiven Strahlung (PAR - Wellenlängenbereich von 400 bis $700 \mathrm{~nm}$ ) gemessen [25]. Die PAR wird zur Bestimmung der Stomatawiderstände im Kronenraum benötigt. Da die Sensoren zur Messung der PAR-Strahlung nicht wie die anderen Sensoren regelmäßig geeicht werden, werden die Werte der PAR aus den gemessenen Werte der Globalstrahlung abgeschätzt. Energiemäßig macht die PAR einen Anteil von etwa $45 \%$ der Globalstrahlung aus ${ }^{1}$.

Unter dem Kronenraum stehen in 2 m Höhe Meßwerte der einfallenden kurzwelligen Strahlung und der Gesamtstrahlungsbilanz zur Verfügung. Diese Meßwerte stellen horizontale Mittelwerte dar, die mittels auf einer $10 \mathrm{~m}$ langen Strahlungsschiene montierten Geräten gemessen werden, die sich ständig längs dieser Schiene hin- und herbewegen [25]. Diese Meßwerte werden zu Vergleichszwecken zur Überprüfung des Strahlungsteiles des Modells verwendet.

$\mathrm{Zu}$ diesen Meßwerten atmosphärischer Größen kommen noch die Werte der gemessenen Bodentemperaturen, die zur Vorgabe des Bodentemperaturprofils verwendet werden. Die Bodentemperatur wird mit Hilfe von Widerstandsthermometern in 5 Bodentiefen von 2, 5, 10, 50 und $100 \mathrm{~cm}$ gemessen. Aufgrund fehlender Messungen zwischen 2 und $3 \mathrm{~m}$ Tiefe wird die Bodentemperatur in diesem Bereich als tiefenunabhängig vorgegeben, wobei sich die Temperaturwerte an den Meßwerten in $2 \mathrm{~m}$ Tiefe orientieren. Aus demselben Grunde wird für die Bodenfeuchte ein realitätsnahes theoretisches Profil angenommen (die Bodenfeuchte wird im Profil nicht regelmäßig wie die anderen Größen gemessen).

Für die Initialisierung des Modells und für die Festlegung der oberen Randwerte werden Analyse- und Prognosedaten des Deutschlandmodells des DWD für den Gitterpunkt Solling (Modellkoordinaten: 51 $42^{\circ}$ N, $9^{\circ} 30^{\prime}$ E, Höhe $=368$ $m$ ü. NN) verwendet. Die dazu notwendigen Daten wurden vom Deutschen Wetterdienst freundlicherweise kostenlos zur Verfügung gestellt. Im interessierenden Höhenbereich $(0-4000 \mathrm{~m})$ standen die Daten im Abstand von 3h für 20 Schichten als Schichtmittelwerte zur Verfügung. Durch die Verwendung einer Sigma - Vertikalkoordinate ${ }^{2}$ im Wetterdienstmodell lassen sich keine festen Höhen für die Schichten angeben, auf denen diese Daten vorliegen. Die folgenden Größen wurden für das transiliente Modell als Eingabeparameter verwendet: Luftdruck [hPa], Lufttemperatur $\left[{ }^{\circ} \mathrm{C}\right]$, spezifische Feuchte $[\mathrm{g} / \mathrm{kg}$, horizontale Windgeschwindigkeit [Knoten] und Windrichtung [ $\left.{ }^{\circ}\right]$.

Da sich die geographischen Koordinaten des Modellgitterpunktes nicht wesentlich von denen der Meßfläche im Solling unterscheiden, erscheint eine Kombination von gemessenen Daten in und knapp über dem Bestand mit den Analyse - und Prognosedaten des Deutschlandmodells sinnvoll. Die unterschiedli-

\footnotetext{
${ }^{1}$ Oltchev 1998, persönliche Mitteilung

${ }^{2}$ Sigma $(\sigma)$ ist der relative Luftdruck = Luftdruck in der jeweiligen Höhe bezogen auf den Bodenluftdruck.
} 
chen Höhen über NN des Modellgitterpunktes und der realen Meßfläche lassen sich dabei auf die starke Glättung der Modellorographie gegenüber der Realität zurückführen. Dies ist eine Folge der Gitterweite des Modellgitters in der horizontalen Ebene $(10 \mathrm{~km} \times 10 \mathrm{~km})$.

$\mathrm{Zu}$ Beginn der Rechnung müssen die Profile der Lufttemperatur, des Luftdruckes, der horizontalen Windgeschwindigkeit und der spezifischen Luftfeuchte, sowie das Profil der Bodentemperatur vorgegeben werden. Die Anfangsprofile werden über dem Bestand aus den für 0:00 Uhr jeden Tag vorliegenden Analysewerten des Deutschlandmodells des DWD für den Gitterpunkt Solling linear auf die Modellschichten extrapoliert und im Bestand aus den Viertelstunden-Mittelwerten ebenfalls linear auf die Modellschichten extrapoliert. Zwischen den Meßwerten und den Analysewerten des Deutschlandmodells besteht eine Lücke. Diese wird in Ermangelung eines Anpassungsmodells ebenfalls durch lineare Interpolation geschlossen.

Im Verlauf einer Simulation werden nur noch die oberen Randwerte (4000 m Höhe) der Profile der oben genannten physikalischen Eigenschaften der Atmosphäre angepaßt. Dazu werden die alle 3 Stunden vorliegenden Vorhersagedaten des Deutschlandmodells linear auf die Modellzeit des transilienten Modells extrapoliert.

Der Ablauf der Grenzschichtmodellierung ist in Abb. 5.2 nochmals zusammenfassend dargestellt. Das Zeitlimit ist erreicht, wenn die vorzugebende Dauer der Simulation überschritten wird. Die für die Modellsimulationen verwendeten Parameter sind in Tabelle 5.2 dargestellt. Die pflanzenphysiologischen Parameter wurden aus Messungen bestimmt. Ein atmosphärisches Grenzschicht-

\begin{tabular}{|c|c|c|c|}
\hline Bezeichnung & Beschreibung & Wert & Quelle \\
\hline LAI & integr. Blattfl.-Index (einseitig) & 7.45 & [25] \\
\hline$t_{d}$ & Baumdichte & 461 Bäume/ha & [12] \\
\hline$\Delta r$ & $\begin{array}{l}\text { mittl. Seitenlänge der } \\
\text { horiz. Bodenfläche/Baum }\end{array}$ & $4.65 \mathrm{~m}$ & " \\
\hline$\Delta_{L A I}$ & Blattflächenindexinkrement & 0.745 & modellspezifisch! \\
\hline$z_{u}$ & Stammraumhöhe & $14 \mathrm{~m}$ & {$[12]$} \\
\hline$h_{p}$ & Bestandshöhe & $31 \mathrm{~m}$ & " \\
\hline$z_{m}$ & Kronendurchmesser in: & $27.5 \mathrm{~m}$ & [18] \\
\hline$d_{s}$ & Stammdurchmesser & $0.40 \mathrm{~m}$ & [12] \\
\hline$d_{m}$ & max. horiz. Kronendurchmesser & $4.00 \mathrm{~m}$ & " \\
\hline$d_{t}$ & horiz. Kronendurchmesser in Höhe $h_{p}$ & $2.4 \mathrm{~m}$ & [18] \\
\hline$l_{b}$ & mittlerer Nadeldurchmesser & $0.005 \mathrm{~m}$ & " \\
\hline$s_{a}$ & $\begin{array}{l}\text { Anteil von Ästen u. Zweigen } \\
\text { an der Gesamtbaumoberfläche }\end{array}$ & 0.1 & " \\
\hline$c_{d}$ & aerodynam. Widerstandskoeff. & 0.2 & " \\
\hline$m_{b}$ & $\begin{array}{l}\text { Verhältnis der Gesamtnadelfläche } \\
\text { zu deren horizontaler Projektion }\end{array}$ & 2.74 & {$[25]$} \\
\hline
\end{tabular}

Tabelle 5.2: Im Modell verwendete pflanzenphysiologische Parameter im Bereich des Stamm- und Kronenraumes.

modell kann prinzipiell in 2 verschiedenen Moden betrieben werden. Der eine Modus besteht in einer Berechnung der Profile der gewünschten Größen zu einem festgelegten Zeitpunkt (stationäre Rechnung), der andere Modus in einer Berechnung der Profile zusammen mit ihrer zeitlichen Veränderlichkeit (instationäre Rechnung). Bei einem stationären Modellauf besteht nun bei dem 


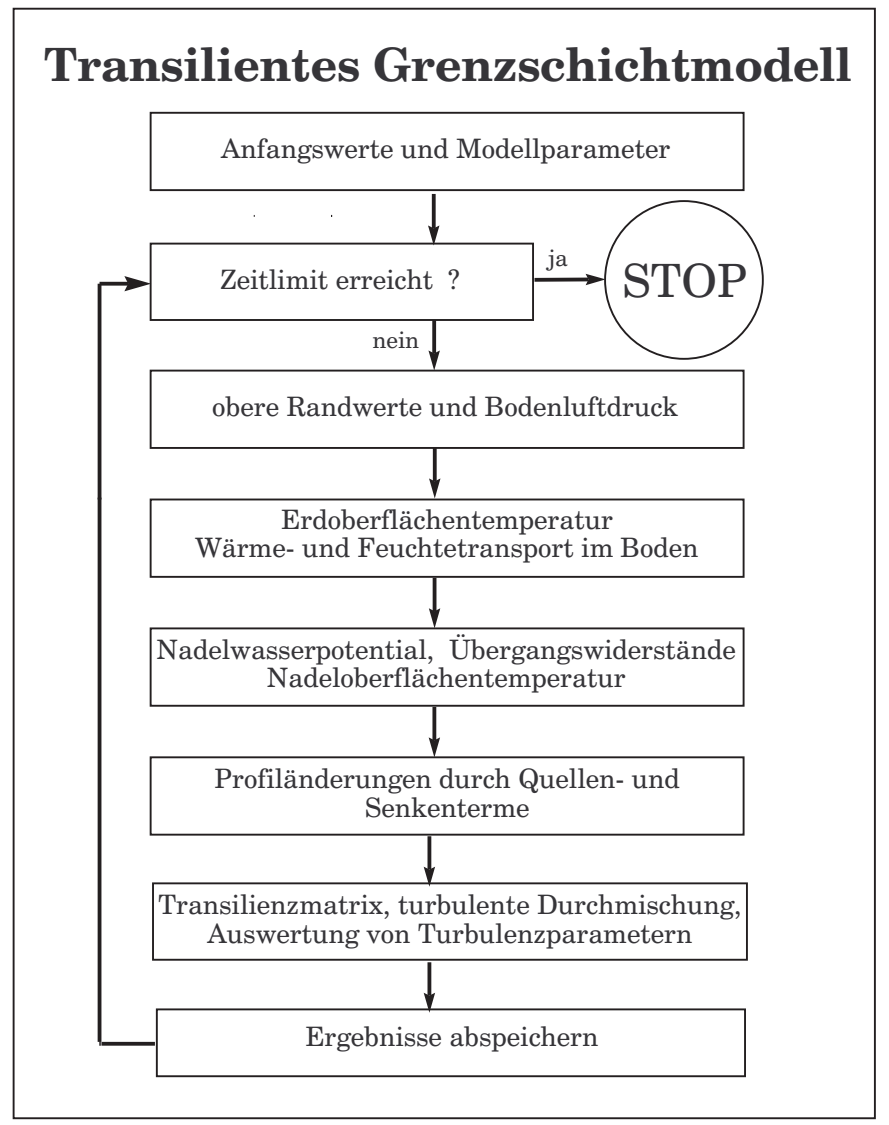

Abbildung 5.2: Schema des Grenzschichtmodells mit transilienter Turbulenzschließung

Modell mit transilienter Turbulenzschließung das Problem der Abfolge von Destabilisierung, Berechnung der Transilienzmatrix und Berechnung der neuen Profile (Stabilisierung) - ein Modellierungsschema, welches für instationäre Rechnungen zugeschnitten ist. Deshalb wird für die stationären Rechnungen eine Iteration der folgenden Art und Weise durchgeführt. Nach dem Festlegen der Anfangsprofile rechnet das Modell bei unveränderten oberen und unteren Randwerten bis zum Erreichen eines stationären Zustandes (keine Zeitintegration!). Die Bezeichnung untere Randwerte schließt hier alle Turmmeßwerte sowie die anderen gemessenen Größen ein. Auch die Anfangswerte der Profile aus der DWD - Modellanalyse oberhalb 2000 m Höhe über Grund werden dabei konstant gehalten, um den Charakter des atmosphärischen Zustandes oberhalb der Grenzschicht zu erhalten. Um ein Maß für die Feststellung eines stationären Zustandes der Simulation zu erhalten in das der Einfluß möglichst vieler Größen eingeht, wurde die Mischungsweglänge (berechnet nach Gl. 3.56) bzw. das Integral der Mischungsweglänge im gesamten Modellbereich herangezogen. Das heißt, der stationäre Zustand ist erreicht, wenn die Summe der Änderungen der Mischungswege in jeder Schicht von einem Modellschritt 


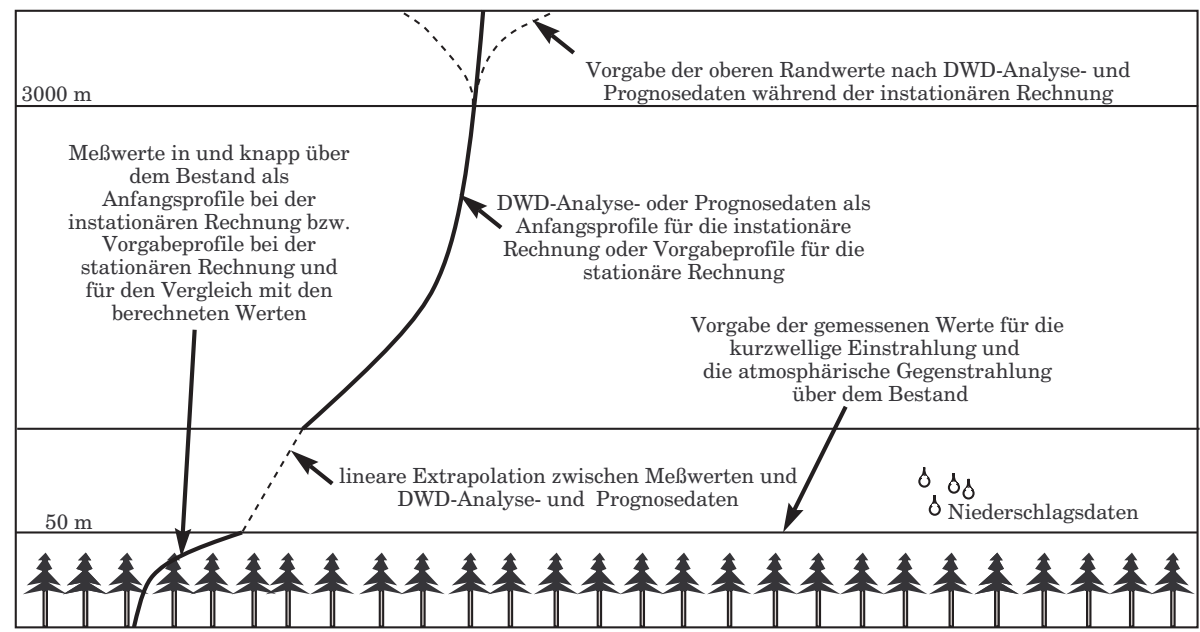

Abbildung 5.3: Schema der Verwendung der Meßwerte und der Analyse - und Prognosedaten des Wetterdienstmodells im atmosphärischen Teil des transilienten Grenzschichtmodells mit Waldbestand (Darstellung nicht maßstabsgerecht)

zum nächsten nur noch wenig ändert. In unserem Fall wurde „wenig“ mit maximal $1 \%$ der Summe der Mischungsweglängen in jeder Schicht definiert. Diese Vorgehensweise hat auch den Vorteil, daß eventuelle Sprünge beim Übergang der Vorgabeprofile von den Meßwerten zu den Analyseprofilen bzw. Prognoseprofilen des Wetterdienstmodells ausgeglichen werden.

Bei den instationären Modelläufen wird zunächst auf der Grundlage der Anfangsprofile eine stationäre Rechnung ausgeführt, bevor mit der eigentlichen Zeitintegration begonnen wird.

Die Abb. 5.3 faßt den Einsatz der vorhandenen Daten im Modell schematisch zusammen.

In den folgenden Abschnitten soll auf die im Modell berücksichtigten physikalischen Prozesse näher eingegangen werden. Die Abb. 5.4 gibt dazu einen Überblick über die wichtigsten im Modell berücksichtigten Prozesse.

\subsection{Bestimmung der Erdoberflächentemperatur}

Das Bodenmodell setzt sich aus 2 Teilen zusammen - dem Bodentemperaturund dem Bodenfeuchtemodell, welche beide dasselbe Modellgitter benutzen. Es erstreckt sich bis in $3 \mathrm{~m}$ Tiefe. Entsprechend der mit zunehmender Bodentiefe abnehmenden zeitlichen Variabilität der Bodentemperatur und -feuchte nimmt die Dicke der Bodenschichten mit der Tiefe exponentiell zu.

Die Energiebilanzgleichung an der Erdoberfläche wird im Modell in der Form:

$$
F_{s, 0}+F_{l, 0}^{\downarrow}-F_{l, 0}^{\uparrow}=P_{0}+L E_{0}+B_{0}
$$

verwendet [8]. Dabei sind: 


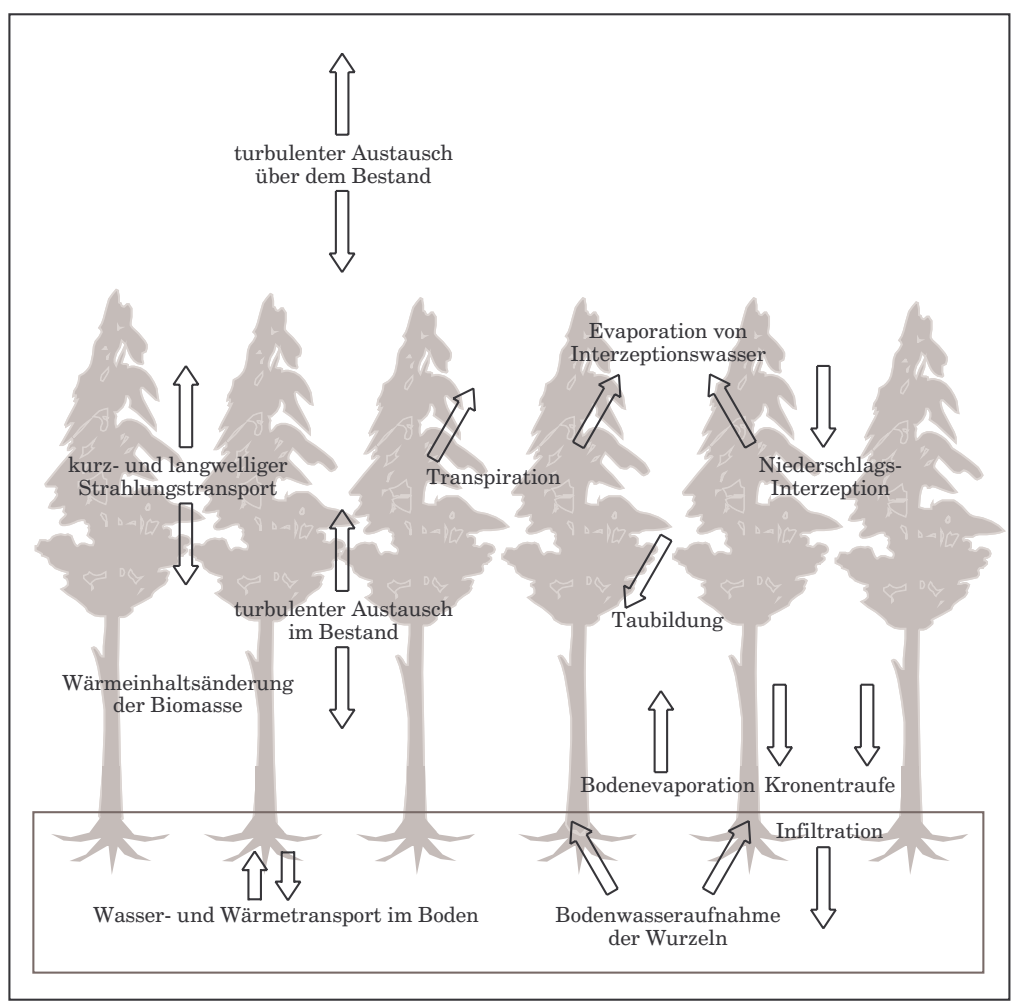

Abbildung 5.4: Überblick über die wichtigsten physikalischen Prozesse, welche im transilienten Modell mit Baumbestand berücksichtigt werden.

$$
\begin{array}{lll}
P_{0} & - & \text { sensibler Wärmestrom am Erdboden }\left[\mathrm{Wm}^{-2}\right] \\
L E_{0} & - & \text { latenter Wärmestrom am Erdboden }\left[\mathrm{Wm}^{-2}\right] \\
B_{0} & - & \text { Bodenwärmestrom }\left[\mathrm{Wm}^{-2}\right] .
\end{array}
$$

Die linke Seite der Gleichung ist die Nettostrahlungsbilanz am Erdboden $F_{0}$.

Die Temperatur an der Oberfläche des Waldbodens $T_{g}$ wird aus der Energiebilanz der Bodenoberfläche mittels Newton - Raphson - Verfahren zur Nullstellenbestimmung ermittelt. Dabei wird solange iteriert, bis sich die Summe von latentem Wärmefluß $L E_{0}$, sensiblem Wärmefluß $P_{0}$, Bodenwärmestrom $B_{0}$ und Nettostrahlungsbilanz $F_{0}$ nur noch geringfügig von Null unterscheidet. Da analytische Ausdrücke für die Ableitung von sensiblem und latentem Wärmestrom nach $T_{g}$ gewonnen werden müssen, werden für den sensiblen und latenten Wärmestrom Näherungsformeln verwendet, die auch den molekularen Transport in der bodennächsten Schicht berücksichtigen.

Dabei wird angenommen, daß in der untersten Modellschicht gleichzeitig turbulente und molekulare Transporte stattfinden. Zur Berechnung der fühlbaren und latenten Wärmeflüsse in Bodennähe wird deshalb eine Methode angewendet, die beide (turbulente und molekulare) Transporte berücksichtigt [31]. Der 
turbulente Anteil kann nach der Ähnlichkeitstheorie berechnet werden nach:

$$
P_{0}=-\rho c_{p} \kappa u_{*} T_{*}
$$

Dabei ist $u_{*}$ die Schubspannungsgeschwindigkeit, $c_{p}$ die spezifische Entalphie der Luft und $T_{*}$ ein charakteristisches Temperaturmaß gegeben durch:

$$
T_{*}=\left(\Theta\left(z_{1}\right)-\Theta\left(z_{s}\right)\right) / \ln \left(z_{1} / z_{0}\right),
$$

mit $\Theta\left(z_{1}\right)$ als potentielle Temperatur der untersten Modellschicht und $\Theta\left(z_{s}\right)$ als potentielle Temperatur an der Obergrenze der viskosen Unterschicht der Atmosphäre, die sich direkt an die Erdoberfläche anschließt und im allgemeinen nur wenige Millimeter dick ist. Die Gleichung für $P_{0}$ kann unter Benutzung der obengenannten Beziehung auch als

$$
P_{0}=-c_{1}\left(\Theta\left(z_{1}\right)-\Theta\left(z_{s}\right)\right)
$$

geschrieben werden, wobei einige Terme in der Größe $c_{1}$ zusammengefaßt wurden. Da im allgemeinen aber $z_{s}$ und $\Theta\left(z_{s}\right)$ nicht bekannt sind, muß nach einem Weg gesucht werden, diese Größen zu ersetzen. Dafür wird für die Grenzfläche zwischen der viskosen Unterschicht und der darüberliegenden turbulenten Schicht Stetigkeit der vertikalen Flüsse angenommen, eine Voraussetzung, die nach den allgemeinen Erfahrungen erfüllt ist. Der turbulente Fluß wird durch die letztgenannte Beziehung angegeben. Der molekulare Fluß in der viskosen Unterschicht läßt sich darstellen in der Form:

$$
P=-\rho K \frac{\left(T\left(z_{s}\right)-T_{g}\right)}{z_{s}} .
$$

Dabei ist $K$ die molekulare Diffusivität für Wärme, $T\left(z_{s}\right)$ ist die $\Theta\left(z_{s}\right)$ entsprechende Temperatur und $T_{g}$ ist die Temperatur der Erdoberfläche. Diese letzte Gleichung läßt sich auch schreiben in der Form:

$$
P=-c_{2}\left(\Theta\left(z_{s}\right)-\Theta_{g}\right),
$$

wobei in $c_{2}$ wieder einige Größen zusammengefaßt wurden. Durch Umstellen der so erhaltenen Gleichungen für $P_{0}$ und $P$ nach $\Theta\left(z_{s}\right)$, Gleichsetzen und nochmaliges Umstellen erhält man die folgende Beziehung für $P_{0}$ :

$$
P_{0}=-c_{1}\left(\Theta\left(z_{1}\right)-\Theta_{g}\right)\left(1+c_{1} / c_{2}\right)^{-1}
$$

Dabei ergibt sich $c_{1} / c_{2}$ zu:

$$
c_{1} / c_{2}=\frac{c_{p} \kappa^{2} v_{h}\left(z_{1}\right) z_{1}}{K}
$$

Somit kann der fühlbare Wärmefluß in Bodennähe unter Einbeziehung der molekularen Flüsse berechnet werden, ohne die Höhe und die Temperatur an der Obergrenze der viskosen Unterschicht zu kennen.

Eine ähnliche Beziehung ergibt sich für den latenten Wärmefluß in Bodennähe. Hier muß jedoch beachtet werden, daß Wasserdampf in Luft etwa $1.58 \mathrm{mal}$ 
schneller molekular diffundiert als Wärme, so daß die molekulare Diffusivität mit dem Faktor 1.58 multipliziert werden muß. Damit erhält man:

$$
L E_{0}=-c_{1} \frac{m_{w}}{c_{p}}\left(q\left(z_{1}\right)-q_{s, g}\right)\left(1+c_{1} /\left(1.58 c_{2}\right)\right)^{-1} .
$$

Dabei ist $m_{w}$ ein Faktor, der die Feuchteverfügbarkeit an der Bodenoberfläche beschreibt, $q\left(z_{1}\right)$ ist die spezifische Feuchte in der untersten Modellschicht und $q_{s, g}$ ist die Sättigungsfeuchte der Luft an der Bodenoberfläche.

\subsection{Lösung der Wärmeleitungsgleichung im Boden}

Im Bodentemperaturmodell wird die Wärmeleitungsgleichung im Boden gelöst:

$$
C_{b} \frac{\partial T_{b}}{\partial t}=-\frac{\partial}{\partial z}\left(\lambda_{b} \frac{\partial T_{b}}{\partial z_{b}}\right) \text {. }
$$

Dabei ist $z_{b}$ die Bodentiefe, $T_{b}$ die Bodentemperatur und $C_{b}$ die volumetrische Wärmekapazität der Bodenschichten in $\mathrm{Jm}^{-3} \mathrm{~K}^{-1}$. Sie ergibt sich durch Multiplikation der spezifischen Wärmekapazität des Bodens mit der Bodendichte. $\lambda_{b}$ ist die Wärmeleitfähigkeit der Bodenschichten. Für die Werte von $C_{b}$ und $\lambda_{b}$ werden von der Zusammensetzung, insbesondere vom Wassergehalt der einzelnen Bodenschichten bestimmt.

Die volumetrische Wärmekapazität $C_{b}$ setzt sich aus den volumetrischen Wärmekapazitäten der Bodenkompartimente, gewichtet mit den Volumenanteilen zusammen. Unter Vernachlässigung der Wärmekapazität der Bodenluft ergibt sich somit:

$$
C_{b}=\rho_{m} \Theta_{m, f} c_{m}+\rho_{o} \Theta_{o, f} c_{o}+\rho_{w} \Theta c_{w} .
$$

Dabei sind $c_{m}, c_{o}, c_{w}, c_{l}$ die spezifischen Wärmekapazitäten des mineralischen Anteils, des Anteils an organischem Materials am Boden sowie des Wassers. Die Größen $\rho$. sind die Dichten, $\Theta$. . bezeichnen die Volumenanteile der einzelnen Kompartimente am feuchten Boden, mit der Ausnahme, daß das alleinstehende $\Theta$ den volumetrischen Bodenwassergehalt bezeichnet. Da nur die Meßwerte des mineralischen und des organischen Volumenanteils am trockenen Boden $\Theta_{m}$ und $\Theta_{0}$ vorliegen, mußte die obige Gleichung in etwas abgewandelter Form angewendet werden, um die unterschiedlichen Volumenanteile der festen Bodenkompartimente je nach Wassergehalt zu berücksichtigen:

$$
C_{b}=(1-\Theta)\left(\rho_{m} \Theta_{m} c_{m}+\rho_{o} \Theta_{o} c_{o}\right)+\Theta \rho_{w} c_{w} .
$$

Die spezifischen Wärmekapazitäten der einzelnen Kompartimente sind unabhängig von der Bodentiefe, so daß die volumetrische Wärmekapazität nur durch die unterschiedlichen Volumenanteile mit der Bodentiefe variiert. Für die Wärmeleitfähigkeit kann eine ähnliche Gleichung aufgestellt werden, nur wurde hier aufgrund fehlender Angaben über die Wärmeleitfähigkeiten der festen Kompartimente eine aus Bodentemperaturmessungen und Simulationsrechnungen bestimmte empirische Beziehung angewendet ${ }^{3}$ :

$$
\lambda_{b}=a \frac{\Theta}{\Theta_{s}}+\lambda_{r} .
$$

\footnotetext{
${ }^{3}$ Oltchev 1998: persönliche Mitteilung
} 


\begin{tabular}{lllcc}
\hline \hline Bodentiefe $[\mathrm{m}]$ & $\Theta_{m}\left[\mathrm{~m}^{3} \mathrm{~m}^{-3}\right]^{*}$ & $\Theta_{\circ}\left[\mathrm{m}^{3} \mathrm{~m}^{-3}\right]^{*}$ & $a\left[\mathrm{Wm}^{-1} \mathrm{~K}^{-1}\right] \oplus$ & $\lambda_{r}\left[\mathrm{Wm}^{-1} \mathrm{~K}^{-1}\right] \oplus$ \\
\hline $0.0 \rightarrow 0.2$ & 0.600 & 0.400 & 1.22 & 0.140 \\
$0.2 \rightarrow 0.6$ & 0.800 & 0.200 & 1.35 & 0.200 \\
$0.6 \rightarrow 1.4$ & 0.990 & 0.010 & 1.35 & 0.200 \\
$1.4 \rightarrow 3.0$ & 1.000 & 0.000 & 1.35 & 0.200 \\
\hline \hline
\end{tabular}

Tabelle 5.3: Zur Modellierung der Bodentemperaturen benutzte Parameter (Quellen: * - [10], $\oplus$ Oltchev (persönliche Mitteilung)). Es bedeuten: $\Theta_{m}$ - Volumenanteil an mineralischem Material, $\Theta_{0}$ - Volumenanteil an organischem Material, $a$ - empirische Konstante, $\lambda_{r}$ mittlere Wärmeleitfähigkeit bei absoluter Trockenheit.

Dabei ist $\Theta_{s}$ der volumetrische Wassergehalt bei Wassersättigung, $a$ eine empirische Konstante und $\lambda_{r}$ die mittlere Wärmeleitfähigkeit der restlichen Bodenanteile.

Der Vollständigkeit halber seien hier nur die Volumenanteile am trockenen Boden und die Parameter der empirischen Beziehung zur Bestimmung der Wärmeleitfähigkeit in den unterschiedlichen Bodenschichten aufgeführt (siehe Tab. 5.3).

\subsection{Bodenfeuchtemodellierung}

Das einfache vertikal aufgelöste Bodenfeuchtemodell löst die Kontinuitätsgleichung für den eindimensionalen vertikalen Bodenwassertransport mit Quellbzw. Senkentermen für jede Bodenschicht.

Eine gute Übersicht über die Gesetzmäßigkeiten, denen die Bodenfeuchte unterliegt, findet sich in [14] und [15]. Ausführlichere Informationen liefert [33].

Die zeitliche Änderung des volumetrischen Bodenwassergehaltes $\Theta_{k}$ (Volumen Bodenwasser pro Einheitsvolumen feuchter Boden) einer Bodenschicht $k$ wird auf der Grundlage der eindimensionalen Kontinuitätsgleichung aus den vertikalen Bodenwasserflüssen berechnet. Genau wie im atmosphärischen Teil des Modells wird auch hier Homogenität in der Horizontalen angenommen. Demnach ergibt sich unter Berücksichtigung eines Quell- oder Senkenterms $S_{k}$ in der Schicht $k$ die folgende Beziehung zur Berechnung der zeitlichen Änderung des volumetrischen Bodenwassergehaltes in der Schicht $k$ :

$$
\frac{\partial \Theta_{k}}{\partial t}=-\frac{\partial Q_{k}}{\partial z}-S_{k}
$$

wobei $Q_{k}$ der Bodenwasserfluß durch die Obergrenze der Schicht $k$ ist. Als Quelle wird für die oberste Bodenschicht die effektive Niederschlagsrate am Boden (siehe Abschnitt 5.10), als Senke die Evaporation von der Bodenoberfläche berücksichtigt. Eine weitere Senke für die Bodenschichten stellt die Wasseraufnahme der Wurzeln infolge der Transpiration der Bäume dar, sofern Wurzeln in der jeweiligen Schicht vorhanden sind.

Für das Verständnis der Wassertransporte in Böden sehr nützlich ist das Konzept des Potentials. Das Potential ist bezüglich des Bodenwassers definiert als die Arbeit, die notwendig ist, um eine Einheitsmenge (Volumen, Masse oder Gewicht) Wasser von einem gegebenen Punkt eines Kraftfeldes zu einem Be- 
zugspunkt zu transportieren [33]. Aufgrund dieser Definition des Potentials ist das Bodenwasser ähnlich wie ein Körper im Gravitationsfeld der Erde immer „bestrebt" , von einem Ort höheren Potentials (= spezifische Energie) zu einem Ort niederen Potentials zu fließen. Für die Beschreibung der vertikalen Bodenwasserflüsse ist also das vertikale Energiegefälle des im Boden gespeicherten Wassers entscheidend. Das Gesamtpotential des Bodenwassers setzt sich aus mehreren Teilpotentialen zusammen [33], die hier nicht ausführlich diskutiert werden sollen. Für die meisten Anwendungen ist die Beschränkung auf 2 Teilpotentiale ausreichend: das Gravitationspotential (entspricht dem Ort des Wassers im Gravitationsfeld der Erde) und das Matrixpotential (Maß für die potentielle Energie der Wasserbindung in den Bodenkapillaren). Aus diesem Grunde beschränkt sich auch dieses Modell auf diese beiden Potentiale, deren Summe auch als hydraulisches Potential bezeichnet wird. Wird das hydraulische Potential definiert als zu verrichtende Arbeit bezogen auf ein Einheitsvolumen $\left(1 \mathrm{~m}^{3}\right)$, so erhält man für dafür die Einheit des Druckes (hPa); bezogen auf ein Einheitsgewicht ( $1 \mathrm{~N}=1 \mathrm{~kg} \mathrm{~m} \mathrm{~s}^{-2}$ ) erhält man die Einheit der Länge (m Wassersäule). Dies sind die am häufigsten verwendeten Einheiten für das hydraulische Potential. In den hier beschriebenen Beziehungen muß das hydraulische Potential in der Einheit m verwendet werden. Der bei der Angabe in $\mathrm{hPa}$ angegebene Wert entspricht dem Druck auf der Grundfläche einer Wassersäule einer bestimmten Höhe, welche dem Potential in m entspricht. Demnach ergibt sich für die „Umrechnung“ die Beziehung: $1000 \mathrm{hPa} \hat{=} 10 \mathrm{~m} \rightarrow 1 \mathrm{hPa} \hat{=} 10^{-2} \mathrm{~m}$.

Der vertikale Fluß $Q_{k}$ des Bodenwassers durch die untere Begrenzung einer Bodenschicht k ist entsprechend dem Darcy'schen Gesetz [14] definiert nach:

$$
Q_{k}=-K_{k} \frac{\partial H_{k}}{\partial z},
$$

wobei $K_{k}$ die hydraulische Leitfähigkeit ist, und $H_{k}$ ist das Potential. Unter Aufspaltung von $H_{k}$ in Gravitations- und Bodenwasserpotential $\Psi_{k}$ läßt sich $Q_{k}$ auch ausdrücken mit:

$$
Q_{k}=-K_{k}\left(\frac{\partial \Psi_{k}}{\partial z}-1\right)
$$

Die Werte für das Bodenwasserpotential werden im Bodenmodell berechnet nach der empirischen Formel von Clapp \& Hornberger, genannt in [35]:

$$
\Psi_{k}=\Psi_{s, k}\left(\frac{\Theta_{k}}{\Theta_{s, k}}\right)^{-B_{k}} .
$$

Dabei ist $\Psi_{s, k}$ das Bodenwasserpotential der Bodenschicht $k$ bei Wassersättigung, $\Theta_{k}$, Einheit: [1], ist der volumetrische Bodenwassergehalt der Schicht $k$ und $\Theta_{s, k}$, Einheit: [1], ist der volumetrische Bodenwassergehalt der Schicht $k$ bei Wassersättigung. $B_{k}$, Einheit: [1], ist ein empirischer Parameter, der von der Textur (Korngrößenzusammensetzung) des Bodens abhängt. Das Bodenwasserpotential bei Wassersättigung zählt zu den hydraulischen Eigenschaften eines Bodens und muß durch Messungen bestimmt werden. Durch Einsetzen der Gleichung 5.16 in die Beziehung für $\frac{\partial \Theta_{k}}{\partial t}$ erhält man eine inhomogene parabolische Differentialgleichung zweiter Ordnung. Diese Differentialgleichung ist aufgrund der Abhängigkeit der hydraulischen Leitfähigkeit und des Matrixpotentials vom volumetrischen Wassergehalt nichtlinear, und kann nur numerisch gelöst werden. Der Boden im Modellgebiet läßt sich in 5 Bereiche mit 


\begin{tabular}{lllcl}
\hline \hline Bodentiefe $[\mathrm{m}]$ & $\Theta_{s}\left[\mathrm{~m}^{2} \mathrm{~m}^{-2}\right]$ & $K_{s}\left[\mathrm{~m} s^{-1}\right]$ & $B[1]$ & $\Psi_{s}[\mathrm{~m}]$ \\
\hline $0.0 \rightarrow 0.2$ & 0.451 & $1.158 \cdot 10^{-5}$ & 5.39 & -0.146 \\
$0.2 \rightarrow 0.6$ & 0.451 & $2.917 \cdot 10^{-5}$ & 5.39 & -0.146 \\
$0.6 \rightarrow 1.4$ & 0.485 & $5.833 \cdot 10^{-7}$ & 5.30 & -0.566 \\
$1.4 \rightarrow 3.0$ & 0.451 & $1.333 \cdot 10^{-8}$ & 5.39 & -0.146 \\
\hline \hline
\end{tabular}

Tabelle 5.4: Zur Modellierung der Bodenfeuchte benutzte Parameter (Quelle: [10]): $\Theta_{s}=$ volumetrischer Bodenwassergehalt bei Sättigung, $K_{s}=$ hydraulische Leitfähigkeit bei Sättigung, $B=$ empirische Konstante, $\Psi_{s}=$ Bodenwasserpotential bei Sättigung.

unterschiedlichen hydraulischen Eigenschaften einteilen. Die Werte der Parameter für diese Bereiche sind in Tabelle 5.4 zusammengefaßt. Die Bodenschichtung für den betrachteten Waldstandort (Versuchsfläche Solling F1) wird beschrieben von Bredemeier \& Tiktak 1993, genannt in [10]. Die benötigten Parameter für die Bodenschichten wurden mittels statistischem Verfahren von Clapp \& Hornberger 1978, genannt in [10], aus der Textur der Bodenschichten abgeleitet.

\subsection{Berechnung der Strahlungsflüsse im Bestand}

Die kurzwelligen und langwelligen Strahlungsflüsse im Bestand werden zur Berechnung der Erdoberflächentemperatur und der Temperatur der Baumoberfläche benötigt. Es wird dabei ein sehr einfaches Strahlungsmodell verwendet. Das gesamte Spektrum wird für die Simulation in 2 Teile aufgeteilt, die getrennt parametrisiert werden: die kurzwellige Strahlung - sie wird von der sichtbaren Strahlung (Licht) zusammen mit der photosynthetisch aktiven Strahlung (PAR) gebildet (siehe auch Abschnitt 5.2) - und die langwellige Strahlung (Wärmestrahlung).

Über dem Bestand werden der abwärtsgerichtete kurzwellige Strahlungsfluß $F_{s, h}^{\downarrow}$ (Globalstrahlung) und der abwärtsgerichtete langwellige Strahlungsfluß (langwellige atmosphärische Gegenstrahlung) $F_{l, h}^{\downarrow}$ aus Meßwerten vorgegeben. Die Verwendung von Strahlungsmeßwerten innerhalb des Bestandes (Meßhöhe von $2 \mathrm{~m}$ ) gestaltet sich schwierig, da für die Berechnung der Baumoberflächentemperatur in den einzelnen Bestandsschichten die Vertikalprofile der kurz- und langwelligen Strahlungsbilanz bekannt sein müssen. Deshalb wurden nur die Meßwerte an der Bestandesobergrenze vorgegeben und die Vertikalprofile im Bestand berechnet.

Bei der Berechnung der Strahlungsverteilung im Bestand muß berücksichtigt werden, daß zwischen den Bäumen Lücken bestehen, in denen die lang- und kurzwelligen Strahlungsflüsse durch die Vegetation nicht beeinflußt werden. Die Größe dieser Lücken wird durch den sogenannten Überdeckungsgrad mit Vegetation $n_{w}$ beschrieben. Dieser Parameter ergibt sich aus Vergleichen der gemessener Strahlungswerte am Waldboden mit den zur selben Zeit über nicht vegetationsüberdecktem Boden gemessenen Werten. In diesem Modell wird ein Wert von $n_{w}=0.8$ verwendet.

Die kurzwellige Strahlungsbilanz an der Bestandsobergrenze $F_{s, h}$ berechnet 
sich durch Subtraktion der reflektierten kurzwelligen Strahlung von $F_{s, h}^{\downarrow}$ :

$$
F_{s, h}=\left(1-\alpha_{w}\right) F_{s, h}^{\downarrow} .
$$

$\alpha_{w}$ ist die kurzwellige Albedo der Baumoberfläche. Im Modell wird ein Wert von $\alpha_{w}=0.1$ verwendet.

Die so berechnete Strahlungsbilanz wäre für einen sehr dichten Bestand richtig $\left(n_{w}=1\right)$, beschreibt in unserem Falle eines weniger dichten Bestandes aber nur die Strahlungsbilanz an der Obergrenze eines Baumes. Für den gesamten Bestand muß die so berechnete kurzwellige Strahlungsbilanz unter der Voraussetzung der horizontalen Homogenität der geometrischen Parameter der Bäume und des Bestandes noch mit $n_{w}$ multipliziert werden. Die Unterscheidung zwischen Einzelbaum und Bestand braucht aber vor dem Hintergrund der Berechnung des Energie- und Stoffaustausches zwischen der Baumoberfläche und der Umgebungsluft nicht getroffen zu werden, da hier nur die Strahlungsbeeinflussung im Bereich der von der Vegetation eingenommenen Fläche jeder Modellschicht eine Rolle spielt (Annahme: die in einer Schicht von der Biomasse absorbierte Strahlungsenergie geht vollständig in die Erwärmung der Baumoberfläche und in die für die Evapotranspiration notwendige Energie). Deshalb wird im folgenden nur die Parametrisierung der vertikalen Strahlungsflüsse in einem sehr dichten Bestand $\left(n_{w}=1\right)$ beschrieben. Eine Unterscheidung zwischen Einzelbaum und Bestand braucht erst wieder vor dem Hintergrund der Berechnung der Erdoberflächentemperatur aus der Energiebilanz am Boden getroffen zu werden.

Die kurzwellige Strahlungsbilanz $F_{s, k}$ in einer Schicht $k$ innerhalb des Bestandes kann in Anlehnung an das Lambert-Bougetsche Gesetz der Extinktion mittels einer Exponentialfunktion in Abhängigkeit von der über dem betrachteten Niveau befindlichen rel. Baumoberfläche berechnet werden:

$$
F_{s, k}=F_{s, h} \exp \left(-k_{e x t} L\left(z_{k}\right)\right) .
$$

$L\left(z_{k}\right)$ ist die gesamte Vegetationsoberfläche zwischen der Schicht $k$ und der Obergrenze des Baumes. $k_{e x t}$ ist ein „Extinktionsparameter" , der sich aus dem zeitlich konstanten Extinktionskoeffizienten für kurzwellige Strahlung und einem additiven Zusatz, welcher die je nach Tages- und Jahreszeit unterschiedliche Weglänge, die das Licht durch den Kronenraum zurücklegt, berücksichtigt:

$$
k_{e x t}=0.2\left[\frac{1}{\cos (\zeta)}-1\right]+k_{0} .
$$

Dabei ist $k_{0}$ der Extinktionskoeffizient für die direkte kurzwellige Strahlung. Für ihn wird ein Wert von 0.5 verwendet. Der Winkel $\zeta$ ist die Zenitdistanz, also der Winkel unter dem die direkte kurzwellige Sonnenstrahlung auf die durch die Obergrenze des Bestandes gebildete Ebene auftrifft. Der Kosinus der Zenitdistanz wird berechnet nach [22]:

$$
\cos (\zeta)=\sin (\phi) \sin (\delta)+\cos (\phi) \cos (\delta) \cos (\tau) \text {. }
$$

$\phi$ ist die geographische Breite, $\delta$ die Deklination und $\tau$ der Stundenwinkel der Sonne. Die geringe Neigung der Erdoberfläche des betrachteten Gebietes gegenüber der Horizontalen wird dabei vernachlässigt. Da die Deklination der 
Sonne sich im Laufe eines Tages nur geringfügig ändert, wird für diese während eines Tages ein konstanter Wert verwendet, der jedoch für jeden Tag neu berechnet wird.

Die Beziehung für $k_{e x t}$ gilt streng genommen nur für den direkten Strahlungsanteil der Globalstrahlung. Der diffuse Strahlungsanteil verhält sich dagegen anders. Die diffuse Strahlung spielt eine große Rolle bei starker Bewölkung und zur Zeit vor und nach Sonnenaufgang sowie Sonnenuntergang. Das macht sich in den Meßwerten der Globalstrahlung in $2 \mathrm{~m}$ Höhe innerhalb des Bestandes dadurch bemerkbar, daß schon kurz nach Sonnenaufgang Werte größer als Null gemessen werden, obwohl nach der Beziehung für $k_{e x t}$ noch keine direkte Strahlung am Waldboden vorhanden sein sollte. Diese Beobachtung kann nur mit dem Vorhandensein der diffusen Strahlung erklärt werden. Um dennoch die diffuse kurzwellige Strahlung nicht in einem extra Strahlungsmodell berechnen zu müssen, wurde die Berechnung von $k_{e x t}$ mit einem eigenen Ansatz modifiziert. Es hat sich gezeigt, daß es ausreicht, den Wert für $k_{e x t}$ nach oben zur begrenzen, um zumindest die Wirkung der diffusen Strahlung zu Zeiten des Sonnenauf- und -untergangs zu berücksichtigen. Nach eigenen Untersuchungen hat sich dabei eine obere Grenze von 0.5 für $k_{e x t}$ bewährt.

Bei der langwelligen Strahlung muß neben der atmosphärischen Gegenstrahlung über dem Bestand, deren Aufnahme und teilweiser Abstrahlung von der Bestandsobergrenze nach oben, sowie der Extinktion beim Eindringen in den Bestand auch noch die langwellige Ausstrahlung der Biomasse des Bestandes und des Erdbodens berücksichtigt werden. Für die langwellige Ausstrahlung des Bestandes wird der Kronenraum als eine einzige abstrahlende Schicht aufgefaßt. Für die Temperatur $T_{v e g}$ dieser Schicht wird die mit der Baumoberflächendichte $A_{\text {tree }}$ (siehe Kap. 4) jeder Bestandsschicht gewichtete Mitteltemperatur des Bestandes verwendet:

$$
T_{\text {veg }}=\frac{\sum_{i=1}^{N} A_{\text {tree }, k} T_{w, k}}{\sum_{i=1}^{N} A_{\text {tree }, k}} .
$$

Die langwellige Ausstrahlung $A_{b}$ des Bestandes berechnet sich demnach nach dem für einen nicht ideal schwarzen Körper modifizierten Gesetz von Stefan und Boltzmann folgendermaßen:

$$
A_{b}=\epsilon_{w} \sigma T_{v e g}^{4} .
$$

$\epsilon_{w}$ ist der langwellige Emissionskoeffizient des Fichtenbestandes, er wird mit $\epsilon_{w}=0.98$ angesetzt [45]. Es wird angenommen, daß die Wärmeabstrahlung nach allen Seiten gleichmäßig erfolgt, d.h. nach oben und nach unten wird dieselbe Energiemenge abgestrahlt.

Das Profil der langwelligen Strahlungsbilanz im Bestand wird nicht explizit berechnet. Stattdessen wird direkt das benötigte Profil der Gesamtstrahlungsbilanz (kurzwellig und langwellig) mittels einer empirischen Formel nach Impens und Lemeur (1969), genannt in [16] und [26]:

$$
R_{n v e g}\left(z_{k}\right)=R_{n v e g}\left(h_{p}\right) \exp \left[-k_{1} L_{v}\left(z_{k}\right)+k_{2} L_{v}\left(z_{k}\right)^{2}\right]
$$


berechnet. Dabei ist $R_{\text {nveg }}\left(h_{p}\right)$ die Gesamtstrahlungsbilanz an der Bestandsobergrenze und $k_{1}=0.622$ sowie $k_{2}=0.0553$ sind empirisch gewonnene Koeffizienten nach Impens und Lemeur (1969). Mit dieser Beziehung erhält man das Profil der Gesamtstrahlungsbilanz für den vegetationsüberdeckten und den nicht vegetationsüberdeckten Anteil, was bei der Aufstellung der Energiebilanz zur Berechnung der Vegetationsoberflächentemperatur berücksichtigt werden muß, da für die Erwärmung der Vegetationsoberfläche nur der Anteil der vegetationsüberdeckten Fläche an der Gesamtstrahlungsbilanz in Frage kommt. Die Verwendung einer nur für den vegetationsüberdeckten Anteil gültigen Beziehung nach Yamada [46]:

$$
R_{n v e g}\left(z_{k}\right)=R_{n v e g}\left(h_{p}\right)\left[\exp \left[-k_{n} L_{v}\left(z_{k}\right)\right]-n_{w}\left(1-\frac{z_{k}}{h_{p}}\right) \exp \left[-k_{n} L A I\right]\right],
$$

mit $k_{n}$ als Extinktionskoeffizienten für die Gesamtstrahlungsbilanz $\left(k_{n}=0.6\right)$ hat sich dagegen nicht bewährt. Die benötigte Gesamtstrahlungsbilanz an der Bestandsobergrenze wird berechnet nach Deardorff 1978 [8]:

$$
R_{n v e g}\left(h_{p}\right)=F_{s, h}^{\downarrow}+\epsilon_{w} F_{l, h}^{\downarrow}-\epsilon_{w} \sigma T_{v e g}^{4},
$$

wobei $F_{s, h}^{\downarrow}$ der kurzwellige und $F_{l, h}^{\downarrow}$ der langwellige abwärtsgerichtete Strahlungsfluß über dem Bestand ist.

Die für die Berechnung der Vegetationsoberflächentemperatur in jeder Bestandsschicht nötige Kenntnis der vertikalen Änderung der Gesamtstrahlungsbilanz ergibt sich durch analytische Ableitung des Ausdruckes für $R_{n v e g}\left(z_{k}\right)$ nach Gleichung 5.24. Dabei muß von der Beziehung zwischen der Vegetationsoberfläche und der Vegetationsoberflächendichte in der Schicht $k$ Gebrauch gemacht werden:

$$
A_{\text {tree }, k}=\frac{\partial L_{v}\left(z_{k}\right)}{\partial z} .
$$

Für die Berechnung der Erdoberflächentemperatur werden der abwärtsgerichtete kurzwellige Strahlungsfluß $F_{s, 0}^{\downarrow}$ und der abwärtsgerichtete langwellige Strahlungsfluß $F_{l, 0}^{\downarrow}$ benötigt. Wie oben angegeben, muß hier der Überdeckungsgrad mit Vegetation $n_{w}$ berücksichtigt werden. Der kurzwellige abwärtsgerichtete Strahlungsfluß $F_{s, 0}$ wird im Modell jedoch nicht explizit berechnet, sondern es kann unter Benutzung von Gl. 5.19 direkt die kurzwellige Strahlungsbilanz $F_{s, 0}$ am Boden berechnet werden. Unter Beachtung der Reflektion der kurzwelligen Strahlung an der Bodenoberfläche ergibt sich:

$$
F_{s, 0}=\left(1-\alpha_{g}\right) F_{s, h} \exp \left(-k_{e x t} L A I\right),
$$

wobei $\alpha_{g}$ die kurzwellige Albedo der Bodenoberfläche ist. Im Modell wird eine Wert von $\alpha_{g}=0.1$ verwendet [45].

Für den abwärtsgerichteten langwelligen Strahlungsfluß an der Bodenoberfläche wird eine Beziehung nach [8] verwendet:

$$
F_{l, 0}^{\downarrow}=\left(1-n_{w}\right) F_{l, h}^{\downarrow}+n_{w} \frac{\epsilon_{w} \sigma T_{v e g}^{4}+\left(1-\epsilon_{w}\right) \epsilon_{g} \sigma T_{g}^{4}}{\epsilon_{w}+\epsilon_{g}-\epsilon_{w} \epsilon_{g}} .
$$

Dabei sind $\epsilon_{g}$ und $T_{g}$ der langwellige Emissionskoeffizient bzw. die Temperatur der Bodenoberfläche. Für $\epsilon_{g}$ wird ein Wert von 0.93 verwendet [45]. 
Der für die langwellige Strahlungsbilanz am Boden benötigte, vom Boden ausgehende, langwellige aufwärtsgerichtete Strahlungsfluß $F_{l, 0}^{\dagger}$ wird ebenfalls nach [8] berechnet mit:

$$
\begin{gathered}
F_{l, 0}^{\uparrow}=\left(1-n_{w}\right)\left(\epsilon_{g} \sigma T_{g}^{4}+\left(1-\epsilon_{g}\right) F_{l, h}^{\downarrow}\right) \\
+\frac{n_{w}\left(\epsilon_{g} \sigma T_{g}^{4}+\left(1-\epsilon_{g}\right) \epsilon_{w} \sigma T_{v e g}^{4}\right)}{\epsilon_{w}+\epsilon_{g}-\epsilon_{w} \epsilon_{g}} .
\end{gathered}
$$

\subsection{Energieaustausch zwischen der Vegetation und der Bestandsluft}

Zwischen der Oberfläche der Vegetation und der Umgebungsluft findet ein Austausch sensibler und latenter Wärme statt, der in einem atmosph. Grenzschichtmodell zur Simulation der meteorologischen Verhältnisse im Bestand berücksichtigt werden muß. Für die Berechnung des Uberganges sensibler Wärme von der Baumoberfläche in die umgebende Luft in einer Modellschicht $k$ (fühlbarer Wärmefluß $S_{k}$ ) wird die folgende Beziehung verwendet:

$$
S_{k}=\rho c_{p} m_{b} \frac{\left(T_{w, k}-T_{k}\right)}{r_{b, k}} .
$$

Dabei ist $\rho$ die Luftdichte, $c_{p}$ ist die spezifische Enthalpie der Luft, $T_{w, k}$ ist die Temperatur der Baumoberfläche und $T_{k}$ die Lufttemperatur in der Modellschicht $k . r_{b}$ ist der aerodynamische Übergangswiderstand für den Transport sensibler Wärme durch die Grenzschicht über der Vegetationsoberfläche. Der Faktor $m_{b}$ berücksichtigt den Unterschied zwischen der tatsächlichen Oberfläche der Nadeln und deren horizontaler Projektion. Dieser Unterschied muß hier beachtet werden, da die Beziehung für $S_{k}$ in die Energiebilanzgleichung der Vegetationsoberfläche in den Modellschichten im Bestand eingeht. Dabei ist für die Extinktion der einfallenden Strahlung nur die horizontale Projektion der Nadeloberfläche relevant, während der Wärme- und Feuchteaustausch über die gesamte Oberfläche der Nadeln erfolgt. Für diesen Faktor wurde von Riederer et al. (1988), genannt in [25], ein mittlerer Wert von $m_{b}=2.74$ angegeben, der auch in diesem Modell Verwendung findet.

Für den Übergang von latenter Wärme unter Berücksichtigung der Verdunstung von durch Interzeption festgehaltenem Niederschlagswasser wird die folgende Beziehung verwendet:

$$
L E_{k}=\frac{\rho c_{p}}{\gamma_{k}} m_{b}\left(e^{*}\left(T_{w, k}\right)-e_{k}\right)\left(\frac{N_{k}}{1.58 r_{b, k}}+\frac{1-N_{k}}{1.58 r_{b, k}+r_{s, k}}\right) .
$$

Dabei ist $L$ die spezifische VerdampfungsWärme von Wasser, $\gamma_{k}$ ist die Psychrometerkonstante in der Schicht $k, e^{*}\left(T_{w, k}\right)$ ist der Sättigungsdampfdruck an der Vegationsoberfläche der Schicht $k, e_{k}$ ist der Dampfdruck der Luft und $r_{s, k}$ der Stomatawiderstand in der Schicht $k$. Die Größe $N_{k}$ ist der Anteil der Baumoberfläche in der Schicht $k$, der von Interzeptionswasser eingenommen wird. Über der Fläche dieses Anteils tritt keine Transpiration, sondern nur die Evaporation des Interzeptionswassers auf. Deshalb braucht im ersten Term der 
letzten Klammer nur der aerodynamische Übergangswiderstand berücksichtigt werden.

Der aerodynamische Übergangswiderstand $r_{b}$ muß hier mit dem Faktor 1.58 multipliziert werden, da die Feuchte aufgrund des gegenüber trockener Luft geringeren Molekulargewichtes etwa 1.58 mal so schnell molekular diffundiert, wie die fühlbare Wärme.

Desweiteren wird vereinfachend angenommen, daß die Temperaturen des trockenen und des feuchten Anteils der Baumoberfläche gleich sind. Es gibt eine Anzahl von Argumenten, die dafür sprechen, daß ein solcher Ansatz besser ist, als eine getrennte Berechnung der Temperaturen für den feuchten und den trockenen Anteil der Baumoberfläche (Hancock et al. 1983 genannt in [35]). Dieses Vorgehen läßt sich begründen mit dem Argument, daß die Wärmeenergie zwischen den feuchten und trockenen Anteilen der Vegetation infolge Wärmeleitung und Wärmetransport in der laminaren Grenzschicht der Nadeln effizient ausgetauscht wird.

Der Sättigungsdampfdruck an der Vegetationsoberläche in der Schicht $k$ wird in Abhängigkeit von der Oberflächentemperatur berechnet mit Hilfe der TetensFormel:

$$
e^{*}\left(T_{w, k}\right)=6.1078 \exp \left(\frac{T_{w, k}-273.15 \mathrm{~K}}{T_{w, k}-33.9 \mathrm{~K}}\right) \quad[\mathrm{hPa}] .
$$

Die Psychrometerkonstante berechnet sich nach der Beziehung:

$$
\gamma_{k}=\frac{c_{p}\left(z_{k}\right)}{\frac{M_{w}}{M_{a}} L} .
$$

$p\left(z_{k}\right)$ ist der Luftdruck in der Schicht $k, M_{w}$ ist die molare Masse von Wasser und $M_{a}$ ist die molare Masse trockener Luft. Dabei wird die Abhängigkeit der spezifischen Enthalpie der Luft von der spezifischen Feuchte $q$ berücksichtigt nach:

$$
c_{p}=c_{p, d}(1+0.84 q)
$$

$c_{p, d}$ ist die spezifische Enthalpie für trockene Luft. Diese Gleichung ergibt sich aus den allgemeinen Gesetzen für ein Gemisch zweier als ideal angenommener Gase (trockene Luft und Wasserdampf). Auch die Abhängigkeit der VerdampfungsWärme des Wassers von der Temperatur wird berücksichtigt:

$$
L=1.92 \cdot 10^{6} \cdot\left(\frac{T_{k}}{T_{k}-33.9 \mathrm{~K}}\right)^{2} .
$$

Der aerodynamische Widerstand $r_{b, k}$ entsteht durch die molekulare Grenzschicht, die sich unmittelbar an die Oberfläche der Biomasse anschließt. Der Widerstand $r_{b, k}$ läßt sich in Abhängigkeit vom Gradienten der Teilchenbewegung tangential zur Oberfläche der Biomasse beschreiben. Er kann somit als abhängig von der Windgeschwindigkeit in der Schicht $k$ beschrieben werden, da aufgrund der Haftbedingung die tangentiale Geschwindigkeitskomponente direkt an der Oberfläche verschwindet. Für die Berechnung des aerodynamischen Widerstandes wird die folgende Beziehung nach Norman 1979, genannt bei [18] verwendet:

$$
\frac{1}{r_{b, k}}=180 \sqrt{\frac{l_{b}}{v_{k}}}
$$


Dabei ist $l_{b}$ der durchschnittliche Nadeldurchmesser und $v_{k}$ der Betrag der horizontalen Windgeschwindigkeit in der Schicht $k$. Für $l_{b}$ wird ein Wert von $0.005 \mathrm{~m}$ verwendet [18] (siehe Tab. 5.2).

Der stomatäre Widerstand wird in Anlehnung an Jarvis 1976 [19] berechnet. Die Abhängigkeit der stomatären Leitfähigkeit von der photosynthetisch aktiven Strahlung (PAR), der Blattemperatur $T_{w}$, des Wasserdampfdefizits $\Delta_{a}$ der Luft (Differenz des aktuellen Dampfdrucks zum Sättigungsdampfdruck) und des Nadelwasserpotentials $\Psi_{w, k}$ kann in einer Gleichung zusammengefaßt werden:

$$
\frac{1}{r_{s, k}}=\frac{1}{r_{s, \min }} \cdot f_{1, k}(P A R) \cdot f_{2, k}\left(T_{w, k}\right) \cdot f_{3, k}\left(\Delta_{a, k}\right) \cdot f_{4, k}\left(\Psi_{w, k}\right) .
$$

Dabei ist $r_{s, \min }$ der minimale Stomatawiderstand, der erreicht wird, wenn die Spaltöffnungen vollständig geöffnet sind. Die Funktionen $f_{1, k}, \cdots, f_{4, k}$ sind so festgelegt, daß sie nur Werte im Bereich von 0 (keine Transpiration bei schlechten Umweltbedingungen) bis 1 (minimaler Stomatawiderstand bei idealen Umweltbedingungen) annehmen können.

Die Abhängigkeit von der in der Schicht $k$ einfallenden photosynthetisch aktiven Strahlung läßt sich darstellen in Form einer Exponentialfunktion:

$$
f_{1, k}(P A R)=1-\exp \left(-\beta_{s} P A R\right) .
$$

Dabei ist $\beta_{s}$ ein empirisch bestimmter Parameter, der den Anstieg der "LichtAntwort" -Kurve der Photosynthese für $P A R \rightarrow 0$ beschreibt [19]. Aufgrund der Temperaturabhängigkeit der biochemischen Aktivität in den Pflanzenzellen hängt der Feuchte- und Gasaustausch über die Spaltöffnungen auch von der Temperatur der Nadeloberfläche ab. Diese Abhängigkeit wird durch die Funktion $f_{2, k}\left(T_{w, k}\right)$ ausgedrückt:

$$
\begin{gathered}
f_{2, k}\left(T_{w, k}\right)=\frac{\left(T_{w, k}-T_{\min }\right)\left(T_{\max }-T_{w, k}\right)^{h}}{\left(T_{\text {opt }}-T_{\min }\right)\left(T_{\text {max }}-t_{\text {opt }}\right)^{h}}, \\
h=\frac{T_{\text {max }}-T_{\text {opt }}}{T_{\text {opt }}-T_{\min }} .
\end{gathered}
$$

Dabei bestimmen die Temperaturen $T_{\min }$ und $T_{\max }$ die untere bzw. obere Grenze des Temperaturbereiches in dem die Spaltöffnungen geöffnet sind. $T_{\text {opt }}$ ist diejenige Temperatur, bei der die Spaltöffnungen am weitesten geöffnet sind [19]. Nach Untersuchungen von Schulze und Hall 1982 gen. in [29] nimmt der Stomatawiderstand mit zunehmendem Sättigungsdefizit der umgebenden Luft zu. Dieser Effekt läßt sich nach Lohammar (1980) parametrisieren mit:

$$
f_{3, k}\left(\Delta_{a, k}\right)=\frac{1}{1+\alpha \Delta_{a_{k}}} .
$$

Dabei ist $\alpha$ ein empirischer Parameter $\left(\left[\mathrm{mbar}^{-1}\right]\right)$. 
Die Abhängigkeit vom Nadelwasserpotential wird parametrisiert nach Sellers 1989 genannt bei [29]:

$$
f_{4, k}\left(\Psi_{w, k}\right)= \begin{cases}1 & \text { für } \Psi_{w, k}<\Psi_{1} \\ \frac{\Psi_{w, k}-\Psi_{2}}{\Psi_{1}-\Psi_{2}} & \text { für } \Psi_{1} \leq \Psi_{w, k} \leq \Psi_{2} . \\ 0 & \text { für } \Psi_{w, k}>\Psi_{2}\end{cases}
$$

Dabei sind $\Psi_{1}$ und $\Psi_{2}$ die Werte des Nadelwasserpotentials, bei dem die Spaltöffnungen beginnen sich zu schließen bzw. bei dem sie vollständig geschlossen sind. Die für diese Größen verwendeten Werte entstammen [29].

\subsection{Berechnung der Temperatur der Vegetationso- berfläche}

Für die Berechnung der Energieflüsse zwischen der Baumoberfläche und der umgebenden Luft innerhalb des Bestandes ist die Kenntnis der Temperatur der Vegetationsoberfläche notwendig. Diese Temperatur kann aus der Energiebilanz an dieser Oberfläche bestimmt werden. Für eine Modellschicht $k$ innerhalb des Bestandes ergibt sich die Energiebilanz zu:

$$
E_{b, k}=R_{n v e g}\left(z_{k}\right)-m_{b} \epsilon_{w} \sigma T_{w, k}^{4}-L E_{k}-S_{k}+\Delta Q_{k} .
$$

dabei ist $R_{n v e g}\left(z_{k}\right)$ die absorbierte langwellige und kurzwellige Strahlungsbilanz in der Schicht $k$ und $\Delta Q_{k}$ ist die Änderung des Wärmeinhaltes der Vegetation. $\epsilon_{w}$ ist der langwellige Emissionskoeffizient des Fichtenbestandes und $\sigma$ die Stefan-Boltzmann-Konstante. Die Bestimmung des Termes $\Delta Q_{k}$ gestaltet sich aufgrund der mit dem Wassergehalt variierenden Wärmekapazität der Vegetationselemente und der unbekannten Temperaturverteilung im Inneren der Biomasse kompliziert. Die Parametrisierung dieses Terms weist nach Lee und Black (1993) genannt in [18] noch größere Unsicherheiten auf. Hier wurde für diesen Term ein vereinfachter Ansatz gewählt:

$$
\Delta Q_{k}=0.1 c_{h, k} \rho_{h, k} \frac{T_{w, k}(t-\Delta t)-T_{w, k}(t)}{\Delta t} .
$$

Hierbei ist $c_{h, k}$ die spezifische Wärmekapazität des Holzes in der Schicht $\mathrm{k}$ und $\rho_{h, k}$ die entsprechende Dichte. Der Faktor 0.1 berücksichtigt, daß sich im Mittel nur $10 \%$ der Biomasse des Bestandes merklich am Wärmeaustausch mit der Bestandesluft beteiligen ${ }^{4}$.

Für die Berechnung der Vegetationsoberflächentemperatur wird nun angenommen, daß die Energiebilanz in jeder Bestandsschicht geschlossen ist, d.h. die Energie der absorbierten Nettostrahlung verringert um die langwellige Ausstrahlung der Vegetationsschicht, geht vollständig in den sensiblen und latenten Wärmetransport zwischen Baumoberfläche und Umgebungsluft und in die Wärmeinhaltsänderung der Biomasse. Diese Annahme scheint berechtigt,

\footnotetext{
${ }^{4}$ Oltchev 1998: persönliche Mitteilung
} 
ist aber dennoch umstritten. Messungen für den gesamten Bestand zeigen oft Lücken in der Schliesung der Energiebilanz, wobei noch nicht ganz klar ist, ob dies an der unzureichenden Genauigkeit der Messungen oder an einer lückenhaften Beschreibung der die Energiebilanz beeinflussenden Prozesse liegt.

Unter der Voraussetzung einer geschlossenen Energiebilanz kann die Temperatur der Baumoberfläche $T_{w, k}$ in jedem Zeitschritt neu bestimmt werden, indem die Energiebilanz als Funktion von $T_{w, k}$ aufgefaßt wird und die Nullstelle dieser Funktion bestimmt wird.

\subsection{Berechnung des Nadelwasserpotentials}

Für die Berechnung des Stomatawiderstandes wird das Nadelwasserpotential in den einzelnen Schichten des Bestandes benötigt. Das Nadelwasserpotential hat bezüglich des Wassers in der Pflanze dieselbe Bedeutung, wie das hydraulische Potential des Bodenwassers, d.h. es beschreibt den energetischen Zustand des in der Pflanze vorhandenen Wassers. Bezüglich des hydraulischen Potentials des Bodenwassers unterscheidet es sich durch die Arbeit, die gegen die Schwerkraft und gegen die Widerstände der Wurzeln und der Leitgefäße in den Bäumen beim Transport in die Nadeln verrichtet werden muß. Das Nadelwasserpotential $\Psi_{w, k}$ in der Schicht $k$ wird bestimmt durch das hydraulische Potential des Wassers in den verschiedenen wurzeldurchsetzten Bodenschichten (das mittlere Wurzelwasserpotential $\Psi_{\text {root }}$ ), durch die Transpirationsrate $E_{t r, k}\left[\mathrm{kgm}^{-2} \mathrm{~s}^{-1}\right]$ und durch die Widerstände bei der Wasseraufnahme durch die Wurzeln (mittlerer Wurzelwiderstand $\overline{r_{\text {root }}}[\mathrm{s}]$ - mittlerer Widerstand gegen den Wassertransport vom Boden in die Wurzeln) und beim Wassertransport durch Stamm, Äste und Zweige (mittlerer Pflanzenwiderstand $\overline{r_{p l a n t}}[\mathrm{~s}]$ ). Nach van der Honert 1948, genannt in [35] wird das Nadelwasserpotential berechnet nach:

$$
\Psi_{w, k}=\Psi_{\text {root }}-z_{k}-E_{t r, k} \frac{\overline{r_{\text {plant }}}+\overline{r_{\text {root }}}}{\rho_{w}} .
$$

Das mittlere Wurzelwasserpotential $\Psi_{\text {root }}$ ergibt sich als gewichtetes arithmetisches Mittel der Bodenwasserpotentiale $\Psi_{i}$ aller Bodenschichten aus denen die Wurzeln Wasser extrahieren (Wurzelzone). Als Wichtungsfaktoren treten die Dicken der Bodenschichten $h_{b, i}$ auf [35]:

$$
\Psi_{\text {root }}=\frac{1}{z_{\text {root }}} \sum_{0}^{z_{\text {root }}} \Psi_{i} h_{b, i} .
$$

$z_{\text {root }}$ ist die durch Messungen bestimmte maximale Wurzeltiefe. Der Widerstand $\overline{r_{\text {plant }}}$ wird als zeitlich konstant angenommen mit einem Wert von $\overline{r_{\text {plant }}}=$ $50 \mathrm{~s}$. Der mittlere Wurzelwiderstand $\overline{r_{\text {root }}}$ wird berechnet nach der Formel von Federer 1979, genannt in [35]:

$$
\begin{gathered}
\overline{r_{\text {root }}}=\frac{R / D_{d}+\alpha_{f} / K_{\text {root }}}{z_{\text {root }}} \\
\alpha_{f}=\frac{1}{8 \pi D_{d}}\left[V_{r}-3-2 \ln \left(\frac{V_{r}}{1-V_{r}}\right)\right],
\end{gathered}
$$




$$
\begin{array}{lll}
\text { mit: } & & \\
R & - & \text { Transportwiderstand pro Meter Wurzellänge }\left[\mathrm{ms}^{-1}\right] \\
D_{d} & - & \text { Wurzeldichte }=\text { Wurzellänge pro Kubikmeter Boden }\left[\mathrm{m} \mathrm{m}^{-3}\right] \\
V_{r} & - & \text { Volumen an Wurzelmasse pro Volumen Boden }\left[\mathrm{m}^{3} \mathrm{~m}^{-3}\right] \\
K_{\text {root }} & & \text { mittlere hydraulische Leitfähigkeit in der Wurzelzone }\left[\mathrm{m} \mathrm{s}^{-1}\right] .
\end{array}
$$

Für die Parameter $R, D_{d}$ und $V_{r}$ werden gemessene Werte verwendet [12]. $K_{r}$ berechnet sich aus dem mittleren Wurzelwasserpotential $\Psi_{r}$ nach [35]:

$$
K_{\text {root }}=\overline{K_{s}}\left(\frac{\overline{\Psi_{s}}}{\Psi_{\text {root }}}\right)^{\frac{(2 \bar{B}+3)}{\bar{B}}} .
$$

Dabei ist $\overline{K_{s}}$ die mittlere hydraulische Leitfähigkeit des Bodens in der Wurzelzone bei Wassersättigung, $\bar{\Psi}_{s}$ das mittlere hydraulische Potential des Bodenwassers in der Wurzelzone bei Wassersättigung und $\bar{B}$ der mittlere Wert des empirischen Parameters $B$ in der Wurzelzone. Im Modell werden die Werte von $\overline{K_{s}}, \overline{\Psi_{s}}$ und $\bar{B}$ in Analogie zur Bestimmung von $\Psi_{\text {root }}$ über ein mit den Bodenschichtdicken gewichtetes arithmetisches Mittel der jeweiligen Parameter jeder Bodenschicht bestimmt. Die Transpirationsrate der Nadeln einer Bestandsschicht $k$ ergibt sich als Teil der Evapotranspiration nach Gl. 5.32, indem nur der nicht vom Niederschlag benetzte Teil der Nadeloberfläche betrachtet wird, zu [35]:

$$
L E_{t r, k}=\frac{\rho c_{p}}{\gamma_{k}} m\left(e^{*}\left(T_{w, k}\right)-e_{k}\right) \frac{1-N_{k}}{r_{b, k}+r_{s, k}} .
$$

Nach der Berechnung des Nadelwasserpotentials aller Bestandsschichten mit Kenntnis der Transpirationsraten kann die Wasserentnahme (Extraktion) $E_{x t}$ der Wurzeln in jeder Bodenschicht $\mathrm{k}$ aufgrund der Transpiration berechnet werden. Dabei wird im Modell eine abgewandelte Formel nach [35] angewendet. Im Simple Biosphere Model von Sellers et al. (1986) wird die Gesamttranspiration der Vegetationsschicht betrachtet, nicht aber die vertikale Verteilung der Transpiration im Bestand, wie wir sie zur Berechnung der Energieflüsse Vegetation - Luft in den Modellschichten benötigen. Aus diesem Grunde wurde bei Sellers et al. auch nur ein Nadelwasserpotential für die gesamte Vegetationsschicht verwendet, während im vorliegenden Modell das Nadelwasserpotential vertikal aufgelöst wird. Dies ist die Ursache dafür, weshalb in der folgenden Beziehung das Nadelwasserpotential für den gesamten Bestand wie bei Sellers et al. durch das mittlere Nadelwasserpotential aller mit Nadeln besetzten Bestandesschichten $\overline{\Psi_{v}}$ und die effektive Höhe der Transpirationsquelle durch die mittlere Höhe des Kronenraumes $\overline{h_{v}}$ ersetzt wurde:

$$
E_{x t, k}=\frac{h_{b}(k)}{z_{\text {root }}}\left(\frac{\Psi_{k}-\overline{\Psi_{v}}-\overline{h_{v}}}{\overline{r_{\text {plant }}}-\overline{r_{\text {soil }}}}\right) \rho_{w} .
$$

Sollte sich nach dieser Formel ein negativer Wert für $E_{x t, k}$ ergeben so wird $E_{x t, k}$ auf Null gesetzt, da eine negative Extraktionsrate einen Rückfluß von Wasser aus den Wurzeln bedeuten würde. In Waldbeständen ist dieser Prozeß allein aufgrund des Wassergehaltsgradienten Wurzel - Boden extrem selten (extreme Dürre) und würde eine Zerstörung des Wurzelwerkes zur Folge haben.

In den genannten Beziehungen müssen das Nadelwasserpotential und das hydraulische Potential des Bodenwassers in der Einheit [m] eingesetzt werden. 


\begin{tabular}{|c|c|c|c|}
\hline Bezeichnung & Beschreibung & Wert & Quelle \\
\hline$\alpha_{g}$ & kurzwellige Albedo der Bodenoberfläche & 0.1 & [45] \\
\hline$\alpha_{w}$ & kurzwellige Albedo der Vegetat.-oberfl. & 0.1 & $"$ " \\
\hline$\epsilon_{g}$ & $\begin{array}{l}\text { langwelliger Emissionskoeffizient } \\
\text { der Bodenoberfläche }\end{array}$ & 0.93 & “ \\
\hline$\epsilon_{w}$ & $\begin{array}{l}\text { langwelliger Emissionskoeffizient } \\
\text { der Baumoberfläche }\end{array}$ & 0.98 & “ \\
\hline$r_{s, \min }$ & minimaler Stomatawiderstand & $150 \mathrm{sm}^{-1}$ & [29] \\
\hline & $\begin{array}{l}\text { Param. für den Einfl. der PAR-Strahl. } \\
\text { auf den Stomatawiderstand }\end{array}$ & $6.35 \cdot 10^{-7}$ & " \\
\hline$T_{\min }$ & $\begin{array}{l}\text { untere Grenztemperatur für Öffnung } \\
\text { der Stomata }\end{array}$ & $273.15 \mathrm{~K}$ & “ \\
\hline$T_{\text {opt }}$ & $\begin{array}{l}\text { optimale Temperatur für minimalen } \\
\text { Stomatawiderstand }\end{array}$ & $299.86 \mathrm{~K}$ & “ \\
\hline$T_{\max }$ & $\begin{array}{l}\text { obere Grenztemperatur für Öffnung } \\
\text { der Stomata }\end{array}$ & $310.59 \mathrm{~K}$ & “ \\
\hline$\alpha$ & Parameter für Abhängigkeit des & $6.35 \cdot 10^{-2} \mathrm{hPa}^{-1}$ & “ \\
\hline$\Psi_{1}$ & $\begin{array}{l}\text { Stomatawiderst. vom Sättigungsdefizit } \\
\text { obere Grenze des Blattwasserpot. } \\
\text { für offene Stomata }\end{array}$ & $-10^{4} \mathrm{hPa}$ & " \\
\hline$\Psi_{2}$ & $\begin{array}{l}\text { untere Grenze des Blattwasserpot. } \\
\text { für offene Stomata }\end{array}$ & $-2.5 \cdot 10^{4} \mathrm{hPa}$ & " \\
\hline$z_{\text {root }}$ & maximale Wurzeltiefe & $1 \mathrm{~m}$ & [12] \\
\hline$D_{d}$ & Wurzeldichte & $783 \mathrm{~mm}^{-3}$ & $"$ \\
\hline$R$ & Transportwiderst. pro $\mathrm{m}$ Wurzellänge & $5 \mathrm{~ms}^{-1}$ & " \\
\hline$V_{r}$ & Volumenanteil des Wurzelwerks & $0.02 \mathrm{~m}^{3} \mathrm{~m}^{-3}$ & “ \\
\hline$\overline{r_{\text {plant }}}$ & $\begin{array}{l}\text { Transportwiderstand in Stamm, } \\
\text { Ästen und Zweigen }\end{array}$ & $50 \mathrm{~s}$ & [29] \\
\hline
\end{tabular}

Tabelle 5.5: Zur Modellierung der Energie- und Feuchteflüsse zwischen Vegetation bzw. Boden und Atmosphäre benutzte Parameter.

\subsection{Behandlung der Niederschläge}

Aufgrund der Interzeption von Niederschlagswasser und der Beeinflussung der Bodenevaporation haben Niederschläge einen nicht zu vernachlässigenden Einfluß auf den Stoff- und Energieaustausch zwischen der Vegetation bzw. dem Erdboden und der Atmosphäre. So fällt bis zu etwa 40 \% (Abschätzung nach Brechtel (1987), genannt in [17]) der über dem betrachteten Fichtenbestand gemessenen Niederschlagsmenge (= Freiflächenniederschlag) der Interzeptionsverdunstung "zum Opfer" und gelangt somit nicht zum Waldboden. Ibrom et al. selbst geben in der genannten Arbeit einen Wert von $25 \%$ des Freiflächenniederschlags an. Das steht jedoch nicht im Widerspruch zum letztgenannten Wert, da die absolute Menge als auch der relative Anteil der Interzeptionsverdunstung pro Jahr mit der Jahresniederschlagsmenge stark schwankt [17]. Beim Fallen des Niederschlages durch die Bestandsschicht wird also die über dem Bestand zu messende Niederschlagsrate aufgespalten in den Anteil, der an der Baumoberfläche haften bleibt (Interzeptionsrate), und den Anteil, der weiter zu Boden fällt (effektive Niederschlagsrate). Die effektive Niederschlagsrate $P_{\text {eff. }}\left[\mathrm{ms}^{-1}\right]$ unterliegt dabei einem ähnlichen Gesetz, wie es zur Beschreibung der Absorption der kurzwelligen Sonnenstrahlung im Bestand verwendet wird (exponentielle Abnahme mit zunehmendem Eindringen in 
den Bestand, vgl. Gl. 5.19) [35]:

$$
P_{e f f, k}=n_{w} P_{h} \exp \left(-k_{0} L_{v}\left(z_{k}\right)\right) \text {. }
$$

$P_{h}$ ist die Niederschlagsrate in Höhe der Bestandsobergrenze, $n_{w}$ ist der Anteil der horizontalen Gesamtfläche, die im Mittel vom Baumquerschnitt eingenommen wird. Der Parameter $k_{0}$ ist der "Extinktions" -Koeffizient für den Niederschlag - er ist gleich dem Extinktionskoeffizienten für direkte kurzwellige Strahlung für eine Baumoberfläche, die einem Schwarzen Körper entspricht [35], da die Reflexion beim Niederschlag entfällt.

Die Interzeptionsrate ergibt sich aus der Niederschlagsrate in Höhe der Bestandsobergrenze, bezogen auf den Anteil $n_{w}$, indem die effektive Niederschlagsrate subtrahiert wird:

$$
P_{\text {int }, k}=n_{w} P_{h}\left[1-\exp \left(-k_{0} L_{v}\left(z_{k}\right)\right)\right] .
$$

Das interzeptierte Niederschlagswasser $M_{i n t, k}[\mathrm{~m}]$ bleibt zum Teil an der Baumoberfläche haften, kann aber auch an der Baumoberfläche weiter nach unten abfließen oder abtropfen (Drainage), wenn die maximal mögliche Menge an Wasser, die von der entsprechenden Schicht im Bestand gehalten werden kann, $M_{\max }$, überschritten wird. Damit ergibt sich für die Drainagerate $P_{d r, k}$ in der Schicht $k$ die folgende Beziehung [35]:

$$
P_{d r, k}= \begin{cases}0 & \text { für } \mathrm{M}_{i n t, k}<M_{\text {max }, k} \\ P_{i n t, k} & \text { für } \mathrm{M}_{i n t, k}=M_{\text {max }, k} .\end{cases}
$$

Für $M_{\text {max }}$ gibt es in der Literatur unterschiedliche Angaben. Bei diesem Parameter spielen die Art des Bestandes und die Größe der Blattoberfläche eines Baumes eine Rolle. Bei Sellers et al. [35] wird ein Bereich von $M_{\max , k} \approx(2$. $\left.10^{-4} \mathrm{~m}^{2}-5 \cdot 10^{-4} \mathrm{~m}^{2}\right) \cdot A_{\text {tree }, \mathrm{k}}$ als Abhängigkeit von der Baumoberflächendichte angegeben, so daß die Verwendung eines Wertes $M_{\max , k}=4 \cdot 10^{-4} \mathrm{~m}^{2} \cdot \mathrm{A}_{\text {tree, } \mathrm{k}}$ im vorliegenden Modell berechtigt erscheint. Der Sinn der Berechnung der Drainagerate liegt darin, daß diese für die Berechnung des effektiven Niederschlages $P_{0}$ an der Bodenoberfläche berücksichtigt werden muß. Für $P_{0}$ ergibt sich somit [35]:

$$
P_{0}=P_{h}-\sum_{z_{k}=0}^{h_{p}} P_{i n t, k}+\sum_{z_{k}=0}^{h_{p}} P_{d r, k} .
$$

Mit Hilfe der Menge an Interzeptionswasser pro Bestandsschicht kann der Anteil bestimmt werden, den dieses auf der Oberfläche der Vegetation der Bestandsschicht einnimmt. Dieser Wert ist für die Berechnung der Transpirationsund Evaporationsraten wichtig. Nach Sellers et al. [35] wird das Verhältnis der Massen- bzw. Volumina dem Verhältnis der Oberflächen gleichgesetzt. Somit ergibt sich für den mit Interzeptionswasser benetzten feuchten Anteil $N_{k}$ der gesamten Baumoberfläche einer Schicht $k$ :

$$
N_{k}= \begin{cases}\frac{M_{i n t, k}}{M_{m a x, k}} & \text { für } \mathrm{e}^{*}\left(T_{w, k}\right)>e\left(T_{k}\right) \\ 1 & \text { für } \mathrm{e}^{*}\left(T_{w, k}\right)<e\left(T_{k}\right),\end{cases}
$$

wobei $e^{*}\left(T_{w, k}\right)$ der Sättigungsdampfdruck an der Baumoberfläche und $e\left(T_{k}\right)$ der Dampfdruck in der umgebenden Luft ist. 


\begin{tabular}{lll}
\hline \hline Bezeichnung & Beschreibung & Wert \\
\hline$R_{c}$ & nichtlokale kritische Richardsonzahl & 0.5 \\
$D$ & Dissipationsparameter & $5 \cdot 10^{-6} \mathrm{~s}^{-2}$ \\
$\beta$ & $\begin{array}{l}\text { Normierungsparameter bei der } \\
\text { Berechnung der Transilienzmatrix aus } \\
\text { dem Mischungspotential }\end{array}$ & $5 \cdot 10^{-4}$ \\
$Y_{\text {ref }}$ & $\begin{array}{l}\text { Parameter für den subgridskaligen } \\
\text { turbulenten Austausch }\end{array}$ & 0.5 \\
\hline \hline
\end{tabular}

Tabelle 5.6: Im Modell verwendete Parameter der transilienten Turbulenzschließung. Quelle: [18]

\subsection{Festlegung der Parameter der transilienten Schlies- sung}

Das Mischungspotential wird bei den Modellrechnungen nach Gleichung 3.21 parametrisiert. Zusammen mit dem Übergang zur Transilienzmatrix benötigt der transiliente Schließungsansatz 4 grundlegende Parameter, denen nicht in jedem Falle eine erschöpfende physikalische Bedeutung zugewiesen werden kann, so daß deren Werte auch unter Zuhilfenahme von Testsimulationen und Vergleich der Ergebnisse mit vorliegenden Meßwerten gewonnen werden müssen. Es handelt sich dabei um die nichtlokale kritische Richardsonzahl $R_{c}$, einen nichtlokalen Dissipationsparameter $D$, der die Dissipation der nichtlokal definierten Turbulenzenergie in Wärmeenergie beschreibt, einen Parameter $\beta$, der die Reaktion der Transilienzmatrix bei der Umrechnung auf verschiedenen Zeitschritte beschreibt und einen Parameter $Y_{\text {ref } f}$ zur Beschreibung der schichtinternen (subskaligen) Turbulenz. Die Einführung dieser Parameter wurde in Kapitel 3 beschrieben.

Die Werte dieser 4 Parameter sind in Tabelle 5.6 dargestellt. Für die hier vorliegenden Berechnungen wurden die Parameterwerte von [18] übernommen, da sie sich bei den Rechnungen dieser Arbeitsgruppe schon als brauchbar erwiesen haben. 


\section{Kapitel 6}

\section{Validierung des Modells und Vergleichsrechnungen}

Bevor ein Modell für vielfältige Berechnungen eingesetzt wird, muß es auf seine Brauchbarkeit überprüft werden. Das soll in diesem Kapitel geschehen. In unserem Falle stellen sich insbesondere die folgenden Fragen:

- Kann das Grenzschichtmodell mit transilienter Turbulenzschließung die wesentlichen Prozesse in einem Waldbestand qualitativ oder auch quantitativ richtig widergeben?

- Reichen stationäre Rechnungen, um den turbulenten Austausch in der Atmosphäre zu quantifizieren, oder sind instationäre Rechnungen notwendig ?

- Liefern die Rechnungen mit transilienter Turbulenzschließung bessere Ergebnisse als bei Verwendung des K-Schließungsansatzes?

Diese und andere Fragen sollen in diesem Kapitel beantwortet werden.

\subsection{Auswahl des zu modellierenden Zeitraumes}

Aufgrund der vielfältigen Eingangsgrößen des Modells, muß bei der Auswahl des zu modellierenden Zeitraumes auf das möglichst vollständige Vorhandensein der Modelleingangsparaneter geachtet werden. Im Falle des hier vorliegenden atmosphärischen Grenzschichtmodells heißt dies vor allem, daß Meßwerte der atmosphärischen Eingangsgrößen (Temperatur, Windgeschwindigkeit, spezifische Feuchte) im Bestand möglichst vollständig vorhanden sein müssen. Einzelne Ausfallwerte können dabei durch Interpolation eliminiert werden. Fehlt das Profil einer Eingangsgröße völlig, so müssen insbesondere Annahmen über deren Vertikalprofil im Bestand getroffen werden. Hier kann zum Beispiel ein mittleres Profil dieser Größe im Bestand für den entsprechenden Monat oder den entsprechenden Startzeitpunkt helfen. Im allgemeinen bleibt jedoch das Vorhandensein von Meßwerten aller Eingangs- 
größen und den Analysewerten des Wetterdienstmodells über dem Bestand wünschenswert. Neben den obengenannten Bedingungen sollte das Modell auch an Zeiträumen getestet werden, an denen die Stärken und Schwächen des Modells deutlich zutage treten können.

Für die folgenden Untersuchungen zur Leistungsfähigkeit des transilienten Schließungsansatzes wurden der 20. Juni und der 21. Juni 1998 ausgewählt. An diesen beiden Tagen herrschte sommerliches Hochdruckwetter im betrachteten Gebiet, mit gut ausgebildeter Konvektion (am 22. Juni löste die Konvektion dann auch verbreitet Gewitter aus) bei geringen Windgeschwindigkeiten. Da man bei der Definition des transilienten Schließungsansatzes die Turbulenz als Prozeß advektiver vertikaler Transporte betrachtet, ist der ausgewählte Zeitraum gut geeignet, um die Möglichkeiten des transilienten Schließungsansatzes zu untersuchen.

\subsubsection{Parametrisierung der Nachlaufturbulenz}

Für die Berücksichtigung der Nachlaufturbulenz wurde ein relativ einfacher Ansatz nach [18] verwendet. Um die Auswirkungen diese Termes auf die Modellergebnisse festzustellen wurden instationäre Rechnungen mit und ohne Berücksichtigung dieses Termes für den 20. Juni 1998 durchgeführt. Die Ergebnisse dieser Rechnungen zeigten jedoch in den Profilen und in den Eigenschaftsflüssen keine erkennbaren Unterschiede. Bei den weiteren Rechnungen wurde deshalb der Term zur Berücksichtigung der Nachlaufturbulenz bei der Berechnung des Mischungspotentials vernachlässigt.

\subsection{Strahlungsmodellierung}

Um die Modellergebnisse besser beurteilen zu können, ist es wichtig die Güte der Parametrisierungen der einzelnen Modellteile zu kennen. Dazu soll zunächst der Strahlungsteil des Modells betrachtet werden, der den Transport der lang- und kurzwelligen Strahlung im Bestand parametrisiert.

Die Abb. 6.2 zeigt den modellierten kurzwelligen abwärtsgerichteten Strahlungsfluß (Globalstrahlung) für den 20. und 21. Juni 1998 in 2m Höhe im Vergleich zum gemessenen kurzwelligen Strahlungsfluß. Was sofort ins Auge fällt, sind die teilweise größeren Unterschiede zwischen beiden Kurven. Bei allen Parametrisierungen, die (wenn auch manchmal nur zum Teil) auf empirischen gewonnenen Größen beruhen, kann das eindimensionale Modell nur den mittleren Zustand des Bestandes wiedergeben. Lokale Besonderheiten (z.B. eines Baumes) können nicht berücksichtigt werden. Das ist insbesondere bei der Strahlungsmodellierung der Fall. Wie man aus der Abb. ersehen kann, liegen die berechneten Werte zwar größenordnungsmäßig im Bereich der gemessenen Werte, aber es gibt dennoch größere Abweichungen. So werden in den Morgen- und in den Abendstunden zu niedrige Werte modelliert, was einmal darauf hindeuten kann, daß die Sonnenstrahlung zu diesen Zeiten durch Lücken im Kronendach häufiger direkt in den Stammraum und auf die Bodenoberfläche scheint, was im Modell keine Berücksichtigung findet. Zum anderen wird die diffuse kurzwellige Strahlung mitgemessen. Diese Strahlung 


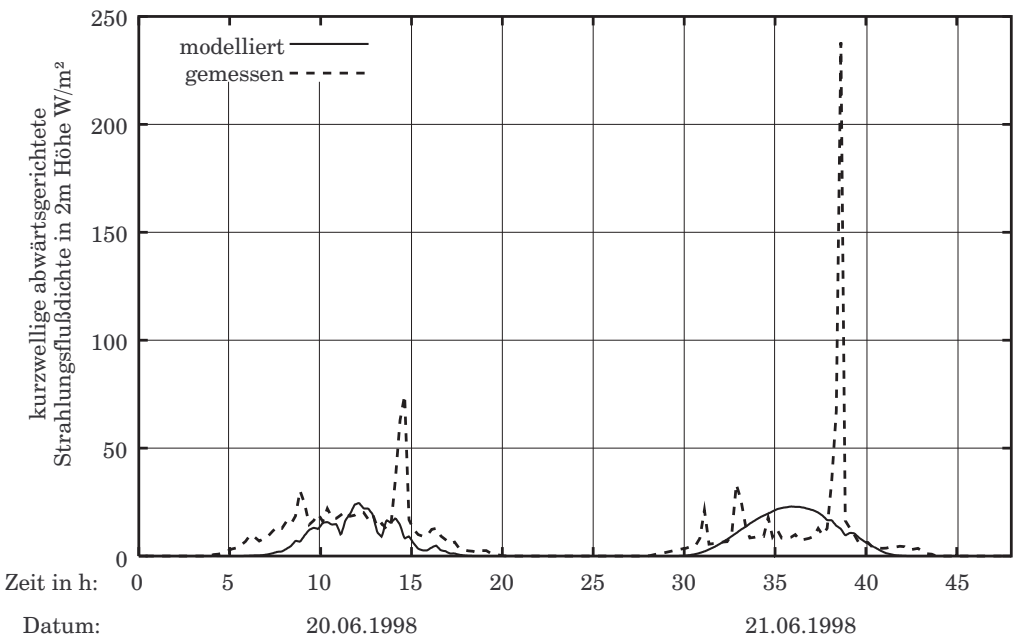

Abbildung 6.1: Vergleich der gemessenen und berechneten kurzwelligen abwärtsgerichteten Strahlungsflußdichte in 2m Höhe für den 20. und 21.06.1998 nach Meßdaten und Modellberechnungen

verhält sich in vielerlei Beziehung anders als die direkte Strahlung und wird in den Strahlungstermen nicht explizit berücksichtigt. Auffällig ist desweiteren, daß an beiden Tagen um etwa 14:00 Uhr herum sehr hohe Strahlungswerte gemessen werden, was nur dadurch erklärt werden kann, daß der Strahlungssensor zu dieser Zeit fast direkt der Sonnenstrahlung ausgesetzt war sich also im Bereich eines ",Sonnenflecks" befand. An diesen beiden Stellen mit extrem hohen Meßwerten ist zu Vermuten, daß der Antrieb der Strahlungsschiene (siehe Abschnitt 5.2) zeitweise ausgefallen ist und dadurch nicht immer eine horizontale Mittelung der Strahlungswerte möglich war.

\subsection{Bodentemperaturmodellierung}

Die Abb. 6.3 zeigt einen Vergleich der Profile der Erdbodentemperaturen, wie sie das Modell für den 20. Juni 1998 simuliert hat und wie sie während dieses Tages gemessen wurden. Die Rechnung wurde um 0:30 Uhr gestartet. Es ist eine prinzipielle Übereinstimmung zwischen Simulation und Messung festzustellen, was auch kaum anders überrascht, handelt es sich bei der Wärmeleitungsgleichung doch um einen relativ einfachen Fall einer partiellen Differentialgleichung. Unterschiede lassen sich durch die in der Simulation nicht berücksichtigte Tatsache erklären, daß durch die im Boden stattfindenden Wassertransporte auch Wärme mittransportiert wird. Prinzipiell kann man also feststellen, daß das Bodenmodell in der Lage ist, den tageszeitlichen Verlauf der Bodentemperaturen richtig widerzugeben. 

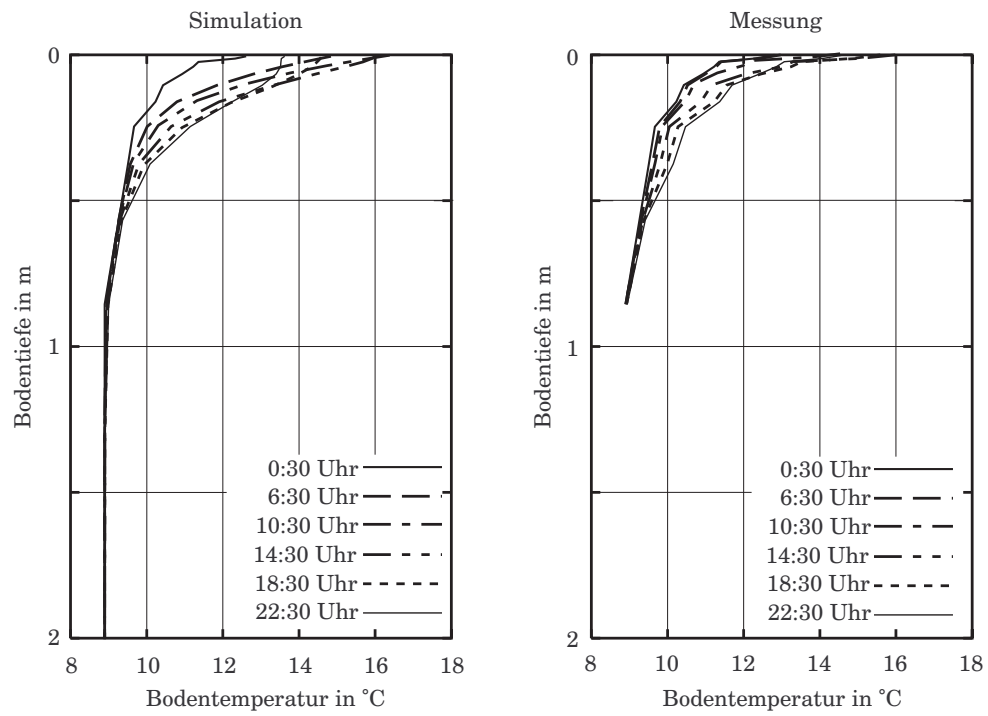

Abbildung 6.2: Berechnete und gemessene Bodentemperaturen für den 20.06.1998

\subsection{Bodenfeuchtemodellierung}

Die Abb. 6.4 zeigt den simulierten zeitlichen Verlauf der Profile der relativen Bodenfeuchte für den 20. Juni 1998. Die Rechnung wurde um 0:30 gestartet. Die relative Bodenfeuchte ist das Verhältnis der aktuellen volumetrischen Bodenfeuchte $\left(\mathrm{m}^{3}\right.$ Wasser pro $\mathrm{m}^{3}$ Boden) zur maximal möglichen volumetrischen Bodenfeuchte, bei deren Überschreiten Versickerung durch Gravitation auftritt. Leider gibt es für die Bodenfeuchte keine kontinuierlichen Messungen, wie dies für die Bodentemperatur der Fall ist. Aus diesem Grunde können die berechneten Profile in diesem Falle nicht mit Meßwerten verglichen werden. Prinzipiell richtig erscheint aber die allgemeine Zunahme der Bodenfeuchte mit der Bodentiefe als Folge der Quellen- bzw. Senkenverteilung (Grundwasserspiegel bzw. Erdoberfläche und Wurzeln). Auch nimmt in den oberen Bodenschichten der Wassergehalt im Laufe des Tages als Folge der Wasseraufnahme durch die Wurzeln und der direkten Evaporation an der Erdoberfläche kontinuierlich ab. Allerdings wurde der starke Gradient der Bodenfeuchte zwischen $50 \mathrm{~cm}$ und $1 \mathrm{~m}$ Tiefe von anderen Autoren (z. B. in [18]) nicht beobachtet.

\subsection{Stationäre Modellrechnungen}

Da die Transilienzmatrix zur Beschreibung des vertikalen turbulenten Austausches nur von den Profilen der horizontalen Windgeschwindigkeit, der Lufttemperatur und der spezifischen Feuchte (die spezifische Feuchte wird zur Berechnung der virtuellen potentiellen Temperatur benötigt) abhängt, stellt sich die Frage, ob aus vorliegenden Profilen dieser Größen auf direktem Wege, also 


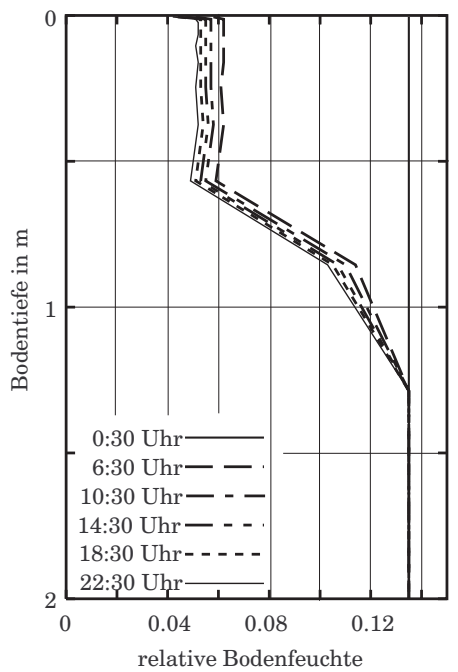

Abbildung 6.3: Berechnete Profile der relativen Bodenfeuchte für den 20.06.1998

mittels stationärer Rechnung, die Transilienzmatrix und somit die vertikalen turbulenten Flüsse berechnet werden können.

Man müßte also eigentlich nur das Mischungspotential aus den vorgegebenen Profilen berechnen, die Transilienzmatrix bestimmen und daraus dann die turbulenten Flüsse. Diesem Ansatz steht das sogenannte „split - timestep“ Verfahren im Wege (siehe Abschnitt 3.8), da erst die Eigenschaftsquellen und senken berechnet werden müssen und mit deren Hilfe eine "Destabilisierung" der Profile erfolgen muß, ehe eine brauchbare Transilienzmatrix und somit die turbulenten Flüsse berechnet werden können. Dieses Problem wurde dadurch gelöst, indem alle Modellgleichungen einschließlich der transilienten Schließungsparametrisierung zu einem festen Zeitpunkt und bei festgehaltenen Randbedingungen solange integriert werden, bis ein stationärer Zustand erreicht ist.

Es wurden im Bestand Meßwerte und über dem Bestand die Analysewerte des DWD-Deutschlandmodells verwendet. Während jeder Rechnung wurden die gemessenen Werte der Eigenschaften im Bestand festgehalten, da die turbulenten Flüsse ja für diese Eigenschaftsprofile berechnet werden sollen. Desgleichen wurden auch die Analysewerte des DWD-Modells oberhalb $2000 \mathrm{~m}$ festgehalten, um einerseits den Charakter der atmosphärischen Turbulenz über der Grenzschicht zu erhalten, andererseits aber die Ausbildung der atmosphärischen Grenzschicht in den unteren $2000 \mathrm{~m}$ durch das transiliente Modell zu ermöglichen.

Als Kriterium für das Erreichen eines stationären Zustandes wird das Profil der Mischungsweglänge verwendet. Wenn sich die Summe der für alle Modellschichten berechneten Mischungsweglängen zwischen zwei Rechenschritten nicht mehr als um $1 \%$ ändert, wurde die stationäre Rechnung abgebrochen und die berechnete Transilienzmatrix und die berechneten Profile als Ergebnis 
der stationären Rechnung verwendet.

Der Gebrauch der Mischungsweglänge als Kriterium für das Erreichen des stationären Zustandes hat den Vorteil, daß das Profil der Mischungsweglänge von allen Größen abhängt, die auch in die Berechnung der Transilienzmatrix eingehen. So könnte es bei Verwendung der horizontalen Windgeschwindigkeit als Kriterium geschehen, daß zwar ein stationäres Profil der Windgeschwindigkeit erreicht wird, das Profil der Lufttemperatur aber noch größeren Änderungen unterworfen ist, und dadurch irrtümlicherweise das Erreichen eines stationären Zustandes angezeigt wird.

Die Abbildung 6.5 zeigt die mittels transilientem Modell in stationärer Rechnung modellierten turbul. Flüsse für Impuls, fühlbare und latente Wärme zu unterschiedlichen Zeiten für den 20.06.1998.

Für die Beurteilung der berechneten Eigenschaftsflüsse standen keine gemessenen Profile der Flüsse innerhalb des Bestandes, sondern nur Flußmessungen in einer Höhe (39 m) über dem Bestand zur Verfügung, die mit Hilfe der Eddy-Kovarianzmethode bestimmt wurden. An die Diskussion der berechneten Flussprofile schließt sich deshalb ein Vergleich der berechneten und gemessenen Flüsse in $39 \mathrm{~m}$ Höhe an.

Die berechneten Impulsflüsse innerhalb des Bestandes sind in allen Höhen abwärtsgerichtet und weisen einen deutlichen Tagesgang mit niedrigeren Werten nachts und höheren Werten am Tage auf. Insoweit wird die Bedeutung der Erdoberfläche und des Bestandes, sowie die tageszeitliche Variation der Intensität des turbulenten Austausches zum Ausdruck gebracht. Im Stammraum und im unteren Kronenraum bis etwa 20 m Höhe bleiben die berechneten Flüsse relativ klein. Das kann mit den geringeren Vertikalgradienten der Windgeschwindigkeiten in diesem Bereich, aber auch mit der Abschwächung des vertikalen Austausches mit den höheren Luftschichten durch das Kronendach begründet werden. Zusätzlich wurde durch das gemessene Temperaturmaximum im Kronenraum während des Tages eine stabile thermische Schichtung im unteren Kronenraum festgestellt, die den Austausch in diesem Bereich ebenfalls erschwert. Die höchsten Absolutbeträge der Impulsflüsse werden zu allen Tageszeiten direkt in Höhe der Bestandesobergrenze modelliert. Wie auch bei den anderen Eigenschaftsflüssen kommt die charakteristische Höhenkonstanz der turbulenten Flüsse in der direkt über dem Bestand liegenden PrandtlSchicht nicht deutlich zum Ausdruck, da die Absolutbeträge der Flüsse nach dem Erreichen ihres Maximums mit zunehmender Höhe wieder zurückgehen.

Wie schon der Impulsfluß, zeigt auch der fühlbare Wärmefluß einen deutlichen Tagesgang, der der tageszeitlich unterschiedlich starken Erwärmung der Luft im Kronenraum zugeschrieben werden kann. Dabei werden die höchsten Werte an der Bestandesobergrenze mit etwa $230 \mathrm{~W} / \mathrm{m}^{2}$ schon um 10:30 Uhr prognostiziert. Bis auf die Ausnahme des Profiles um 18:30 Uhr ist der turbulente Transport immer aus dem Bestand heraus gerichtet. Der Bestand wirkt offensichtlich im Modell als Wärmequelle für die Luft der gesamten Modellatmosphäre. Die im Modell mit eingeschlossene Wärmespeicherung in der Biomasse reicht dabei offensichtlich aus, auch nachts einen über dem Bestand nach oben gerichteten fühlbaren Wärmefluss zu generieren.

Der latente Wärmefluß innerhalb des Bestandes zeigt einen ähnlich ausge- 

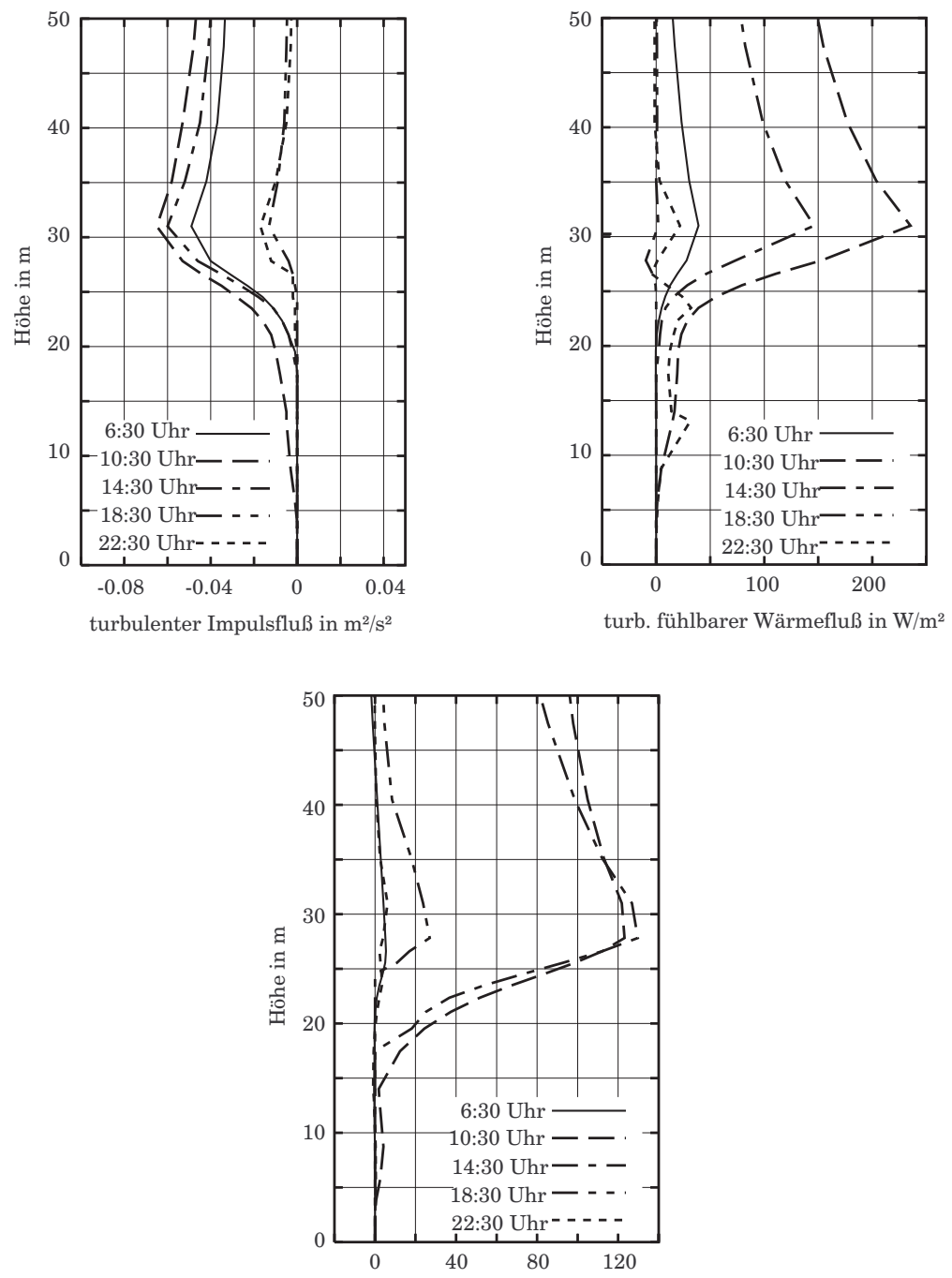

turbul. latenter Wärmefluß in W/m²

Abbildung 6.4: Berechnete turbul. Impulsflüsse und fühlbare Wärmeflüsse (oben) sowielatente Wärmeflüsse (unten) in und über dem Bestand für den 20.06 .1998 bei stationärer Rechnung mit dem transilienten Modell 
prägten Tagesgang, wie der fühlbare Wärmefluß. Die erreichten Maximalwerte an der Obergrenze des Bestandes fallen allerdings mit z.B. $130 \mathrm{~W} / \mathrm{m}^{2}$ um 10:30 Uhr deutlich geringer aus.

\begin{tabular}{|c|c|c|c|}
\hline \multicolumn{4}{|c|}{ Impulsfluß in $\mathrm{m}^{2} / \mathrm{s}^{2}$ : } \\
\hline Uhrzeit & berechnet & gemessen & $\begin{array}{l}\text { Anteil am } \\
\text { gemessenen Abso- } \\
\text { lutbetrag }\end{array}$ \\
\hline 6:30 & -0.04 & -0.11 & $63 \%$ \\
\hline 10:30 & -0.05 & -0.17 & $70 \%$ \\
\hline 14:30 & -0.04 & -0.26 & $85 \%$ \\
\hline 18:30 & -0.01 & -0.01 & $100 \%$ \\
\hline 22:30 & -0.01 & -0.06 & $83 \%$ \\
\hline \multicolumn{4}{|c|}{$\begin{array}{ll} & \text { fühlbarer Wärmefluß in } \mathrm{W} / \mathrm{m}^{2} \text { : }\end{array}$} \\
\hline Uhrzeit & berechnet & gemessen & $\begin{array}{l}\text { Anteil am } \\
\text { gemessenen Abso- } \\
\text { lutbetrag }\end{array}$ \\
\hline $6: 30$ & 23.6 & 14.7 & $161 \%$ \\
\hline 10:30 & 176.8 & 165.1 & $107 \%$ \\
\hline 14:30 & 98.6 & 198.4 & $50 \%$ \\
\hline 18:30 & 0.9 & 18.9 & $5 \%$ \\
\hline 22:30 & -1.4 & -24.2 & $6 \%$ \\
\hline \multicolumn{4}{|c|}{$\begin{array}{ll} & \text { latenter Wärmefluß in } \mathrm{W} / \mathrm{m}^{2} \text { : }\end{array}$} \\
\hline Uhrzeit & berechnet & gemessen & $\begin{array}{l}\text { Anteil am } \\
\text { gemessenen Abso- } \\
\text { lutbetrag }\end{array}$ \\
\hline $6: 30$ & 1.1 & 6.1 & $18 \%$ \\
\hline 10:30 & 104.9 & 194.6 & $54 \%$ \\
\hline 14:30 & 98.6 & 186.9 & $53 \%$ \\
\hline 18:30 & 8.4 & 30.2 & $28 \%$ \\
\hline 22:30 & 0.6 & 0.0 & - \\
\hline
\end{tabular}

Tabelle 6.1: Vergleich der mit dem transilienten Modell in stationärer Rechnung berechneten turbulenten Flüsse von Impuls, fühlbarer und latenter Wärme in $39 \mathrm{~m}$ Höhe am 20.06.1998 mit den mittels Eddy - Kovarianz Verfahren bestimmten Flüssen

Die Tabelle 6.1 zeigt einen Vergleich der gemessenen turbulenten Flüsse von Impuls, fühlbarer und latenter Wärme mit den für diese Höhe berechneten Flüssen bei stationärer Rechnung. Da das Eddy-Kovarianzsystem nur Viertelstundenmittelwerte bestimmt, die mit einem Versatz von einer Viertelstunde gegenüber den verwendeten Modellterminen erhoben werden, sind die hier angegebenen „Meßwerte“ die arithmetischen Mittel der Viertelstundenmittelwerte vor und nach dem jeweiligen Modelltermin.

Vom Modell wird der Impulsfluß in 39 m Höhe gegenüber den Messungen fast durchgehend deutlich unterschätzt (betragsmäßig!). Eine gewisse zeitliche Korrelation zwischen modellierten und gemessenen Werten ist jedoch trotzdem erkennbar, da das Modell vormittags die höheren Flüsse berechnet als Nachmittag, genau wie vormittags höhere Flüsse gemessen wurden.

Beim fühlbaren Wärmefluß zeigt sich ein differenzierteres Bild. Während er in den Vormittagsstunden deutlich überschätzt wird, so unterschätzt ihn das Modell am Nachmittag zum Teil erheblich.

Wie der Impulsfluß, so wird auch der latente Wärmefluss gegenüber den Messungen während des ganzen Zeitraumes unterschätzt. 
Aus den durchgeführten stationären Rechnungen allein läßt sich mit Gewißheit keine Aussage darüber machen, ob der transiliente Schließungsansatz mit dem "split - timestep" Verfahren für stationäre Rechnungen geeignet ist. Einer Bewertung steht außerdem das Fehlen vom gemessenen Profilen der Eigenschaftsflüsse im Bestand entgegen.

Eine bessere Aussage über den Nutzen stationärer Modellrechnungen wird man nach der Betrachtung der instationären Modellrechnungen treffen können.

\subsection{Instationäre Modellrechnungen und Vergleich mit den Ergebnissen eines mit lokaler Turbu- lenzschließung arbeitenden Modells}

Für Vergleichsrechnungen stand ein instationäres atmosphärisches Grenzschichtmodell mit lokaler Turbulenzschließung (im folgenden K - Schließungsmodell) der Arbeitsgruppe Mix (1994) (siehe [26]) zur Verfügung. Um die Berechnungsergebnisse beider Modelle vergleichen zu können, wurden für den Energieaustausch des Systems Vegetation - Erdoberfläche - Atmosphäre, den Wasser- und Wärmetransport im Boden und die Beeinflussung des Strömungsfeldes in das K - Schließungsmodell die gleichen Ansätze eingebaut, wie sie auch im transilienten Grenzschichtmodell verwendet werden. Auch die Höhen der Modellschichten entsprechen denen des K-Schließungsmodells.

Ein Vergleich beider Modelle kann Aufschluß über die Vor- und Nachteile beider Schließungsansätze bei der Modellierung einer atmosphärischen Grenzschicht mit Waldbestand liefern. Aus diesem Grunde wurden instationäre Rechnungen (Tagesgang) mit beiden Modellen für den 20.06.1998 durchgeführt. Besonderes Augenmerk galt auch hier wieder den berechneten turbulenten Flüssen. Die Abbildung 6.5 zeigt die mit dem K-Modell berechneten Profile der horizontalen Windgeschwindigkeit (oben) sowie die mit dem instationären transilienten Modell berechneten Profile (unten) im Tagesverlauf im Vergleich mit den gemessenen Daten.

Die horizontale Windgeschwindigkeit wird vom K - Schließungsmodell in allen Höhen überschätzt. Dagegen wird die in den Messungen erkennbare zeitliche Variation der Windgeschwindigkeit, insbesondere die relativ starke Variation direkt über dem Kronendach, kaum widergegeben. Dem in den Messungen um 10:30 Uhr und 14:30 Uhr erkennbaren leichten sekundären Windmaximum in etwa 7 bis $8 \mathrm{~m}$ Höhe entspricht in den Modellergebnissen eine Höhenkonstanz bzw. leichte Zunahme der Windgeschwindigkeit mit der Höhe. Auch die am 21.06.1998 gemessenen höheren Windgeschwindigkeiten $(2.5 \mathrm{~m} / \mathrm{s}$ um 10:30 Uhr in $35 \mathrm{~m}$ Höhe - die Messungen zeigen ebenfalls ein angedeutetes sekundäres Windmaximum im Stammraum) führten zu keiner Berechnung eines solchen Maximums durch das K - Schließungsmodell.

Für die vom transilienten Modell berechneten Profile der horizontalen Windgeschwindigkeit im Bestand muß zunächst angemerkt werden, daß trotz vielfälti- 

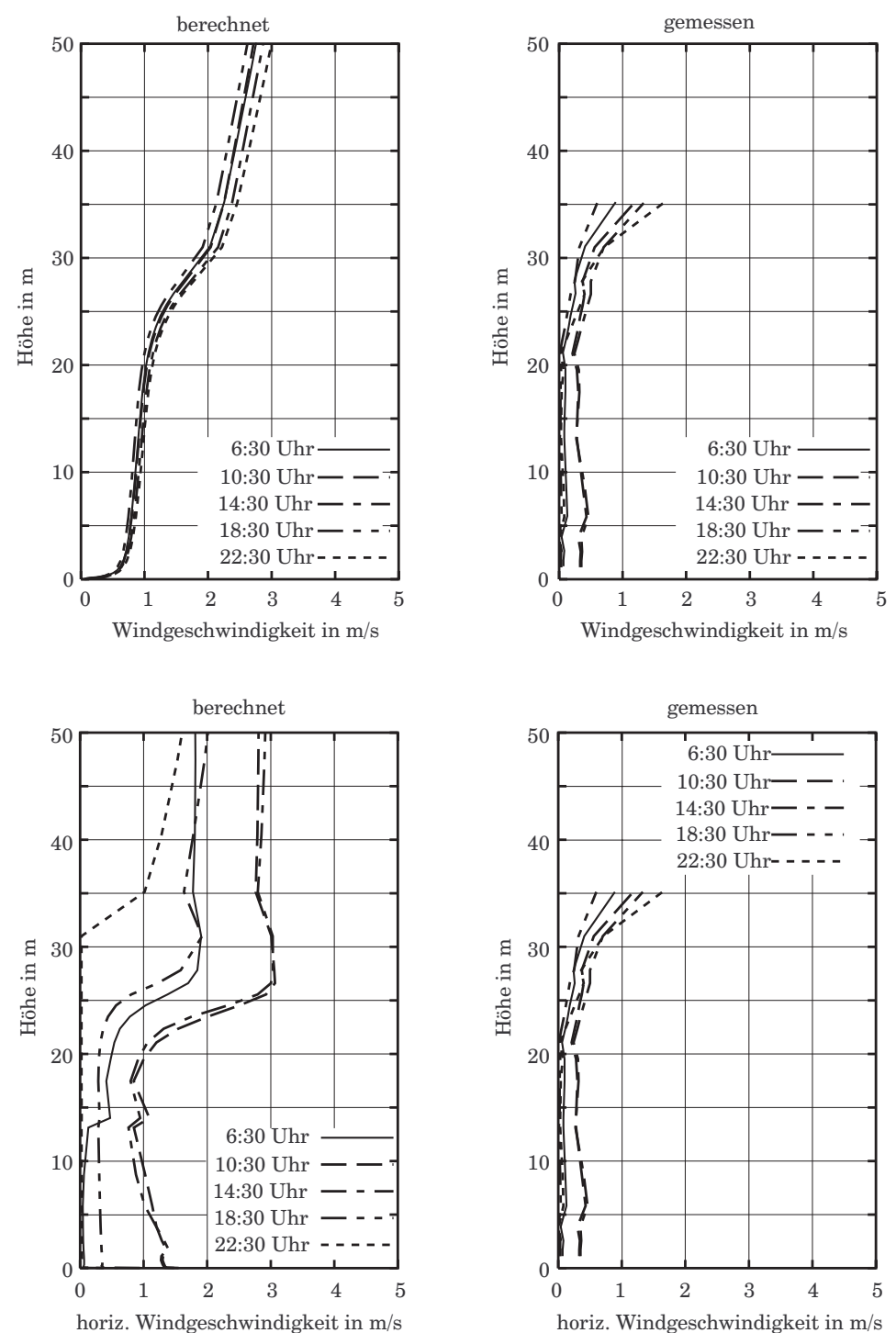

Abbildung 6.5: Mit dem instationären K - Modell (lokale Turbulenzschließung - oben) und mit dem transilienten Modell (transiliente Turbulenzschließung - unten) berechnete Profile der horizontalen Windgeschwindigkeit im Bestand für den 20.06.1998 im Vergleich mit den Meßwerten 
ger Bemühungen immer noch Probleme bei der Modellierung der horizontalen Windgeschwindigkeit bestehen, die nur mit einem modellinternen Fehler bei der Berechnung der Impulssenken im Bestand erklärt werden könnten. So wird über dem Kronendach eine Abnahme der horizontalen Windgeschwindigkeit berechnet, die physikalisch nicht erklärt werden kann. Dieser Effekt tritt jedoch nur dann auf, wenn bedingt durch die thermischen Schichtungsverhältnisse tagsüber starke vertikale turbulente Umlagerungen stattfinden. So tritt er um 22:30 Uhr bei einer modellierten starkstabilen Temperaturschichtung (Abb. 6.5) im selben Höhenbereich (die ebenfalls in den Messungen nicht beobachtet wurde) nicht mehr auf. Dabei muß gesagt werden, daß nicht klar ist, ob der "Defekt" im Temperaturprofil den "Defekt" im Windprofil verursacht oder umgekehrt.

Ansonsten überschätzt das transiliente Modell die Windgeschwindigkeiten im Bestand teilweise noch stärker als das K - Schließungsmodell, wobei es im Gegensatz zu diesem auch die tageszeitliche Variation deutlich überschätzt. Im Stammraum wird zwar ein sekundäres Windmaximum modelliert (Profile um 10:30 und 14:30), es wird jedoch zu stark widergegeben, was auch daran liegen könnte, daß die zu erwartende Abnahme der Windgeschwindigkeit zur Erdoberfläche hin auch nicht ansatzweise modelliert wird.

Die Abbildungen 6.6 und 6.7 zeigen die von beiden Modellen berechneten Profile der Lufttemperatur und der spezifischen Feuchte für den 20. Juni 1998 im Vergleich mit den Messungen.

Mit dem K - Schließungsmodell wird die Lufttemperatur allen 3 ausgewerteten am besten widergegeben. Richtig erfaßt wird die Amplitude des Tagesganges der Lufttemperatur im Bestand von etwa $5 \mathrm{Grad}$, sowie die angedeutete stabile Schichtung in unmittelbarer Bodennähe. Auch das Temperaturmaximum im Kronenraum um die Mittagszeit wird widergegeben. Dagegen fällt die abendliche Abkühlung der Luft etwas stärker aus, als es die Messungen zeigen. So ist die Lufttemperatur im Kronenraum um 18:30 Uhr gegenüber 14:30 schon wieder um etwa 2 Grad gesunken, obwohl die Messungen in etwa gleichgebliebene Werte anzeigen.

Auch vom transilienten Modell werden die gemessenen Temperaturprofile mit Ausnahme des schon erwähnten unrealistischen Temperaturprofiles um 22:30, für die eine Erklärung noch fehlt, gut widergegeben. Trotzdem sind die Differenzen zwischen den modellierten und gemessenen Temperaturwerten größer als beim K - Schließungsmodell. So wird das Temperaturmaximum im Höhenprofil um 10:30 und 14:30 nicht im Kronenraum, wie es die Messungen zeigen, sondern im oberen Stammraum widergegeben. Direkt über der Erdoberfläche wird im Gegensatz zu der in den Messungen angedeuteten und vom K - Schließungsmodell berechneten stabilen Temperaturschichtung, vom transilienten Modell immer eine labile Temperaturschichtung modelliert. Allerdings ist es auch durchaus möglich und aufgrund der Erwärmung der Erdoberfläche auch physikalisch begründbar, daß in den unmittelbar über der Erdoberfläche liegenden Atmosphärenschichten, für die keine regelmäßigen Temperaturmeßwerte vorliegen, an diesem Tag eine labile Tenperaturschichtung vorlag, so daß sich in diesem Punkt keine Aussage darüber machen läßt, welches der beiden Modelle die Realität besser widergibt. 
Bei der spezifischen Feuchte konnten aus den vorliegenden Messungen der relativen Feuchte leider nur Werte im Höhenbereich von $9 \mathrm{~m}$ bis $21 \mathrm{~m}$ Höhe berechnet werden, so daß umgerechnet auf die Höhen der Modellschichten nur Profile von $9 \mathrm{~m}$ bis etwa $19 \mathrm{~m}$ im Bestand als "Meßwerte“ zur Verfügung standen. Bei einem Vergleich der modellierten Werte des K-Schließungsmodells mit den Messungen fällt zunächst auf, daß die Werte im allgemeinen bis zu $5 \mathrm{~g} / \mathrm{kg}$ (14:30 Uhr) überschätzt werden. Die Abnahme der Werte mit der Höhe vom Stamm- zum Kronenraum wird nicht widergegeben. Auch die zeitliche Zuordnung der Tagesmaximal- und Tagesminimalwerte wird nicht richtig modelliert. So wurden die niedrigsten Werte um 14:30 gemessen, erscheinen im Modell aber um 6:00 Uhr, die höchsten Werte wurden um 22:30 gemessen, werden vom Modell aber um 18:30 widergegeben. Die vom transilienten Modell berechneten Werte der spezifischen Luftfeuchte zeigen beim Übergang vom Stamm- in den Kronenraum, wie auch die vom K - Modell berechneten Werte, keine generelle Abnahme mit der Höhe, wie sie die gemessenen Profile aufweisen. Um 14:30 Uhr zeigt sich im Stammraum zwischen etwa 5 und 18 m sogar eine Zunahme der spezifischen Feuchte mit der Höhe. Über die berechneten Profile oberhalb $20 \mathrm{~m}$ Höhe läßt sich keine Vergleichsaussage mit Meßwerten anstellen da in diesem Bereich keine Werte der spezifischen Feuchte vorliegen. Eine Abnahme der spezifischen Feuchte mit der Höhe im oberen Kronenraum läßt sich aber aufgrund der fehlenden Feuchtequelle für die Luft über dem Kronendach und der mit der Höhe abnehmenden Nadeloberfläche in diesem Bereich und der somit geringeren Feuchtezufuhr durch Evapotranspiration begründen. Wie bei den Messungen werden in allen die höchsten Werte der spezifischen Feuchte vom Modell in den Nachtstunden (22:30 Uhr) ausgegeben. Dagegen wird das gemessene deutliche Minimum um 14:30 Uhr nicht widergegeben.

Bei dem durchgeführten Vergleich der berechneten Profile der horizontalen Windgeschwindigkeit, der Lufttemperatur und der spezifischen Feuchte des K - Schließungsmodells bzw. des transilienten Modells mit den Meßwerten entsteht im allgemeinen der Eindruck als sei das K - Schließungsmodell besser in der Lage, diese Größen zu modellieren. Zugunsten des transilienten Modells muß allerdings gesagt werden, daß es noch offensichtliche Modellierungseffekte bzw. - defekte gibt, die noch gelöst werden müssen, wobei unklar ist, ob es sich um spezifische Probleme des transilienten Turbulenzschließungsansatzes handelt oder um Fehler bei der Implementierung desselben in ein atmosphärisches Grenzschichtmodell mit Waldbestand. Es scheint andererseits, als sei das K - Schließungsmodell nicht in der Lage, das in den Messungen angedeutete sekundäre Windmaximum im Stammraum widerzugeben.

Für eine Bewertung beider Schließungsansätze reicht ein Vergleich der modellierten Profile luftgetragener konservativer Eigenschaften nicht aus. Für die Ökosystemforschung sind vielmehr die modellierten vertikalen turbulenten Eigenschaftsflüsse von Interesse (siehe Einleitung). Deshalb ist ein Vergleich der modellierten Eigenschaftsflüsse mit den Messungen von grundlegender Bedeutung für die Bewertung unterschiedlicher Turbulenzschließungsansätze in Bezug auf die Modellierung physikalischer Prozesse in Ökosystemen. 

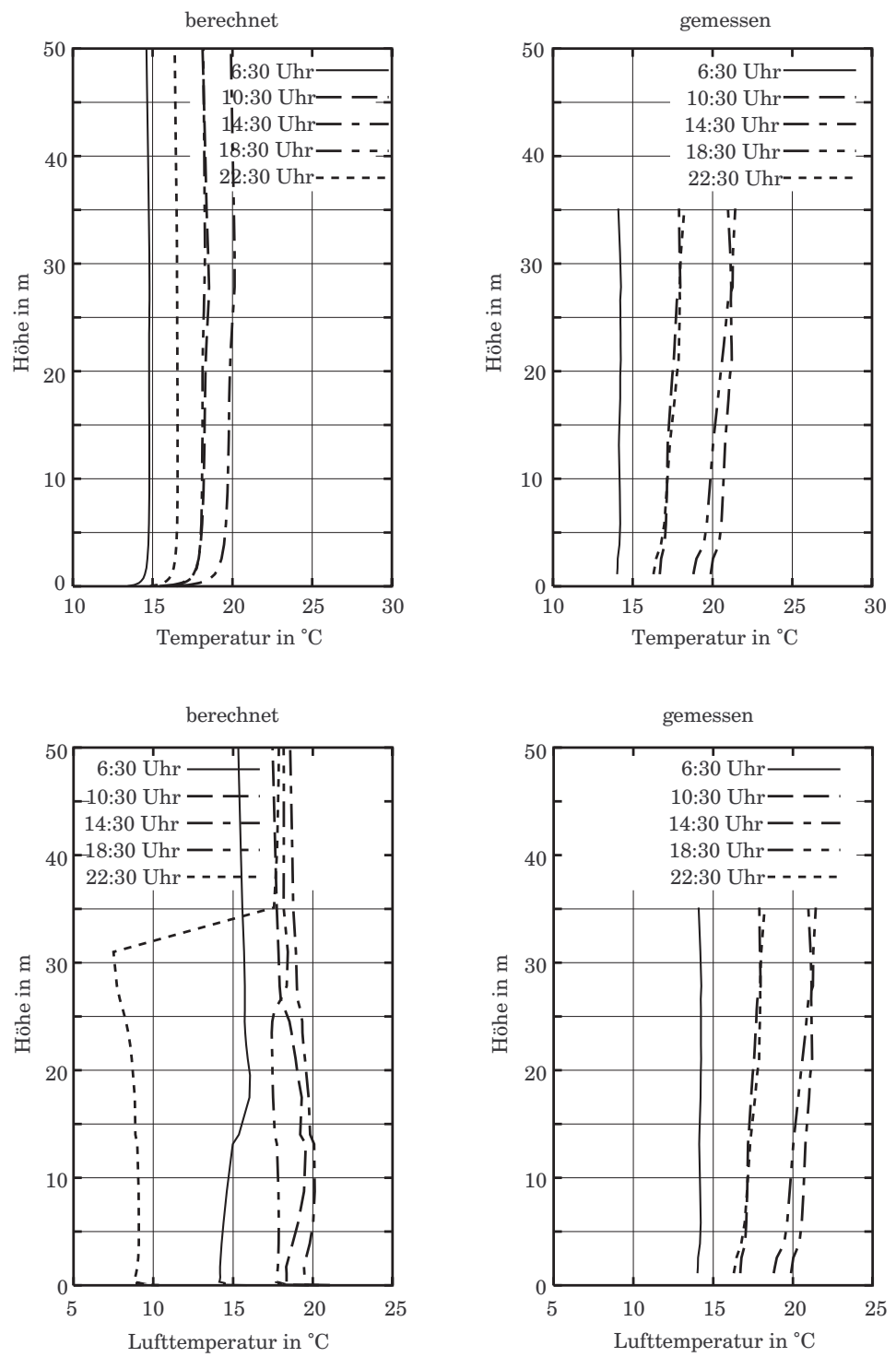

Abbildung 6.6: Mit dem instationären K - Modell (lokale Turbulenzschließung - oben) und mit dem transilienten Modell (transiliente Turbulenzschließung - unten) berechnete Profile der Lufttemperatur im Bestand für den 20.06.1998 im Vergleich mit den Meßwerten 

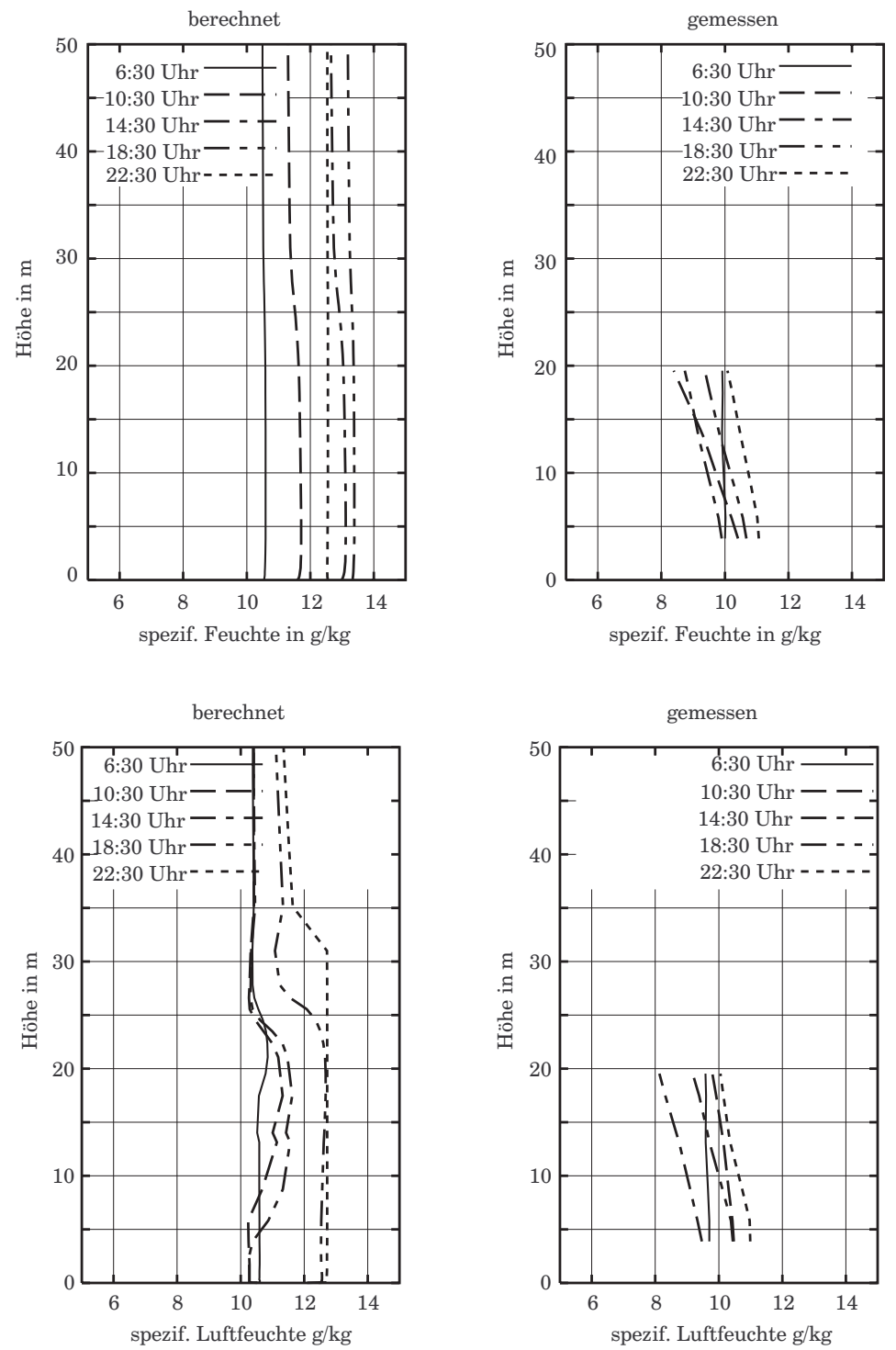

Abbildung 6.7: Mit dem instationären K - Modell (lokale Turbulenzschließung - oben) und mit dem transilienten Modell (transiliente Turbulenzschließung - unten) berechnete Profile der spezifischen Feuchte im Bestand für den 20.06.1998 im Vergleich mit den Meßwerten 

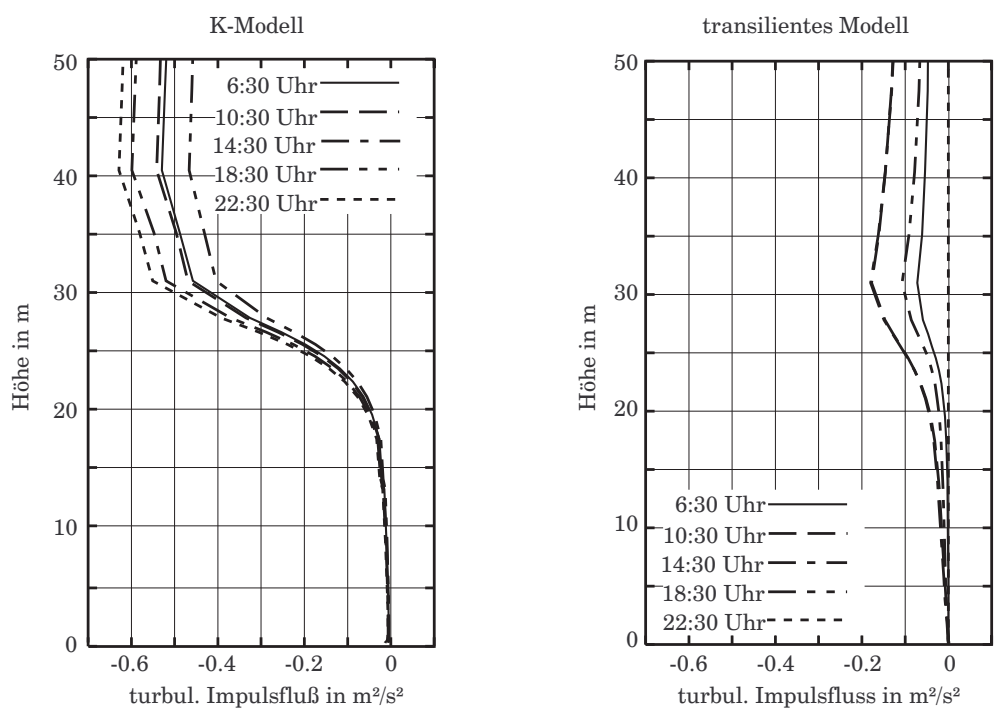

Abbildung 6.8: Mit dem instationären K-Modell (lokale Turbulenzschließung - links) und dem instationären transilienten Modell (transiliente Turbulenzschließung - rechts) berechnete turbulente Impulsflüsse für den 20.06.1998 im Bestand

Leider liegen für die 2 ausgewählten Tage keine Vertikalprofile der turbulenten Flüsse vor, so daß nur die mittels Eddy-Kovarianzverfahren in 39 m Höhe gemessenen Flüsse für einen Vergleich mit Meßwerten zur Verfügung stehen. Deshalb sollen zuerst die Profile der von beiden Modellen berechneten Flüsse verglichen werden, dann folgt ein Vergleich des zeitlichen Verlaufes der von beiden Modellen berechneten Flüsse in 39 m Höhe und der Meßwerte.

Die Abbildungen 6.6 und 6.6 zeigen dazu zunächst die vom K - Schließungsmodell und vom transilienten Modell berechneten turbulenten Impulsflüsse (auf die Masseneinheit bezogen, deshalb die Einheit $\mathrm{m}^{2} / \mathrm{s}^{2}$ ) und fühlbaren Wärmeflüsse im Bestand für den 20. Juni 1998. Die berechneten Werte der turbulenten Impulsflüsse sind durchgehend negativ (abwärtsgerichteter Impulstransport), da die Erdoberfläche und der Kronenraum als Impulssenken für die atmosphärische Strömung wirken. Die Impulsflüsse zeigen bei beiden Modellen im Stammraum die betragsmäßig niedrigsten Werte, wie es auch aufgrund der geringen gemessenen Windgeschwindigkeiten und vor allem aufgrund der geringen vertikalen Windscherung in diesem Bereich zu erwarten ist. Allgemein wird aber vom K - Schließungsmodell ein stärkerer abwärtsgerichteter Impulstransport simuliert als vom transilienten Modell.

Da die vom K - Schließungsmodell simulierten Windprofile im Bestand kein sekundäres Windmaximum aufweisen, kann bei den von diesem Modell berechneten Impulsflüssen kein Vorzeichenwechsel, d.h. kein Richtungswechsel der Flüsse auftreten. Im Falle eines sekundären Windmaximums wäre dieser Richtungswechsel zu erwarten, da die Flüsse beim K - Schließungsansatz immer in Richtung des meteorologischen Gradienten gerichtet sind. 

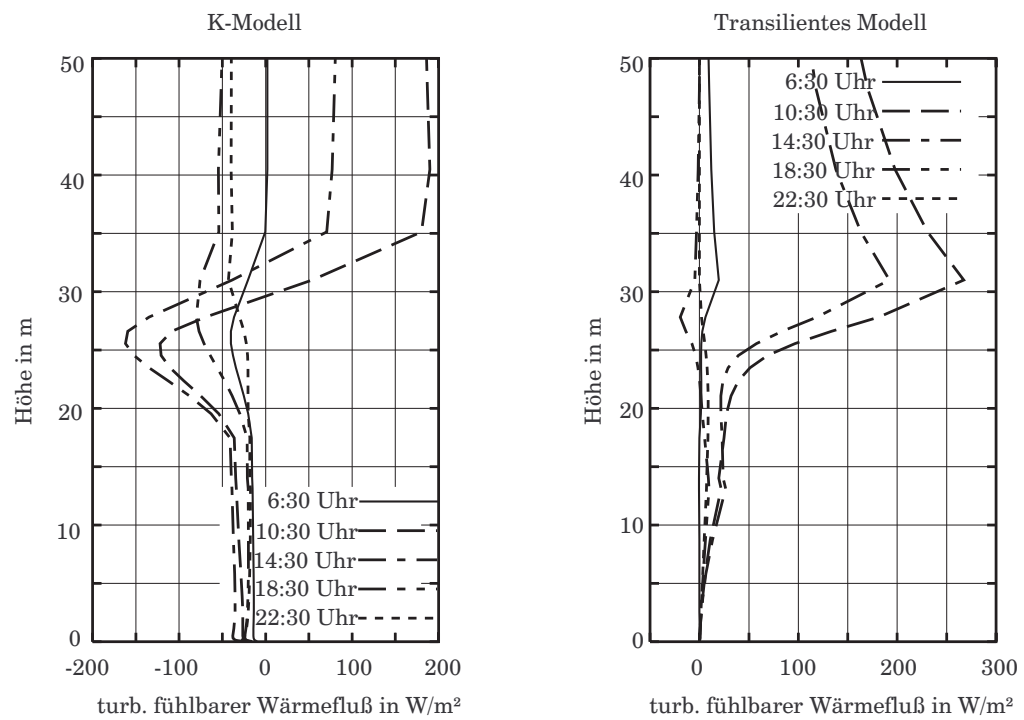

Abbildung 6.9: Mit dem instationären K-Modell (lokale Turbulenzschließung - links) und dem instationären transilienten Modell (transiliente Turbulenzschließung - rechts) berechnete turbulente Flüsse fühlbarer Wärme für den 20.06.1998 im Bestand

Bei den von beiden Modellen berechneten Impulsflüssen zeigt sich auch eine Zunahme der turbulenten Impulstransporte im Kronenraum gegenüber dem Stammraum, beim transilienten Modell fällt diese Zunahme jedoch sehr viel geringer aus.

Das K - Schließungsmodell modelliert über dem Bestand einen Bereich relativ konstanter Impulsflüsse (Prandtl-Schicht). Über dem Fichtenbestand im Solling wurde die Existenz einer Schicht höhenkonstanter Flüsse im Höhenbereich von 39 - 45 m nachgewiesen ([23],[24]), ein Bereich der sich mit den Berechnungen dieses Modells deckt. Obwohl keine Meßwerte im Profil vorliegen, kann man also sagen, das daß K-Modell zumindest in diesem Bereich charakteristische, über dem Bestand zu beobachtende Effekte bezüglich der turbulenten Flüsse widergeben kann.

Das transiliente Modell berechnet die Prandtl-Schicht weniger deutlich. Direkt über dem Bestand ist im Gegensatz zum K - Schließungsmodell schon wieder eine Abnahme der Impulstransporte zu erkennen.

Trotz des vom transilienten Modell berechneten sekundären Windmaximums im Stammraum wird in diesem Höhenbereich vom transilienten Modell kein Vorzeichenwechsel des Impulsflusses simuliert. Aus dieser Tatsache folgt, daß das transiliente Modell im Stammraum in der Lage ist Impulsflüsse gegen den lokalen meteorologischen Gradienten (counter gradient fluxes) zu modellieren, da in diesem Höhenbereich der meteorologische Gradient des horizontalen Impulses nach oben gerichtet ist. Leider läßt sich nicht mit Messungen der Impulsflüsse innerhalb des Bestandes belegen, ob an diesem Tag tatsächlich "Counter gradient" - Flüsse aufgetreten sind. 
Die von beiden Modellen berechneten turbulenten Flüsse fühlbarer Wärme zeigen trotz der geringen Höhenvariation der Lufttemperatur eine relativ starke Variation mit der Höhe, aber auch einen ausgesprochenen Tagesgang über dem Bestand. Ansonsten zeigen sich zwischen den Ergebnissen beider Modelle größere Unterschiede.

Über dem Bestand wird vom K - Schließungsmodell nur in den Vormittagsstunden ein aufwärtsgerichteter Transport fühlbarer Wärme modelliert, während der Transport nachmittags und nachts abwärts gerichtet ist. Das transiliente Modell berechnet dagegen nur um 18:30 Uhr zwischen 20 und 35 m Höhe einen deutlich abwärtsgerichteten Wärmestrom. Tagsüber sind auch hier die turbulenten Flüsse fühlbarer Wärme aus dem Bestand heraus gerichtet. In beiden Modellen wirkt der Bestand also als Wärmequelle für die Atmosphäre.

Deutliche Unterschiede zwischen beiden Modellen zeigen sich bei den modellierten fühlbaren Wärmeflüssen im Kronenraum. Während das transiliente Modell bis auf die Ausnahme um 18:30 Uhr immer aufwärtsgerichtete Wärmetransporte zwischen 20 und $40 \mathrm{~m}$ Höhe angibt, kommt es beim K - Schließungsmodell in den Vor- und frühen Nachmittagsstunden (10:30 und 14:30) zu einem Richtungswechsel der Transporte im Bereich von 30 bis $35 \mathrm{~m}$. Unterhalb dieser Höhe sind die Flüsse durchgehend abwärts gerichtet. In etwa $27 \mathrm{~m}$ Höhe werden die maximalen abwärtsgerichteten Transporte erreicht. Dieser Effekt kann nur durch das sehr geringe Temperaturmaximum im oberen Kronenraum erklärt werden und zeigt somit, wie stark das K - Schließungsmodell auf sehr kleine Unterschiede der thermischen Schichtung reagiert und das die damit berechneten turbulenten Flüsse immer in Richtung des meteorologischen Gradienten fließen.

Für den Bereich zwischen 2 und 10 m Höhe im Stammraum, in dem vom transilienten Modell eine Temperaturzunahme mit der Höhe modelliert wird (Abb. 6.6), werden von diesem Modell aufwärtsgerichtete turbulente Wärmeflüsse prognostiziert, also Flüsse gegen den lokalen Gradienten. Eine mögliche physikalische Erklärung liegt darin, daß durch die Erwärmung der Erdoberfläche auch die der Erdoberfläche unmittelbar aufliegende Luftschicht, welche vom Modell im Temperaturprofil nicht mehr aufgelöst wird, erwärmt wird, und daß dadurch warme Luftpakete aufgrund von thermischem Auftrieb aufsteigen, deren Impuls ausreichend ist, um den Bereich der Temperaturinversion zu überwinden. Der dadurch erfolgende Transport von Wärme führt zu den modellierten "counter gradient" - Flüssen. Die Prandtl-Schicht oberhalb des Bestandes mit ihren charakteristischen höhenkonstanten Flüssen, ist im Gegensatz zu den vom K - Schließungsmodell berechneten Wärmeflüssen nicht erkennbar. Der Betrag der aufwärtsgerichteten Flüsse über dem Bestand wird etwa doppelt so hoch prognostiziert, als beim K - Schließungsmodell. Die Abbildung 6.6 zeigt die von beiden Modellen berechneten Flüsse latenter Wärme im Bestand für den 20. Juni 1998 im Vergleich mit den Messungen.

Die von beiden Modellen berechneten latenten Wärmeflüsse zeigen ähnlich wie die Impulsflüsse und fühlbaren Wärmeflüsse im Stammraum sehr niedrige Beträge, die dann aber im Kronenraum mit der Höhe zunehmen und ihr Maximum im Bereich der Kronenobergrenze erreichen. Die erreichten Werte werden vom K - Modell jedoch mit maximal $300 \mathrm{~W} / \mathrm{m}^{2}$ deutlich höher als vom transilienten Modell $\left(120 \mathrm{~W} / \mathrm{m}^{2}\right)$ angegeben. 

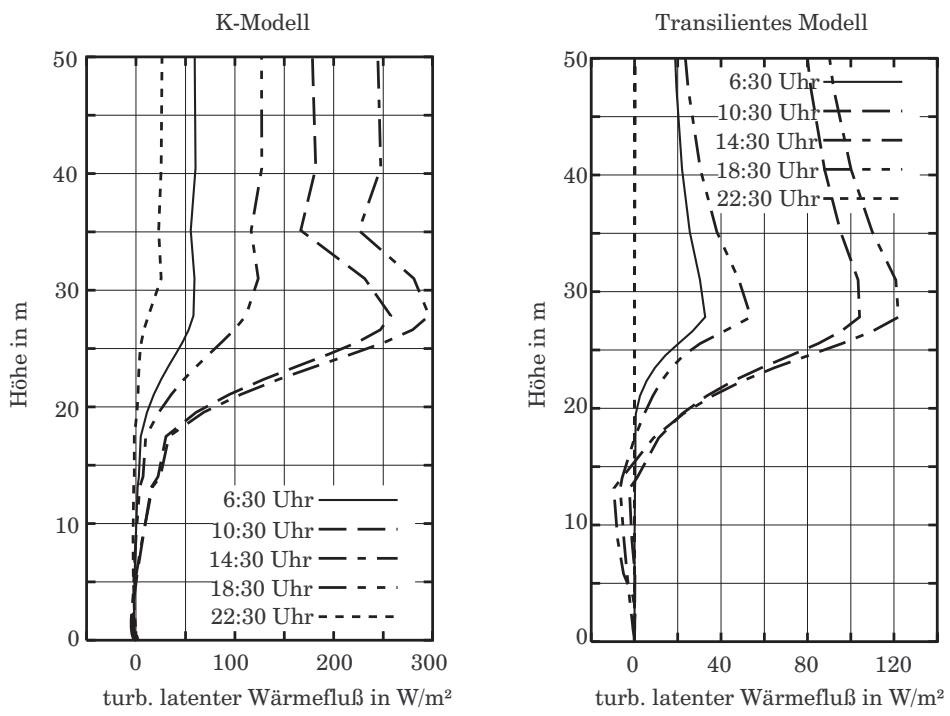

Abbildung 6.10: Mit dem instationären K-Modell (lokale Turbulenzschließung - links) und dem instationären transilienten Modell (transiliente Turbulenzschließung - rechts) berechnete turbulente Flüsse latenter Wärme für den 20.06.1998 im Bestand

Im Gegensatz zu den fühlbaren Wärmeflüssen sind die latenten Wärmeflüsse im Stammraum beim transilienten Modell unterhalb von $15 \mathrm{~m}$ durchweg abwärtsgerichtet, was beim K - Modell nicht auftritt. Da aber auch der lokale Gradient der Feuchteprofile nach unten gerichtet ist (Abb. 6.7), werden keine latenten Wärmeflüsse gegen den lokalen Gradienten prognostiziert.

Wie auch schon bei den Impuls- und fühlbaren Wärmeflüssen tritt auch bei den latenten Wärmeflüssen die Prandtl-Schicht über dem Bestand beim transilienten Modell weniger deutlich zu tage, als beim K - Schließungsmodell.

Nachdem die Profile der von beiden Modellen berechneten turbulenten Eigenschaftsflüsse verglichen wurden, soll nun der zeitliche Verlauf der berechneten Flüsse in 39 m Höhe mit den mittels Eddy-Kovarianzverfahren in dieser Höhe bestimmten Flüssen verglichen werden (Abb. 6.11 und 6.12). Die in den Grafiken dargestellten gemessenen Werte sind Viertelstundenmittelwerte, die vom Meßsystem automatisch gebildet werden. Die Abb. 6.11 zeigt oben einen Vergleich der von beiden Modellen berechneten mit den gemessenen Impulsflüssen. Der vom K - Schließungsmodell berechnete Impulsfluß liegt betragsmäßig deutlich über den gemessenen Werten, d.h. es wird über dem Bestand ein sehr viel stärkerer nach unten gerichteter Impulstransport berechnet, als es die Messungen zeigen. Die vom transilienten Modell berechneten Impulsflüsse stimmen qualitativ prinzipiell mit den gemessenen Werten überein. Aber auch hier zeigen sich im Tagesverlauf größere Abweichungen. So wird bis etwa 8:00 Uhr der abwärtsgerichtete Impulstransport deutlich unterschätzt und ab 20:00 Uhr verschwindet er völlig. Anscheinend wird die Unterbindung des vertikalen Austausches durch den Kronenraum in den Nacht- 

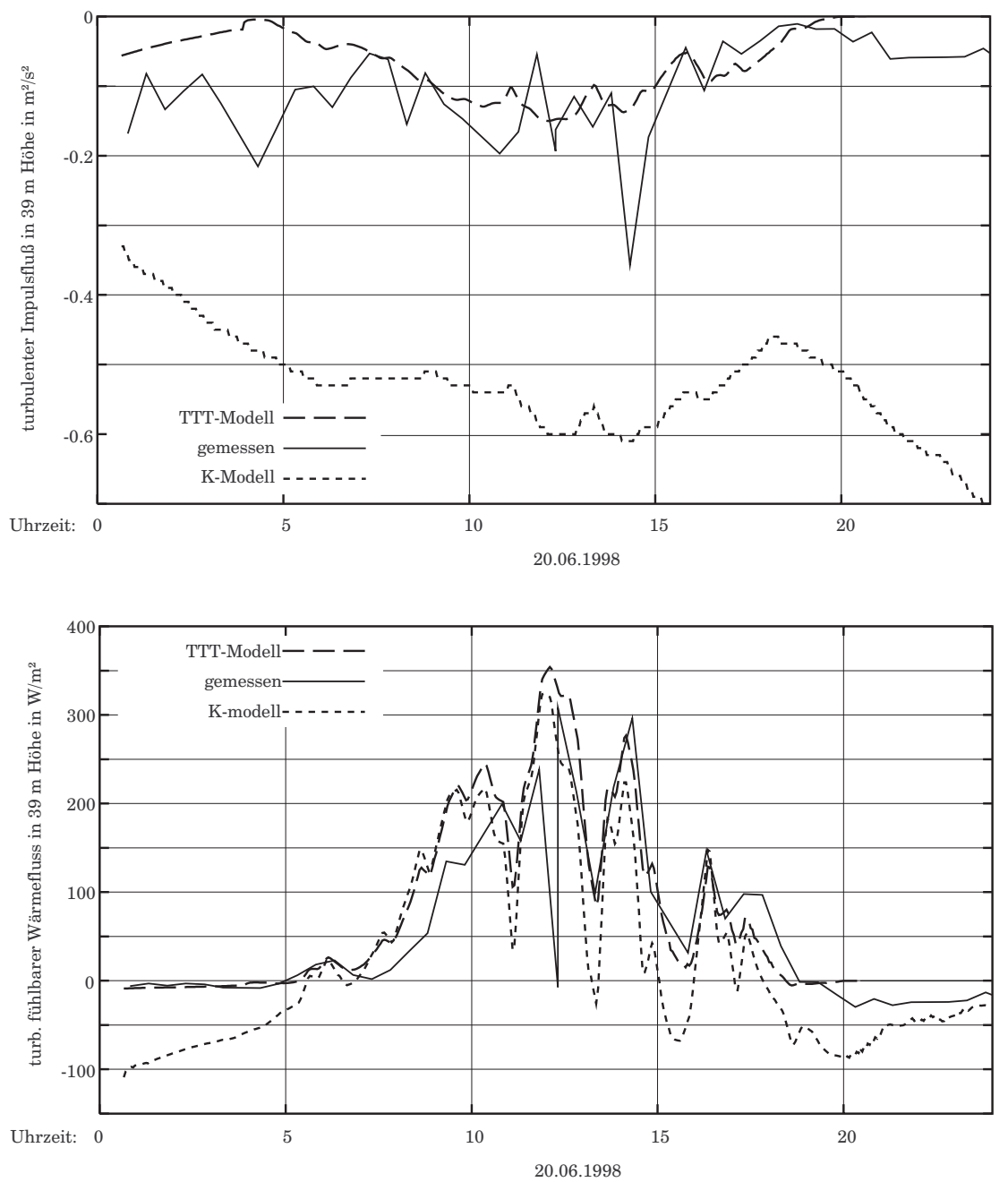

Abbildung 6.11: Vergleich der mittels 2 Modellen (lokale Turbulenzschließung (K - Modell) und transiliente Turbulenzschließung (TTT - Modell)) berechneten und der mittels Eddy-Kovarianzverfahren bestimmten Impulsflüsse (oben) und fühlbaren Wärmeflüsse (unten) in 39 m Höhe für den 20.06.1998 


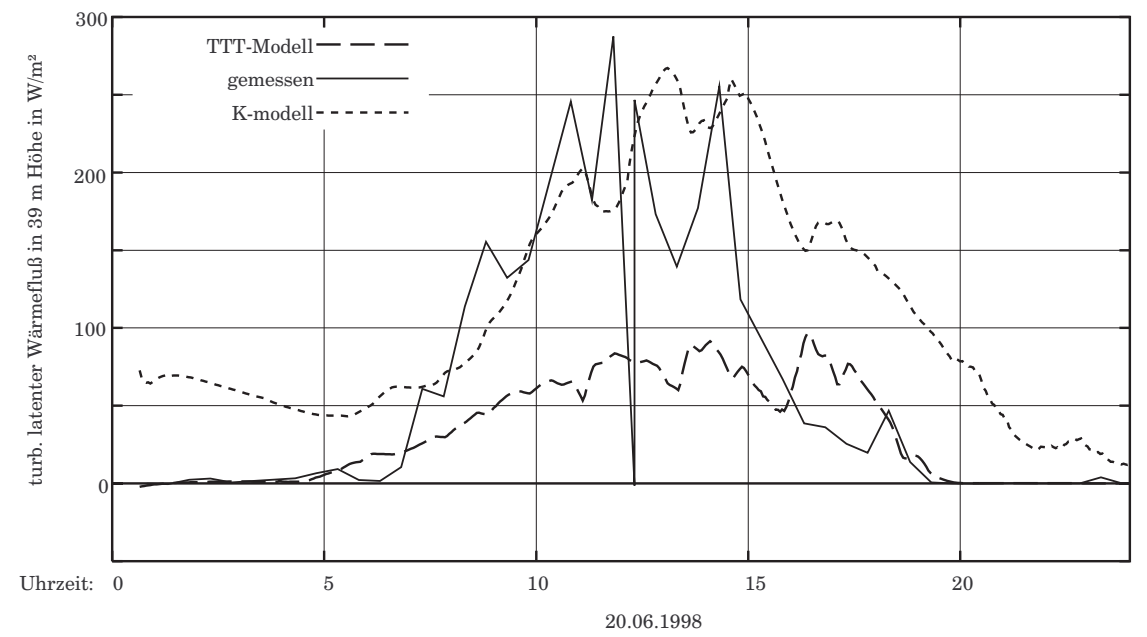

Abbildung 6.12: Vergleich der mittels 2 Modellen (lokale Turbulenzschließung (K - Modell) und transiliente Turbulenzschließung (TTT - Modell)) berechneten und der mittels Eddy-Kovarianzverfahren bestimmten Flüsse latenter Wärme in $39 \mathrm{~m}$ Höhe über dem Bestand für den 20.06.1998

stunden überschätzt.

Die von beiden Modellen berechneten fühlbaren Wärmeflüsse (Abb. 6.11 unten) stimmen im allgemeinen besser mit den Messungen überein, als es für die Impulsflüsse der Fall ist. Auch die zeitliche Zuordnung der Maximal- und Minimalwerte ist für beide Modelle recht gut. Wie auch schon beim Impulstransport werden vom $\mathrm{K}$ - Schließungsmodell die Wärmetransporte in den Nachtstunden (0:00-5:00 Uhr und 20:00 bis 24:00 Uhr) überschätzt. Dagegen scheint das transiliente Modell auch für die fühlbare Wärme die turbulenten Transporte in den Nachtstunden eher zu unterschätzen.

Größere Unterschiede zwischen den Ergebnissen beider Modelle und den Messungen zeigen sich wieder bei den latenten Wärmeflüssen. Während das K Schließungsmodell den latenten Wärmefluss meist überschätzt, wird er vom transilienten Modell auch am Tage deutlich unterschätzt. Nur am frühen Morgen (6:00 Uhr) und am späten Nachmittag (16:00 Uhr) werden die Flüsse vom transilienten Modell überschätzt.

Da beide Modelle bis auf den Turbulenzschließungsansatz die gleichen Parametrisierungen der im Waldbestand ablaufenden physikalischen Prozesse (dieselbe Modellphysik) enthalten, ist der Grund für die je nach Eigenschaft so unterschiedlichen Ergebnisse im Vergleich zu den Messungen nicht sofort ersichtlich. Eine mögliche Erklärung wäre die unterschiedliche Höhe der sich modellintern bildenden atmosphärischen Grenzschicht. Da sich insbesondere die fühlbare und latente Wärme vor allem in der Grenzschicht ausbreiten, würde zum Beispiel eine zu hoch angenommene Grenzschicht bei gleicher Quell- bzw. Senkenstärke geringere lokale Werte einer Eigenschaft zur Folge haben. Andererseits kann sich dadurch auch der vertikale Gradient einer Ei- 
genschaft und damit auch dessen vertikaler Fluß ändern.

Deshalb sollen im folgenden als Abschluß eines Modellvergleiches beider Schließungsansätze die Unterschiede der berechneten Höhe der atmosphärischen Grenzschicht betrachtet werden (Abb. 6.6). Die berechnete Spurenstoffkonzentration in Erdbodennähe wird auch von der berechneten Höhe der atmosphärischen Grenzschicht abhängen. Als Kriterium für die Grenzschichthöhe wurde anhand des charakteristischen Aufbaus der Grenzschicht (siehe Einleitung) das absolute Minimum des fühlbaren turbulenten Wärmeflusses über dem Bestand verwendet. Meßwerte der atmosphärischen Grenzschicht standen für diesen Tag leider nicht zur Verfügung, so daß die Modellergebnisse bezüglich dieses Punktes nicht abschließend beurteilt werden können. Es zeigt sich jedoch, daß sich mit dem Kriterium des Wärmeflußminimums aus den Modellergebnissen des K-Modells eine Grenzschichthöhe sicherer bestimmen läßt, als beim transilienten Modell, wie man anhand der starken Variation der Werte sehen kann. Das Wachstum der Grenzschichthöhe in den Morgenstunden setzt beim transilienten Modell später ein, erfolgt jedoch schneller als beim KModell. Nimmt man für die stark streuenden Werte des transilienten Modells zwischen 10:00 und 15:00 Uhr einen mittleren Wert von $1400 \mathrm{~m}$ an (siehe eingezeichnete Kurve), so fällt die maximale Grenzschichthöhe beim transilienten Modell etwa 500 m höher aus als beim K-Schließungsmodell. Es fällt weiterhin

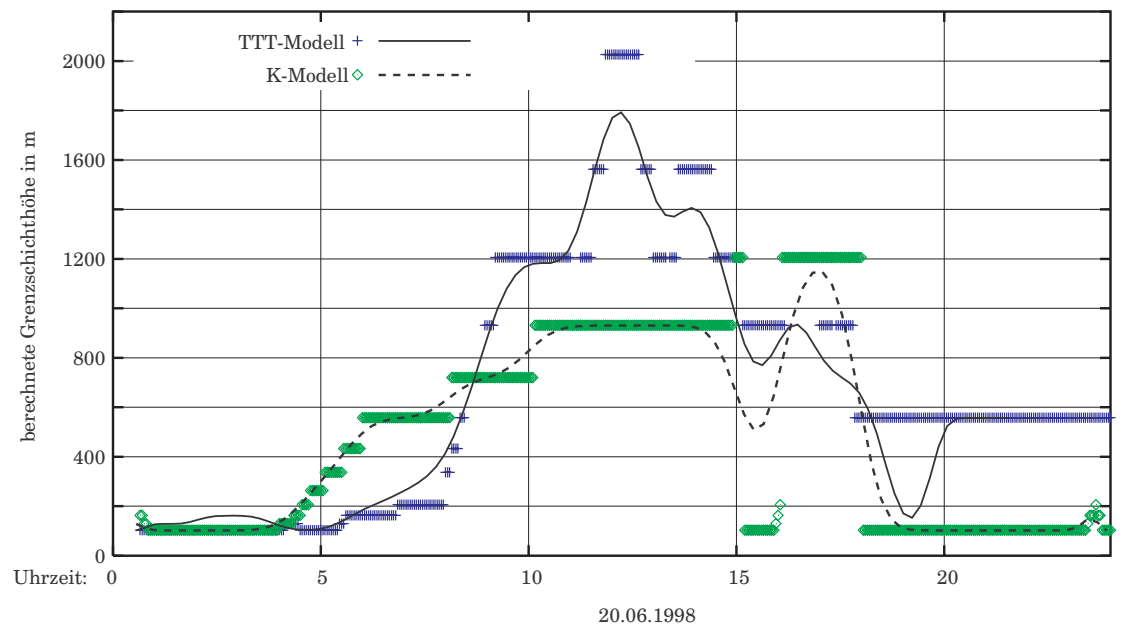

Abbildung 6.13: Vergleich der mittels 2 Modellen (lokale Turbulenzschließung (K-Modell) und transiliente Turbulenzschließung (TTT-Modell)) berechneten Tagesgänge der Grenzschichthöhe für den 20.06.1998

auf, daß das transiliente Modell im Gegensatz zum K - Modell für die Nacht vom 20. zum 21. Juni 1998 eine größere Höhe der atmosphärischen Grenzschicht prognostiziert als in der vorhergehenden Nacht. Leider standen keine Meßwerte (z.B. von hochauflösenden Radiosondenaufstiegen) zur Verfügung aus denen man die tatsächliche Grenzschichthöhe hätte ablesen können, so daß nicht mit absoluter Sicherheit gesagt werden kann welches Modell in diesem Punkt realistischere Ergebnisse liefert. 


\section{Kapitel 7}

\section{Zusammenfassung}

Messungen belegen, daß bei der Durchströmung von Waldbeständen im Stammraum neben den von der Umströmung der Pflanzenelemente herrührenden Wirbeln auch Wirbel von der Größenordnung des Bestandes auftreten, deren Transporte nicht vernachlässigt werden können. Deshalb muß ein Modell zur Beschreibung des atmosphärischen Austausches in und über Waldbeständen in der Lage sein, auch die Effekte dieser Wirbel zu berücksichtigen. Dazu bieten sich nichtlokale Turbulenzschließungsverfahren an.

Das im Rahmen dieser Arbeit zur Modellierung atmosphärischer Eigenschaften bei der Strömung durch und über einen Fichtenbestand im Solling angewendete nichtlokale Turbulenzschließungsverfahren nach der transilienten Turbulenztheorie ist prinzipiell in der Lage, den turbulenten Austausch in und über einem Waldbestand im Solling qualitativ richtig widerzugeben. Dies haben die durchgeführten Modellrechnungen gezeigt. Probleme bereitet derzeit noch die realistische Widergabe der Windprofile in und knapp über dem Bestand. Trotz dieses Modell - „Defektes“ stimmen die modellierten Impulsflüsse in $39 \mathrm{~m}$ bei instationärer Rechnung sehr gut mit den Daten von Eddy - Kovarianzmessungen überein. Ein Vergleich mit den Modellergebnissen eines bei gleicher Modellphysik mit lokaler Turbulenzschließung (K - Schließung) arbeitenden Modells hat dies deutlich zutage gebracht. Obwohl die vom K - Schließungsmodell berechneten Profile atmosphärischer Eigenschaften (horizontale Windgeschwindigkeit, Lufttemperatur und spezifische Feuchte) in mancher Hinsicht besser mit den Messungen übereinstimmten, wurde für den 20. Juni 1998 zumindest der Impulsfluß in 39 m Höhe vom K - Schließungsmodell betragsmäßig stark überschätzt, während die vom transilienten Modell berechneten Werte besser mit den Messungen übereinstimmten. Die fühlbaren Wärmeflüsse werden von beiden Modellen in den instationären Rechnungen gut widergegeben. Bei den latenten Wärmeflüssen werden die Werte vom instationären transilienten Modell jedoch meist unterschätzt, während das KSchließungsmodell eher zu einer Überschätzung neigt.

Bei den berechneten Profilen der turbulenten Eigenschaftsflüsse von Impuls, fühlbarer und latenter Wärme hat sich gezeigt, daß das Modell auf der Basis der lokalen Turbulenzschließung erwartungsgemäß prinzipiell keine Flüsse gegen den lokalen Gradienten modellieren kann. Beim transilienten Schlie- 
ßungsansatz ist dies sehr wohl möglich. Insbesondere beim vertikalen Transport von Impuls ergibt sich eine prinzipielle Übereinstimmung mit dem in der Einleitung erwähnten negativen turbulenten Diffusionskoeffizienten im Stammraum als Analogon zu den Flüssen gegen den lokalen Gradienten.

Die erstmals mit dem transilienten Modell durchgeführten stationären Rechnungen haben prinzipiell schlechtere Ergebnisse geliefert, als die instationären Rechnungen. So werden die berechneten turbulenten Flüsse über dem Bestand für alle betrachteten Größen betragsmäßig zu allen Tageszeiten deutlich unterschätzt. Bei zukünftigen Berechnungen sollte daher trotz der bei einer Prognose, wie sie praktisch bei einer instationären Rechnung erstellt wird, zu erwartenden zeitlichen Divergenz zwischen modellierten und gemessenen Eigenschaftsprofilen, eher instationär gerechnet werden.

Eine Erweiterung des Modells auch auf andere atmosphärische Prozesse, z.B. $\mathrm{CO}_{2}$ - Flüsse wird zeigen, inwieweit sich die Modellergebnisse bei der Verwendung des transilienten Schließungsansatzes gegenüber dem K - Schließungsansatz verbessern. 


\section{Literaturverzeichnis}

[1] Bleyl, M. R., 1998: $\mathrm{CO}_{2}$ - Flußmessungen auf einer landwirtschaftlichen Nutzfläche und in einem Hochmoor mit Hilfe der Eddykorrelationsmethode, Diplomarbeit am Departement Geographie, Institut für Meteorologie, Klimatologie und Fernerkundung, Universität Basel, 57 S..

[2] Chrobok, G., 1988: Zur numerischen Simulation konvektiver Grenzschichten mit integralen Schließungsansätzen, Diplomarbeit am Fachbereich Physik, Hannover, $92 \mathrm{~S}$. .

[3] Cienciala, E., Eckersten, H., Lindroth, A., Hällgren, J.-E., 1994: Simulated and measured water uptake by Picea Abies under non-limiting soil water conditions, Agricul. For. Met., 71, 147 - 164.

[4] Constantin, J., 1993: Stoffeinträge in ein Fichtenwaldökosystem durch Deposition luftgetragener Partikel und Nebeltröpfchen, Dissertation am Fachbereich Physik, Universität Göttingen, 165 S..

[5] Constantin, J., Inclán, M. G., Raschendorfer, M., 1998: The energy budget of a spruce forest: field measurements and comparison with the forest-landatmosphere model (FLAME), J. Hydrol., 212-213, 22-35.

[6] Deardorff, J. W., 1970: Convective Velocity and Temperature Scales for the Unstable Planetary Boundary Layer and for Rayleigh Convection, J. Atmos. Sci. 27, 1211 - 1213.

[7] Deardorff, J.W., 1972: Parameterization of the planetary boundary layer for use in general circulation models, Mon. Wea. Rev., 100, 93 - 106.

[8] Deardorff, J.W., 1978: Efficient prediction of ground surface temperature and moisture with inclusion of a layer of vegetation, J. Geophys. Res., 83, 1889 1903.

[9] Detering, H.W., 1985: Mischungsweg und turbulenter Diffusionskoeffizient in atmosphärischen Simulationsmodellen, Berichte des Instituts für Meteorologie und Klimatologie der Universität Hannover, 211 Seiten.

[10] Ditzer, Th., 1994: Methodische Untersuchungen zur Wasserhaushaltsmodellierung im Rahmen der Ökosystemforschung, Diplomarbeit am Fachbereich Physik, Universität Gesamthochschule Kassel, 168 Seiten. 
[11] Ebert, E. E., Schumann, U. and Stull, R. B., 1989: Nonlocal turbulent mixing in the convective boundary layer evaluated from large-eddy simulation, J. Atmos. Sci. 46, 2178 - 2207.

[12] Ellenberg, H., Mayer, R., Schauermann, J., 1986: Ökosystemforschung - Ergebnisse des Sollingprojekts 1966-1986, Ulmer, Stuttgart, 507 S..

[13] Enquete Kommission "Schutz der Erdatmosphäre“ des Deutschen Bundestages (Herausgeber), 1992: Klimaänderung gefährdet globale Entwicklung, Economica Verlag, Bonn, 235 S..

[14] Feddes, R. A., Kowalik, P. J., Zaradny H., 1978: Simulation of field water use and crop yield, Simulation Monographs, Centre for Agricultural Publishing and Documentation, Wageningen, The Netherlands, 188 pp..

[15] Gisi, U. (Herausgeber und Mitverf.), Schenker, R., Schulin, R., Stadelmann F. X., Sticher, H., 1990: Bodenökologie, Georg Thieme Verlag Stuttgart New York, 304 Seiten.

[16] Gross, G., 1993: Numerical Simulation of Canopy Flows, Springer Series in physical environment 12, Springer-Verlag Berlin Heidelberg New York, $167 \mathrm{pp}$.

[17] Ibrom, A.,1993: Die Deposition und die Pflanzenauswaschung (Leaching) von Pflanzennährstoffen in einem Fichtenbestand im Solling, Berichte des Forschungszentrums Waldökosysteme, Reihe A, Bd. 105, Selbstverlag des Forschungszentrums Waldökosysteme der Universität Göttingen, 165 S..

[18] Inclán, M. G., Forkel, R. and Dlugi R., 1996: Application of Transilient Turbulent Theory to study interactions between the atmospheric boundary-layer and forest canopies, Boundary-Layer Meteorol., 79, 315 - 344.

[19] Jarvis, P. G., 1976: The interpretation of the variations in leaf water potential and stomatal conductance found in canopies in the field, Phil. Trans. R. Soc. Lond. B. 273, 593-610..

[20] Kerzenmacher, T. E., 1995: Dynamisches Modell der Reaktion einer Fichte im Waldbestand auf den Wind, Diplomarbeit am Fachbereich Physik, Universität Göttingen, 67 S..

[21] Legg, B., Monteith, J., 1974: Heat and mass transfer within plant canopies in: De Vries, D. A., Afgan N. H. (ed.), Heat and mass transfer in the biosphere, part I: Transfer processes in plant environment, 167 - 186.

[22] Linke, F., Baur, F., 1970: Meteorologisches Taschenbuch, Neue Ausgabe, Band 2, Geest\&Portig, Leipzig.

[23] Laubach, J., 1991: Bestimmung der vertikalen turbulenten Wärme- und Wasserdampfstromdichten über einem Fichtenwald mit der Methode der Eddy - Korrelation, Diplomarbeit am Fachbereich Physik der Universität Göttingen.

[24] Löffler, J., 1994: Energiebilanz eines Fichtenbestandes: Eddykorrelationsmessungen der turbulenten Wärmeströme mit neuen Flußkorrekturverfahren, Diplomarbeit am Fachbereich Physik der Universität Göttingen. 
[25] Mießen, G., 1994: Modellierung des dreidimensionalen Transports von photosynthetisch aktiver Strahlung in einem Fichtenbestand im Solling, Diplomarbeit am Fachbereich Physik, Universität Göttingen, 63 S.

[26] Mix, W., Goldberg, V., Bernhardt, K.-H., 1994: Numerical experiments with different approaches for boundary layer modeling under large-area forest canopy conditions, Met. Zeits., N. F., 3, 187 - 192.

[27] Monteith, J. L., Unsworth M. H., 1973: Principles of Environmental Physics, New York, American Elsevier Publ. Co., 241 pp. .

[28] Ni, Wenge, 1996: A coupled transilience model for turbulent air flow within plant canopies and the planetary boundary layer, Agricul. For. Met., 86, 77 105.

[29] Oltchev, A., Constantin, J., Gravenhorst, G., Ibrom, A., 1997: A six-layer SVAT model for a simulation of water vapour and sensible heat fluxes in a spruce forest, J. Hydrol. Hydrom., 45, 1 - 2, 5 - 37.

[30] Raupach, M. R., Shaw, R. H., 1982: Averaging procedures for flow within vegetation canopies, Boundary-Layer Meteorol., 22, 79-90

[31] Raymond, W. H. and Stull, R. B., 1990: Application of Transilient Turbulence Theory to mesoscale numerical weather forecasting, Mon. Wea. Rev. 118, 2471 $-2499$.

[32] Riese, O., 1995: Turbulenter Austausch in und über einem Fichtenwald: Eddy - Korrelationsmessungen vertikaler Ströme latenter und fühlbarer Wärme, Diplomarbeit am Fachbereich Physik, Universität Göttingen, 110 S..

[33] Scheffer, F., Schachtschabel, P., 1984: Lehrbuch der Bodenkunde, Ferdinand Enke Verlag Stuttgart, 442 S..

[34] Schirmer, H. (wiss. Bearb.), 1987: Meyers Kleines Lexikon Meteorologie, herausgegeben und bearbeitet von Meyers Lexikonredaktion, Meyers Lexikonverlag Mannheim/Wien/Zürich, S. 496.

[35] Sellers, P. J., Sud, Y. C., Dalcher, A., 1986: A simple biosphere model (SIB) for use in general circulation models, J. Atmos. Sci., 43, 505 - 531.

[36] Stull, R. B. and Hasagawa, T., 1984: Transilient Turbulence Theory. Part II: Turbulent adjustment, J. Atmos. Sci., 41, 3368 - 3379.

[37] Stull, R. B., 1984: Transilient Turbulence Theory. Part I: The concept of eddy mixing across finite distances, J. Atmos. Sci., 41, 3351 - 3367.

[38] Stull, R. B., 1988: An introduction to boundary-layer meteorology, Dordrecht, Kluwer Academic Publishers, 665 pp.

[39] Stull, R. B., 1990:Nonlocal turbulent mixing: Measurement and parameterization of transilient matrices,Preprints Ninth Conference of Turbulence and Diffusion, April 30-May 3, 1990, Roskilde, Denmark. Amer. Meteor. Soc., $348-351$. 
[40] Stull, R. B., 1991: A comparison of parameterized vs. measured transilient mixing coefficients for a convective mixing layer, Boundary-Layer Meteorol. 55, $67-90$.

[41] Stull, R. B., 1993: Review of nonlocal mixing in turbulent atmospheres: Transilient Turbulence Theory, Boundary-Layer Meteorol., 62, 21 - 96.

[42] Stull, R. B. and Driedonks, A. G. M., 1987: Applications of the transilient turbulence parameterization to atmospheric boundary-layer simulations, Boundary-Layer Meteorol., 40, 209 - 239.

[43] Stull, R. B., Bartnicki, J., 1993: Mass-conserving turbulence and advection parameterizations for an eulerian air-pollution transport model, Technical Manual, Boundary Layer Research Team, Dept. of Atmospheric and Oceanic Sci., University of Wisconsin.

[44] Stull, R. B., 1996: Lecture notes for the Transilient Turbulence Workshop, held at the Institut of Bioclimatology, Göttingen University, Germany, May 1996.

[45] Witte, H., 1993: Temperaturen der Nadeloberflächen in einem Fichtenbestand im Solling - Feldmessungen und ihre Interpretation, Diplomarbeit am Fachbereich Physik, Universität Göttingen, 106 S..

[46] Yamada, T., 1982: A numerical model study of turbulent airflow in and above a forest canopy, J. Met. Soc. Japan, 60, 439 - 454.

[47] Zhang, Quing and Stull, R. B., 1992: Alternative nonlocal descriptions of boundary-layer evolution, J. Atmos. Sci., 49, 2267 - 2281. 


\section{Abbildungsverzeichnis}

1.1 Mittleres Profil der horizontalen Windgeschwindigkeit und für ausgewählte Höhen berechnete Diffusionskoeffizienten für Impuls in einem Fichtenbestand im Solling für den Juli 1994 . . . . .

1.2 Abhängigkeit der Differenz der Windgeschwindigkeit zwischen Kronen- und Stammraum in einem Fichtenbestand im Solling von der Windrichtung über dem Bestand (Halbstundenmittel 1997) . . . . . . . . . . . . . . . . . .

1.3 Abhängigkeit der Differenz der Windgeschwindigkeit zwischen Kronen- und Stammraum in einem Fichtenbestand im Solling von der thermischen Schichtung über dem Bestand (Halbstundenmittel 1997) . . . . . . . . . . . . . . . . . . .

1.4 Schematische Veränderung der Eigenschaftsprofile beim Durchund Überströmen eines Waldbestandes bei neutraler thermischer Schichtung am Beispiel der horizontalen Windgeschwindigkeit

3.1 Schematische Darstellung zweier Transilienzmatrizen für denselben atmosphärischen Prozeß für äquidistante und nichtäquidistante Einteilung der Modellschichten nach [31] . . . . . . . . . .

3.2 Schematische Struktur der konvektiven Grenzschicht nach Deardorff, dargestellt anhand der Vertikalprofile der potentiellen Temperatur $\Theta$ und des fühlbaren turbulenten Wärmeflusses $\overline{\Theta^{\prime} w^{\prime}}$. .

3.3 Auftreten unterschiedlicher Regimes der Turbulenz in der atmosphärischen Grenzschicht in Abhängigkeit von der vorherrschenden Art der Produktion turbulenter kinetischer Energie (TKE) (nach [38]) . . . . . . . . . . . . . . . . . . . . .

3.4 Anzahl der benötigten Iterationsschritte zur Berechnung der Transilienzmatrix aus der Mischungsmatrix als Funktion der gewünschten Genauigkeit für 2 spezielle atmosphärische Zustände (Erläuterung $i m$ Text $\ldots \ldots \ldots \ldots \ldots$. . . . . . . . . . . 39

3.5 Schritte bei der Berechnung der Transilienzmatrix . . . . . . . . 41

3.6 Beispiel einer Transilienzmatrix des verwendeten Grenzschichtmodells für einen Zeitschritt von $10 \mathrm{~min}$. . . . . . . . . . . . . 42 
3.7 Zuordnung von Transportrichtung und -geschwindigkeit zu verschiedenen Gebieten der Transilienzmatrix nach Ebert, Schumann und Stull $[11] \ldots \ldots$. . . . . . . . . . . . . . . .

4.1 Schematische Darstellung der geometrischen Parameter des idealisierten Bestandes in der Aufsicht: $\Delta_{\max }=$ maxim. Kronendurchmesser . . . . . . . . . . . . . . . . .

4.2 Schematische Darstellung der geometrischen Parameter eines Modellbaumes in der Seitenansicht . . . . . . . . . . . . . . . . . .

4.3 Vertikale Verteilung der Baumoberflächendichte und der cumulativen Baumoberfläche für den F1-Bestand im Solling - die grau unterlegten Bereiche kennzeichnen den Kronenraum . . . . . .

$5.150 \mathrm{~m}$-Meßturm des Institutes für Bioklimatologie im Fichtenbestand (F1-Fläche) im Solling . . . . . . . . . . . . . . . . . .

5.2 Schema des Grenzschichtmodells mit transilienter Turbulenz-

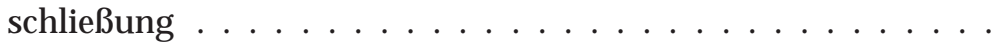

5.3 Schema der Verwendung der Meßwerte und der Analyse - und Prognosedaten des Wetterdienstmodells im atmosphärischen Teil des transilienten Grenzschichtmodells mit Waldbestand (Darstellung nicht maßstabsgerecht) . . . . . . . . . . . . .

5.4 Überblick über die wichtigsten physikalischen Prozesse, welche im transilienten Modell mit Baumbestand berücksichtigt werden.

6.1 Vergleich der gemessenen und berechneten kurzwelligen abwärtsgerichteten Strahlungsflußdichte in $2 \mathrm{~m}$ Höhe für den 20. und 21.06.1998 nach Meßdaten und Modellberechnungen . . . . . .

6.2 Berechnete und gemessene Bodentemperaturen für den 20.06.1998 86

6.3 Berechnete Profile der relativen Bodenfeuchte für den 20.06.1998

6.4 Berechnete turbul. Impulsflüsse und fühlbare Wärmeflüsse (oben) sowielatente Wärmeflüsse (unten) in und über dem Bestand für den 20.06.1998 bei stationärer Rechnung mit dem transilienten Modell . . . . . . . . . . . . . . . . .

6.5 Mit dem instationären K - Modell (lokale Turbulenzschließung oben) und mit dem transilienten Modell (transiliente Turbulenzschließung - unten) berechnete Profile der horizontalen Windgeschwindigkeit im Bestand für den 20.06.1998 im Vergleich mit den Meßwerten . . . . . . . . . . . . . . . . . .

6.6 Mit dem instationären K - Modell (lokale Turbulenzschließung - oben) und mit dem transilienten Modell (transiliente Turbulenzschließung - unten) berechnete Profile der Lufttemperatur im Bestand für den 20.06.1998 im Vergleich mit den Meßwerten 
6.7 Mit dem instationären K - Modell (lokale Turbulenzschließung oben) und mit dem transilienten Modell (transiliente Turbulenzschließung - unten) berechnete Profile der spezifischen Feuchte im Bestand für den 20.06.1998 im Vergleich mit den Meßwerten

6.8 Mit dem instationären K-Modell (lokale Turbulenzschließung links) und dem instationären transilienten Modell (transiliente Turbulenzschließung - rechts) berechnete turbulente Impulsflüsse für den $20.06 .1998 \mathrm{im}$ Bestand . . . . . . . . . . . . . . . .

6.9 Mit dem instationären K-Modell (lokale Turbulenzschließung links) und dem instationären transilienten Modell (transiliente Turbulenzschließung - rechts) berechnete turbulente Flüsse fühlbarer Wärme für den 20.06.1998 im Bestand . . . . . . . . . . . .

6.10 Mit dem instationären K-Modell (lokale Turbulenzschließung links) und dem instationären transilienten Modell (transiliente Turbulenzschließung - rechts) berechnete turbulente Flüsse latenter Wärme für den 20.06.1998 im Bestand . . . . . . . . . . .

6.11 Vergleich der mittels 2 Modellen (lokale Turbulenzschließung (K - Modell) und transiliente Turbulenzschließung (TTT - Modell)) berechneten und der mittels Eddy-Kovarianzverfahren bestimmten Impulsflüsse (oben) und fühlbaren Wärmeflüsse (unten) in $39 \mathrm{~m}$ Höhe für den $20.06 .1998 \ldots \ldots$. . . . . . . . . . . . . 101

6.12 Vergleich der mittels 2 Modellen (lokale Turbulenzschließung (K - Modell) und transiliente Turbulenzschließung (TTT - Modell)) berechneten und der mittels Eddy-Kovarianzverfahren bestimmten Flüsse latenter Wärme in $39 \mathrm{~m}$ Höhe über dem Bestand für den $20.06 .1998 \ldots \ldots$. . . . . . . . . . . . 102

6.13 Vergleich der mittels 2 Modellen (lokale Turbulenzschließung (K-Modell) und transiliente Turbulenzschließung (TTT-Modell)) berechneten Tagesgänge der Grenzschichthöhe für den 20.06.1998 103 


\section{Tabellenverzeichnis}

5.1 Überblick über die als Eingabeparameter des transilienten Modells verwendeten am $50 \mathrm{~m}$ - Meßturm gewonnenen Werte physikalischer Größen. . . . . . . . . . . . . . . . . . .

5.2 Im Modell verwendete pflanzenphysiologische Parameter im Bereich des Stamm- und Kronenraumes. . . . . . . . . . . . . . 62

5.3 Zur Modellierung der Bodentemperaturen benutzte Parameter. 68

5.4 Zur Modellierung der Bodenfeuchte benutzte Parameter. . . . . 70

5.5 Zur Modellierung der Energie- und Feuchteflüsse zwischen Vegetation bzw. Boden und Atmosphäre benutzte Parameter. . . .

5.6 Im Modell verwendete Parameter der transilienten Turbulenzschließung. Quelle: [18] . . . . . . . . . . . . . . . . . .

6.1 Vergleich der mit dem transilienten Modell in stationärer Rechnung berechneten turbulenten Flüsse von Impuls, fühlbarer und latenter Wärme in 39 m Höhe am 20.06.1998 mit den mittels Eddy - Kovarianz Verfahren bestimmten Flüssen . . . . . . . . . 


\section{Danksagung}

In erster Linie gilt mein Dank Prof. Dr. Gravenhorst für die Hilfe und die Unterstützung bei der Verwirklichung dieser Arbeit.

Herrn Prof. Dr. Eckelmann gilt mein Dank für die Ermöglichung und die Betreuung dieser Arbeit im Rahmen des Graduiertenkollegs „Strömungsinstabilitäten und Turbulenz".

Bei Herrn Prof. Dr. Ronneberger möchte ich mich für die Übernahme des Koreferates bedanken.

Frau Ziemann, Herrn Goldberg sowie den Herren Dr. Mix und Prof. Dr. Bernhardt (ehemals Meteorologisches Institut der Humboldt-Universität Berlin) gilt mein Dank für die Überlassung des K-Schließungsmodells für Vergleichsrechnungen.

Frau Dr. Inclán, Herr Dr. Constantin und Herr Dr. Oltchev haben mich mit vielen Diskussionen und Hinweisen unterstützt. Dafür gebührt ich ihnen mein Dank.

Beim Deutschen Wetterdienst möchte ich mich für die Überlassung der Analyseund Prognosedaten des Deutschlandmodells bedanken.

Ganz herzlich möchte ich mich bei allen Mitarbeitern des Institutes, inbesondere auch bei Frau Baumann für die immer tatkräftige Unterstützung bei der Arbeit danken. 


\section{Lebenslauf}

- geboren am 8. Juni 1970

- September 1977 bis Juli 1987: Besuch der Polytechnischen Oberschule in Randau

- September 1987 bis Juli 1989: Besuch der Erweiterten Polytechnischen Oberschule in Schönebeck/Elbe

- Juli 1989:

Erlangung der allgemeinen Hochschulreife

- September 1989 bis Februar 1995: Studium der Meteorologie an der Humboldt - Universität zu Berlin

- Februar 1995:

Diplomarbeit zum Thema: „Prognose der bodennahen Ozonkonzentration im Berliner Raum mittels Regressionsbeziehungen auf der Grundlage von Model-Output-Statistik"

- Oktober 1995 bis Oktober 1998: DFG-Stipendiat im Graduiertenkolleg der Universität Göttingen „Strömungsinstabilitäten und Turbulenz"

- $\quad$ seit Oktober 1998:

Mitarbeit am Institut für Bioklimatologie der Universität Göttingen . 